\title{
BEAMLINES AT SYNCHROTRON RADIATION FACILITIES: \\ THE LINK BETWEEN THE USER AND THE MACHINE
}

Erik D. Johnson, Steven L. Hulbert and Lonny IE. Berman

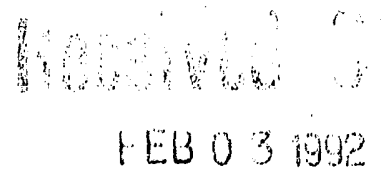

DECEMBER, 1991

Research Supported by the OFFICE OF BASIC ENERGY SCIENCES

\begin{abstract}
NATIONAL SYNCHROTKON LIGHT SOURCE BROOKHAVEN NATIONAL LABORATORY Associated Universitites, Inc.
\end{abstract}

\section{U.S. DEPARTMENT OF ENERGY} WASHINGTON, D.C.

Under Contract No. DE-AC02-76CH00016 


\section{DISCLAIMER}

This report was prepared as an account of work sponsored by an agency of the United State Government. Neither the United States Government nor any agency thereof, nor any of their employees, nor any of their contractors, sub-contractors, or their employees, makes any warranty, expressed or implied, or assumes any legal liability or responsibility for the accuracy, completeness, or usefulness of any information, apparatus, product, or process disclosed, or represents that its use would not infringe privately owned rights. Reference herein to any specific commercial product, process, or service by privately owned rights. Reference hereir to any specific commercial product, process, or service by trade name, trademark, manufacturer, or otherwise, does not neces arily constitute or imply its endorsement, recommendation, or favoring by the United States Government or any agency, contractor or subcontractor thereof. The views and opinions of authors expressed herein do not necessarily state or reflect those of the United States Government or any agency, contractor or subcontractor thercof. 
BEAMLINES AT SYNCHROTRON RADIATION FACILITIES:

THE LINK BETWEEN THE USER AND THE MACHINE

BNL- -47058

DE92 007186

Erik D. Johnson, Steven L. Hulbert and Lonny E. Berman

DECEMBER 1991

Research Supported by the

OFFICE OF BASIC ENERGY SCIENCES

\title{
NATIONAL SYNCHROTRON LIGHT SOURCE \\ BROOKHAVEN NATIONAL LABORATORY \\ Associated Universitites, Inc.
}

\author{
U.S. DEPARTMENT OF ENERGY \\ WASHINGTON, D.C. \\ Under Contract No. DE-AC02-76CH00016
}

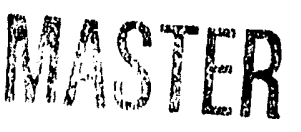


Beamlines at Synchrotron Radiation Facilities:

The Link Between the User and the Machine

Erik D. Johnson, Steven L. Hulbert and Lonny E. Berman

\author{
Brookhaven National Laboratory \\ National Synchrotron Light Source \\ Beamline Research and Development Group \\ Upton, New York 11973
}

\title{
TABLE OF CONTENTS
}

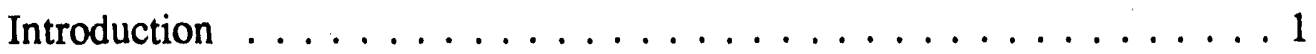

1.1 Beamline Perspective $\ldots \ldots \ldots \ldots \ldots$

1.2 Considerations for the Storage Ring .............. 3

2 The Source, Beamline Components, and Phase Space . . . . . . . . . . 4

$2.1 \quad$ Phase Space Representations . . . . . . . . . . . . . . . 6

2.2 Comparison of DuMond and M-K Spaces . . . . . . . . . 8

2.3 Slits in Phase Space .................... . . . . . . . . . . . . .

2.4 Source Phase Space . . . . . . . . . . . . . . 13

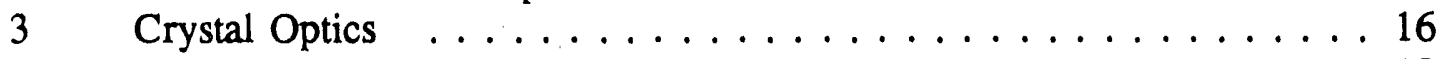

3.1 Crystal Monochromators ................. 18

3.1 .1 Anti-parallel configuration . . . . . . . . . . 18

3.1 .2 Parallel configuration . . . . . . . . . . . . 20

3.1.3 Comparison of Optical Arrangements .......... 26

3.2 Focussing Perfect Crystals . . . . . . . . . . . . . . 32

4 Geometric Optics Beamlines . . . . . . . . . . . . . . . . . . 34

4.1 Phase Space in Geometric Optics . . . . . . . . . . . . . . 36

4.2 Plane Grating Monochromators ... . . . . . . . . . . . 43

4.3 Spherical Grating Monochromators . . . . . . . . . . . . 44

4.4 Sensitivity to Storage Ring Parameters . . . . . . . . . . . 46

4.4.1 Current . . . . . . . . . . . . . . . 46

4.4.2 Vertical Beam Size and Position . . . . . . . . . . . . 46

4.4.3 Horizontal Beam Size and Position . . . . . . . . . . 47

4.4.4 Vertical Beam opening angle and angular position . . . . . 47

4.4.5 Forizontal Beam Opening Angle and Angular position . . 49

$5 \quad$ Concluding Remarks . . . . . . . . . . . . . . . . . . 5 50

$6 \quad$ References . . . . . . . . . . . . . . . . . . 51 


\section{Introduction}

At this point in time the literature is full of excellent review articles [1] which describe the operating principles of optical systems for utilizing the unique radiation provided by synchrotron storage rings. In general, the perspective provided by this body of work is that of the end user-experimenter cum optics designer. Nominal design specifications of the accelerator are usually assumed, and the impact of operation in a performance envelope which may represent either degraded or enhanced machine performance is seldom considered. In this article, we have attempted to remove ourselves from this (our own usual) perspective and look instead at the beamline as a transfer function to map from the machine to the users experiment.

We open first with an introduction to the perspective of the experimentalist, and some general considerations for the interaction of beamline hardware with the machine. We then discuss phase space representations of some common components of beamlines, and then treat some important classes of crystal and geometric optics in monochromators. We then close with a discussion of some of the common features of these optical systems, and the impact of the machine on user experiments.

\subsection{Beamline Perspective}

It is difficult to overstate the impact of synchrotron radiation research. From a little known obscure endeavor in the mid 1960's, it has advanced to a state of development such that essentially every area of scientific study has some level of involvement at an accelerator based research facility. One of the difficulties which has come with this success is the explosive growth of a new user community, not familiar with the vagaries of life at an accelerator, many of whom regard the synchrotron as a light bulb which is turned on upon demand, providing radiation which meets all the requirements of their particular experiment. While the state of accelerator technology advances daily the machine physicist cannot quite satisfy this naive picture, in part, because usually the radiation from the synchrotron is conditioned by a beamline before it reaches the users experiment. 


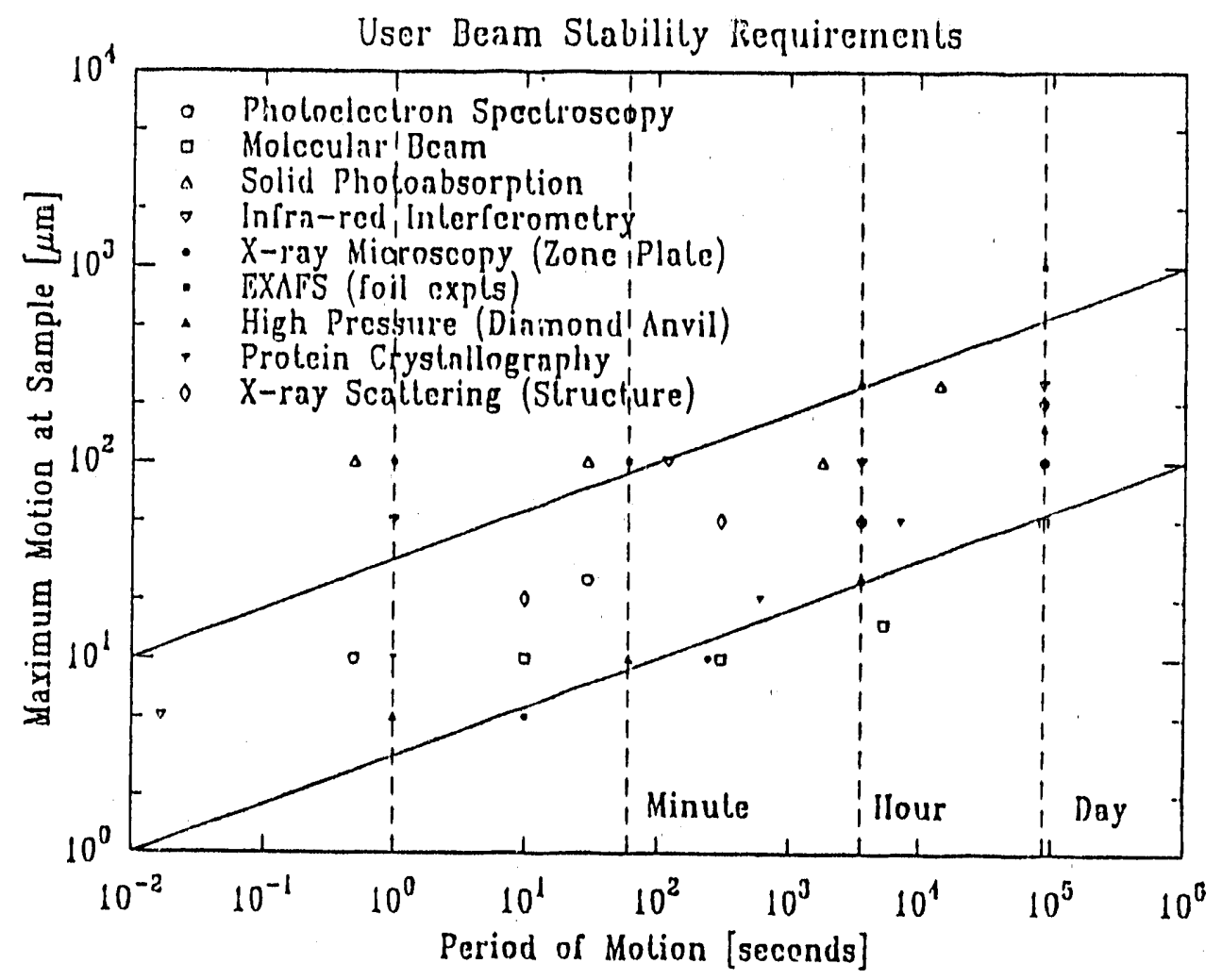

Fig. 1 Survey of user beam stability requirements at the NSLS for various experiments

Figure 1 provides a summary of the users perspective for several representative experiments with regard to stability. This informal survey crudely shows the state of experimental facilities provided by experimentalists in terms of their ability to compensate for beam motion at their sample as a function of the period or time scale of the beam motion. This picture provides no insight as to the source of the motion, that is to say "real" beam motion, or apparent motion due to the interaction of the machine with the beamline. This latter category includes (but is not limited to) effects such as beamline component heating, vibration induced by water flow, motion from changes in beamline components due to temperature variations and many others. Some of these problems can enter into the experiments in very subtle ways, as variations in beam position, wavelength, and bandwidth. To gain a qualitative understanding of the impact of beamlines on experiments, one must first consider the experimental requirements, and the demands they make on the source by way of the beamline. 


\subsection{Considerations for the Storage Ring}

Nearly all of the experiments performed at a synchrotron radiation facility utilize some type of beam relay or collection optics, and most require some kind of monochromator to select a specific wavelength and bandwidth of radiation for their experiment. For the purposes of this discussion, the combination of these elements along with their environment comprise a beamline. The range of wavelengths which are required by the experiment have a significant effect on the beamline design and configuration and its possible impact on the machine. For convenience we will break experiments into three wavelength ranges, vacuum ultra-violet to infra-red $(\lambda>100$ $\mathrm{nm}$, energies less than $13 \mathrm{eV})$, soft $\mathrm{x}$-rays $(100 \mathrm{~nm}<\lambda<.6 \mathrm{~nm}$, energies from $13 \mathrm{eV}$ to $2 \mathrm{keV})$, and $\mathrm{x}$-rays $(\lambda>.6 \mathrm{~nm}$, energies above $2 \mathrm{keV})$.

From the standpoint of machine security, the low energy beamlines present the biggest problem since they must share machine vacuum due to the lack of suitable window materials for the transmission of the radiation. What this means is that the beamline vacuum hardware must be interlocked with the ring, and to the greatest extent possible, separated by differential pumping, acoustic delays, and fast acting valves to minimize the impact of beamline vacuum failures on the machine operation.

For hard $\mathrm{x}$-ray beamlines, the beamline vacuum is typically separated from the machine vacuum by a water cooled beryllium window roughly 0.01 " thick. Properly supported, such a window can withstand atmospheric pressure, so the quality of the beamline vacuum need not be on par with that required for the storage ring. In fact, at some facilities, the beamline pipe is purged with helium at atmospheric pressure.

The wavelength groupings also appear in terms of the optical design of the beamlines. IR and VUV radiation can be reflected at or near normal incidence by a variety of materials but, as the wavelength decreases, the reflection efficiency drops precipitously. In these cases the optics are arranged at grazing incidence angles, often 2 degrees or less, to achieve reflection efficiencies of 10's of percent in the soft $\mathrm{x}$-ray range. Because of the small angles involved, optics of this type are quite difficult to fabricate, and therefore expensive, since they become physically large in order to insure that some reasonable fraction of the photon beam can be collected. This in turn places significant constraints on the maximum excursion of the beam in the machine before the intensity (and possibly wavelength) variations become too large to be acceptable at the experiment. An additional requirement of these optics is the precision with which they must be figured and finished, again due to the small angles involved: specified slope errors the order of a few arcseconds have become common in high resolution soft $\mathrm{x}$-ray monochromators. This type of beamline also places significant demands on the floor of the building to provide a stable bench for 
the optical components which may be 5 or more meters apart yet require positional stability the order of 10 microns (in the case of slits for example). For these reasons, grazing incidence optics beamlines may well place the tightest constraints on the operation of the storage ring.

For hard $x$-ray beamlines the technology becomes rather different, since perfect crystal optics based on silicon and germanium come into their own at energies above $2 \mathrm{keV}$. The precision in the dispersive element is provided by the crystal structure and Bragg reflectivities, even at large angles of incidence, can be well over $50 \%$. The crystals can be quite small, and depending upon their arrangement, all of the important elements can be mounted on one fairly small assembly. The characteristics of crystal optics are in fact so attractive that numerous attempts have been made to extend the techniques to longer wavelengths.

One of the difficulties thus far has been the lack of robust crystals with the requisite interplanar spacings. In the past, mica, beryl, and a variety of synthetic organic crystals have been used, but all seem to be fairly sensitive to beam damage. Recently work has been directed to the fabrication of synthetic multilayer structures to replace the crystals, although the degree of regularity required in the interfaces and interlayer spacings is thus far at or beyond the state of the art. Another promising avenue for development is in the growth of large lattice constant inorganic crystals. The successful growth of large crystals of $\mathrm{YB}_{66}$ [2] has recently been reported, and holds great promise for extending crystal optics down to $1 \mathrm{keV}$.

\subsection{The Source, Beamline Components, and Phase Space}

Both bending magnet and insertion device based sources are readily available at synchrotron radiation facilities, but for this article, we couch the discussion in terms of dipole sources only. The treatment of the beamline optics is however general, and need only be adjusted for the actual parameters of the source under consideration. For our present discussion we shall only briefly review dipole sources wherein the photons emerging from the synchrotron are emitted tangentially by electrons as they are deflected in the plane of their closed orbit, or horizontal plane; this plane of emergence has a narrow vertical thickness that depends on the angular divergences of the photons from a given optical axis or tangent, and on the distance propagated from the origin of this tangent (see Fig. 2). The photons can be described as a collection of points in a three-dimensional displacement-divergencewavelength space (henceforth known as Matsushita-Kaminaga, or M-K, space, after the two who originally formulated it), each point specifying a particular combination of displacement, angular divergence, and distance from the origin of the optical axis. This type of description has appeared previously for crystal optics systems [3] from 
which much of present work is derived, and in several excellent reviews [4-0]. in this work we extend the general features of the approach to include geometric optical systems.

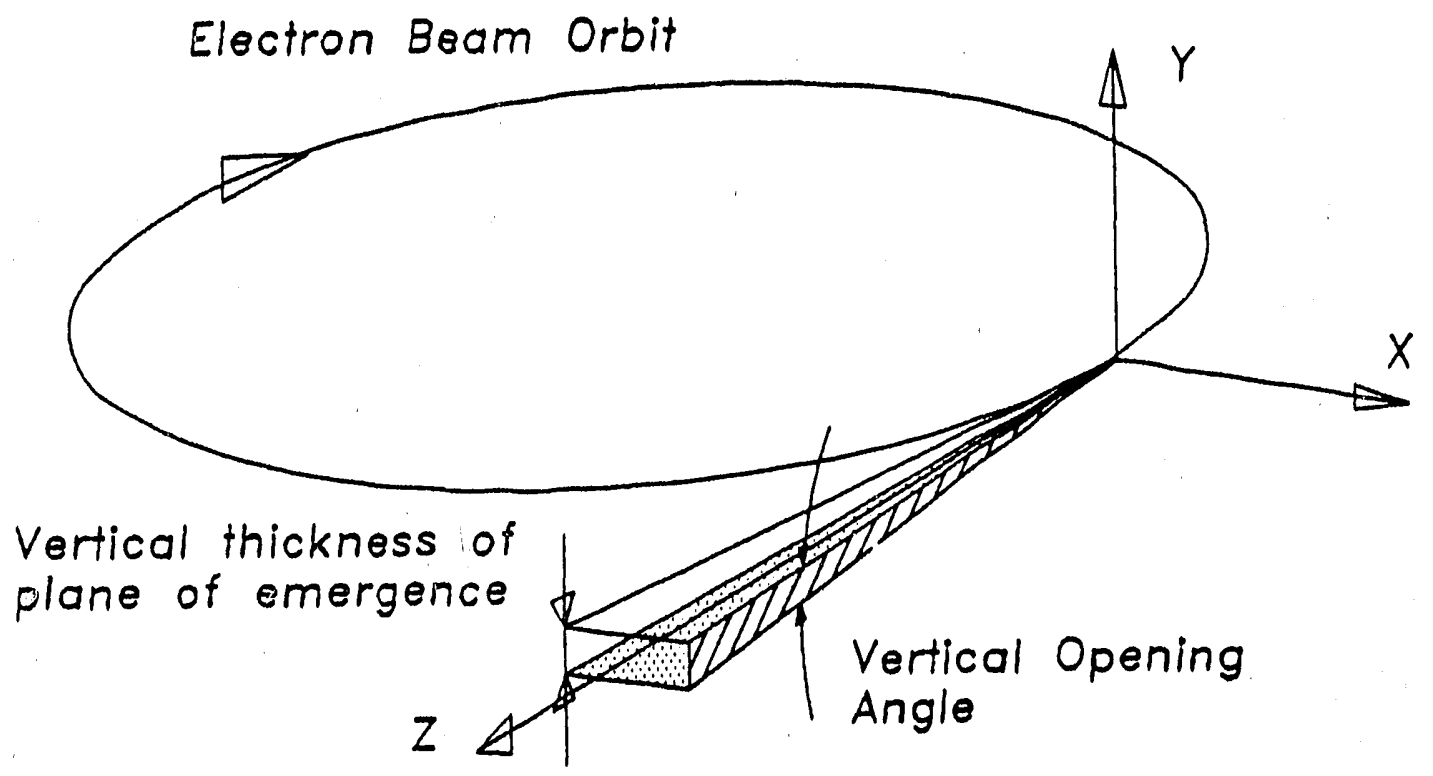

Fig. 2 Photon beam emerging from a synchrotron source.

Optical elements, such as mirrors, gratings, diffracting crystals and slits, that pass finite ranges of divergence and displacement in the vertical plane (for given wavelengths) may be introduced along the optical axis. The "windows of emergence" characterizing such elements can also be presented by volumes in M-K space. By observing how volumes characterizing the photon source and optical elements are oriented with respect to one another, the $\mathrm{M}-\mathrm{K}$ coordinates of photons that would be requisite for their passage by a particular system can be determined. Hence, by judiciously choosing an appropriate combination of elements, and by knowing the emittance properties of the source, one can literally select a bandwidth, or spread of wavelengths about a central one, to extract for use in experiments. The size of the M-K "volume of emergence" will also determine the intensity within the bandwidth extracted. This bandwidth is constant along the horizontal plane -- and experimental apparatus that is oriented in this plane will therefore "catch" mure intensity than one that isn't. 


\subsection{Phase Space Representations}

From here on, only motion along one axis (the z-axis) is cnnsidered, and only the $y-p_{y}$ phase plane (motion in the $y-z$ cartesian plane) is considered. We wish to trace the movement of points in the $y-p_{y}$ plane with changing $z$, or changing time $t$, equivalent to tracing the propagation of particles in the vertical $y-z$ plane. Calling $z$ the optical axis, we define $y$ as the vertical displacement from this axis, and $y$ ' as the vertical angular divergence from this axis. (see Fig. 2). For small divergences $y^{\prime}$ is proportional to $p_{y}$ :

$$
y^{\prime} \approx \frac{d y}{d z}=\frac{d y / d t}{d z / d t}=\frac{p_{y}}{p_{z}}, p_{z}=\text { constant }
$$

The particles propagate at a uniform (or near-uniform, owing to variations in y') velocity along the $z$-axis. Hence, we may study their motion by considering areas in a plane with $y$ - and $y^{\prime}$-axes.

A device is something that either generates a beam, or is placed in its path and acts upon it ("system", as we have been using it, can be taken to be the environment composed of a device and a beam). Assume that the device is placed at a particular $z$. The acceptance $A$ of the device is defined as $1 / \pi$ times the area in the $y-y^{\prime}$ phase plane which contains the $\left(y, y^{\prime}\right)$ coordinates that are accepted for transmission by the device. Particles whose coordinates do not fall within this area are rejected. The emittance $\epsilon$ of the device is defined as $1 / \pi$ times the area in the phase plane which contains the $\left(y, y^{\prime}\right)$ coordinates of particles that can emerge from the device. The acceptance and emittance of a device do not have to coincide in shape -- they must in magnitude, provided that a Hamiltonian can be described for particles around or within the device (in which case Liouville's Theorem applies).

Typical devices for $\mathrm{x}$-ray work are the synchrotron source (characterized by an emittance only), slits, crystals, and mirrors. When two devices are placed adjacent to each other, the particles transmitted are the ones who ie coordinates fall within the area of intersection of the emittance of the first device and the acceptance of the second one (see Fig. 3).

Free space of any length has essentially infinite acceptance and emittance. Consider free space of length $d$. The transfer of a beam particle across this length is described by the ray matrix $\left[\begin{array}{ll}1 & d \\ 0 & 1\end{array}\right]$ where, $\left[\begin{array}{c}y_{\text {emerging }}^{y^{\prime}} \\ y_{\text {emerging }}\end{array}\right]=\left[\begin{array}{ll}1 & d \\ 0 & 1\end{array}\right]\left[\begin{array}{c}y_{\text {in }} \\ y^{\prime} \\ \text { in }\end{array}\right]$. (Any device's transmission properties can be described by a phase plane transformation matrix.) In the $y-y$ ' phase plane, the area that describes a beam will become 
"skewed" along the y-axis as free space is traversed (see Fig. 4). Hence, a laigti $\mathrm{d}$ of free space is a device that will accept any beam, and will allow it to emerge with a different phase plane shape, but the same area.
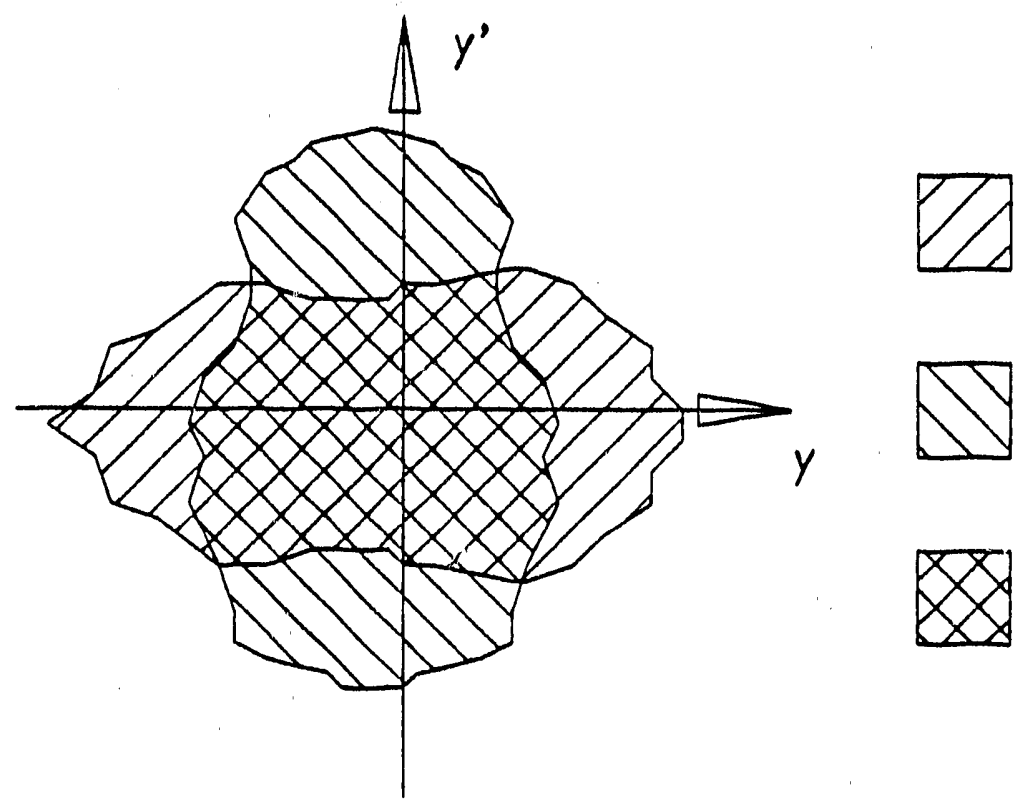

Emittance of

First Device

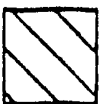

Acceptance of

Second Device

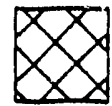

Transmitted Beam

Fig. 3 Representation of adjacent devices in the $y-y$ ' phase plane.

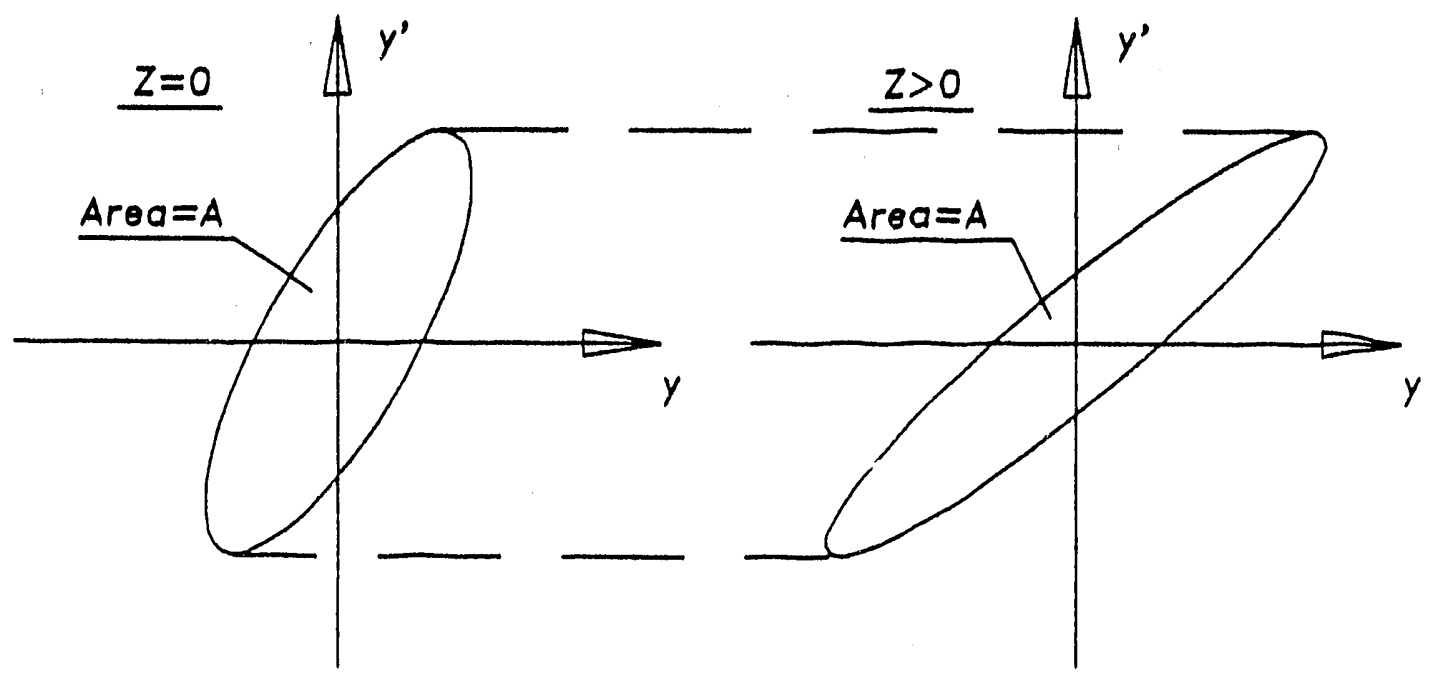

Fig. 4 Phase plane "skewing" of a beam traversing free space 


\subsection{Comparison of DuMond and M-K Phase Spaces}
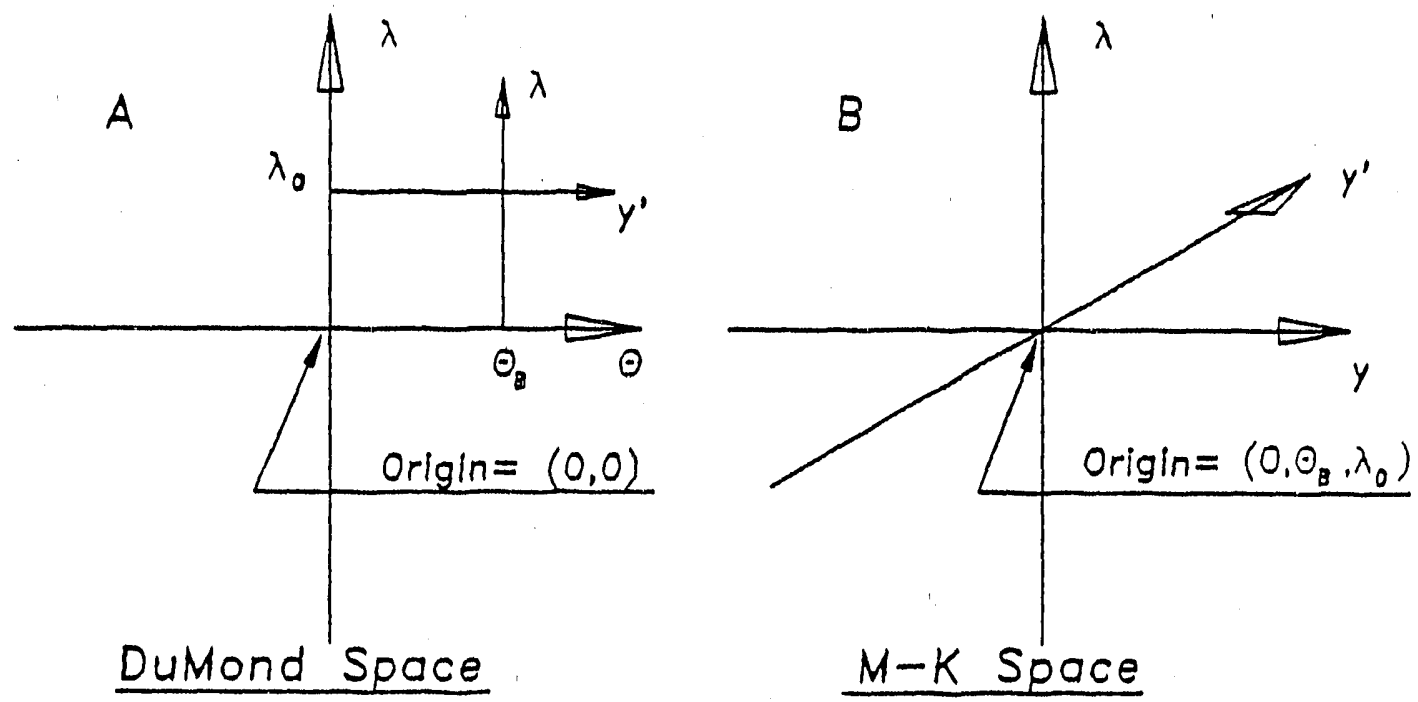

Fig. 5 Comparison of (a) DuMond and (b) M-K spaces.

When considering photons emerging from a synchrotron source, the preceding discussion concerning phase space carries the assumption that all parameters (e.g., wavelength) except for the phase coordinates and time are fixed. To incorporate the wavelength distribution of photons emitted from a source, a wavelength $(\lambda)$ axis may be added orthogonal to the $y-y$ ' phase plane. DuMlord [7] treated the subject of $x$ ray intensities and angular widths for systems consisting of a generator and diffracting crystals by analyzing areas of acceptance and emittance in a $\theta-\lambda$ plane, analogous to analyzing areas in the $y-y^{\prime}$ plane, where $\theta$ denotes the angle between the x-ray beam direction (optical axis) and the diffracting lattice planes of a crystal (see Fig. 5a). Matsushita and Kaminaga [6] described the performance of optical devices for a synchrotron source by analyzing volumes of acceptance and emittance in a $y-y^{\prime}-\lambda$ space. The $y-y^{\prime}-\lambda$, or $M-K$, space is realized as a combination of the $y-y^{\prime}$ and $\theta-\lambda$ spaces by setting the origin, or zero, of $y^{\prime}$ equivalent to some nominal angle $\theta_{B}$, and by setting the origin, or zero, of $\lambda$ equivalent to some nominal wavelength $\lambda_{0}$ (see Fig. 5b). y' thus denotes small excursions in $\theta$ from $\theta_{B}$, and $\lambda$ denotes small excursions in $\lambda$ from $\lambda_{0}$.

Before discussing optical devices in $\mathrm{M}-\mathrm{K}$ space, treatments in phase and in DuMond space are undertaken. 


\subsection{Slits in Phase Space}

Again when considering photons emerging trom a synchrotron source, a narrow angular spread is desired when a narrow energy spread of photons is desired (it will be shown later why this is so). The angular spread of a beam in the $y-z$ plane is the maximum width of the beam's corresponding phase are in the $y-y$ ' plane. The simplest devices used to narrow the angular spread of a beam are collimating slits.

Consider a beam blanketing a siit of width $2 \mathrm{~d}$, positioned symmetrically about the z-axis (see Fig. 6a). This slit serves to accept photons within $\pm d$ of the $z$-axis; any angular divergence $y$ ' within these confines is accepted. Hence, one slit places limits on the transmitted beam along the $y$-axis in the phase plane; no limits are placued along the y'-axis. The transmitted beam corresponds to the shaded phase area shown in Fig. 6b. The phase plane transformation matrices corresponding to a slit are $\left[\begin{array}{ll}1 & 0 \\ 0 & 1\end{array}\right]$ for $-d \leq y \leq d$, and $\left[\begin{array}{ll}0 & 0 \\ 0 & 0\end{array}\right]$ for $|y|>d$.
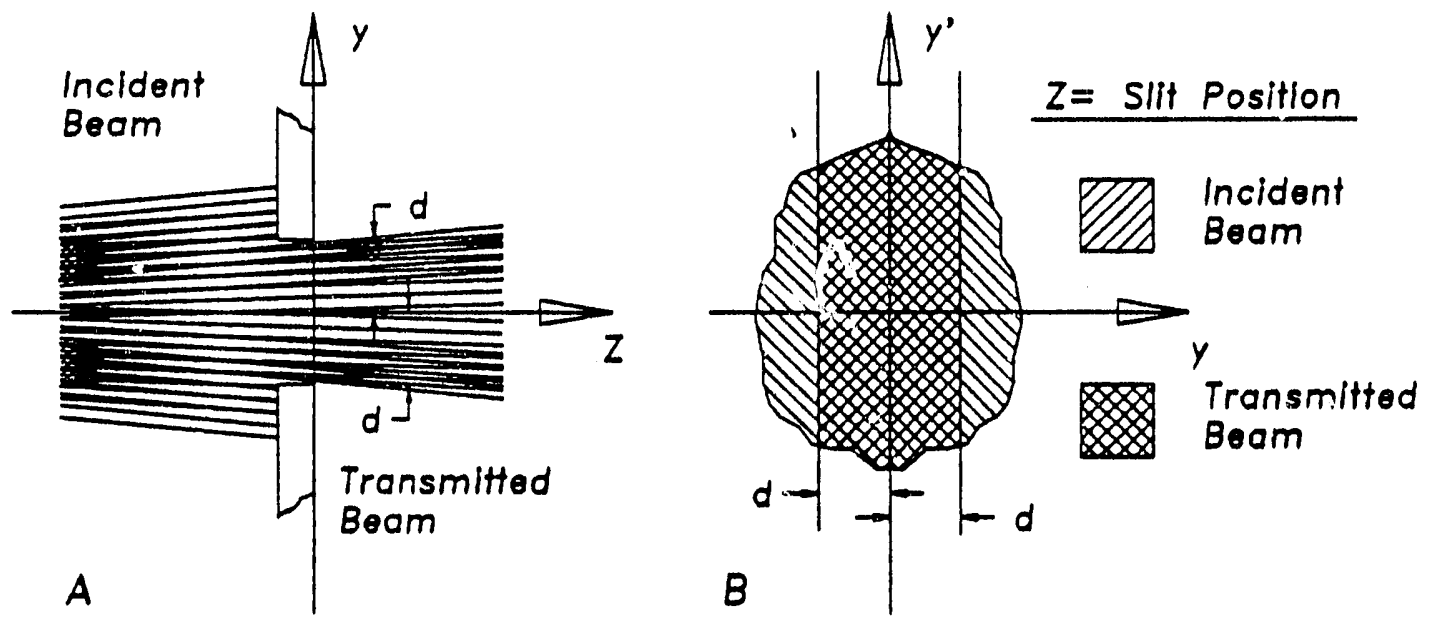

Fig. 6 (a) Single vertically-collimating slit of height $2 \pi$.

(b) Phase plane representation.

Now consider two slits separated by a distance $l$ (see Fig. 7a). The width of the first slit is $2 d_{1}$, and that of the second is $2 d_{2}$. Both are symmetric about the $\mathrm{z}$-axis. Assume the beam blankets the first slit. Fig. $7 \mathrm{~b}$ shows its evolution in the $y-y^{\prime}$ phase plane. 

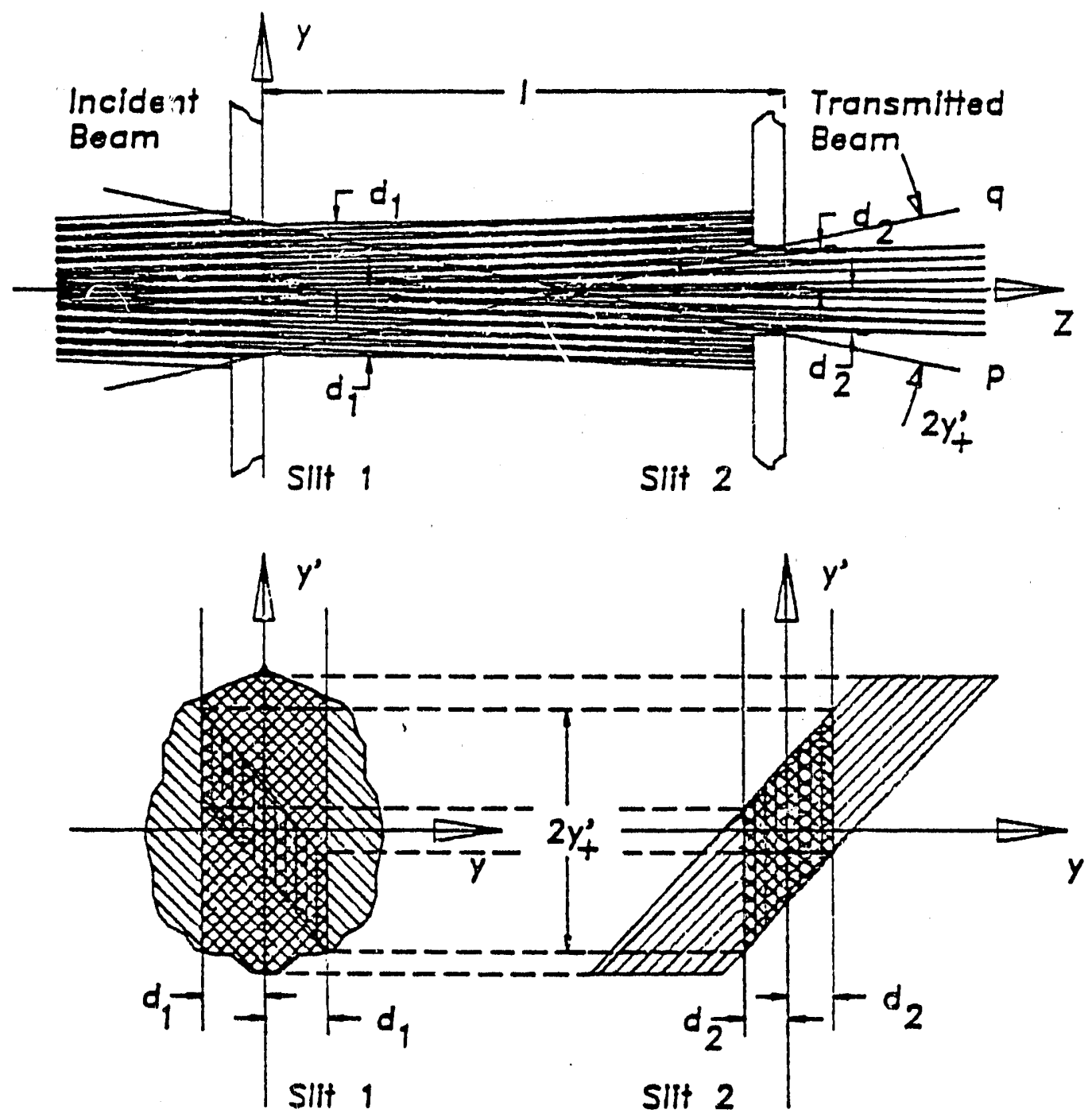

(A) DIA Incident

(B)

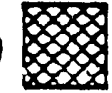

Transmitted Beam at Silt 1

(c)

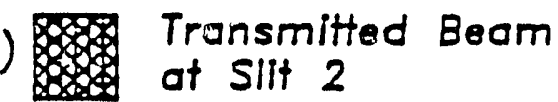

Fig. 7 Two vertically-collimating slits of respective heights $2 d_{1}$ and $2 d_{2}$ and separation $\ell$ (b) Phase plane representation 
The angular spread of the beam emerging from the second slit is $2 y_{i}{ }^{\prime} \ldots, i, \ldots$ confined to a "cone" delimited by rays $p$ and $q$. In the phase plane, the bsain area accepted at the first slit is the area B. This area is transformed acress the length $l$ thro'tgh the free space matrix $\left[\begin{array}{ll}1 & 1 \\ 0 & 1\end{array}\right]$, its size being conserved via Liouville's Theorem. Only the portion of $B$ within $\pm d_{2}$ of the $z$-axis is passed by the second slit. Taken as a system, the two slits accept the parallelogram $C$ at the first slit, and emit $\mathrm{C}$ at the second one. In traversing the length $l$, the shape of $\mathrm{C}$ changes, but its area is conserved and is found to be

$$
\begin{aligned}
2 d 1\left(\tan ^{-1}\left(d_{1}+\frac{d_{2}}{I}\right)-\tan ^{-1}\left(\frac{d_{1}-d_{2}}{I}\right)\right) & =2 d_{2}\left(\tan ^{-1}\left(\frac{d_{1}+d_{2}}{1}\right)+\tan ^{-1}\left(\frac{d_{1}-d_{2}}{1}\right)\right) \\
& =\frac{4 d_{1} d_{2}}{I}, d_{1}, d_{2}<1 .
\end{aligned}
$$

The angular spread of the emerging beam is

$$
2 y_{t}^{\prime}=2 \tan ^{-1}\left(\frac{d_{1}+d_{2}}{1}\right) \approx \frac{2\left(d_{1}+d_{2}\right)}{1}, d_{1}, d_{2}<1 \text {. }
$$

If $\mathrm{d}_{1}=\mathrm{d}_{2}=\mathrm{d}$, then $2 \mathrm{y}_{\mathrm{t}}{ }^{\prime} \simeq 4 \mathrm{~d} / l=2 \times$ (slit width)/ (slit separation). The fraction of incident photons passed is the ratio of the area of $\mathrm{C}$ to the area of $\mathrm{A}$ (assuming the phase area point density is uniform), or $4 \mathrm{~d}_{1} \mathrm{~d}_{2} /\left(\ell \times[\text { area }]_{\mathrm{A}}\right)$. The transformatic $n$ matrices for this system are obtained by multiplying those for the second slit, free space of length $l$, and first slit; they are $\left[\begin{array}{ll}1 & 1 \\ 0 & 1\end{array}\right]$ for $\left(y, y^{\prime}\right)$ falling within the confines of $B$ at the first slit, and $\left[\begin{array}{ll}0 & 0 \\ 0 & 0\end{array}\right]$ otherwise.

Typical phase areas from synchrotron sources vary; lets take a iectangular $[a r e a]_{A}=.1 m m-m r a d$, with a width along the $y^{\prime}$-axis of $.1 \mathrm{mrad}$. Suppose we ask for a divergence $2 \mathrm{y}_{\mathrm{t}}{ }^{\prime}=.01 \mathrm{mrad}$. If we introduced two slits of width $2 \mathrm{~d}=.1 \mathrm{~mm}$ (one-tenth the incident beam width), we would have to separate them by

$$
I=\frac{4 d}{2 y_{t}^{\prime}}=\frac{.2 \mathrm{~mm}}{.01 \mathrm{mrad}}=\frac{.2 \times 10^{-3} \mathrm{~m}}{.01 \times 10^{-3} \mathrm{rad}}=20 \mathrm{~m} \text {. }
$$

The fraction of incident photons that will pass is

$$
\frac{(2 d)^{2}}{I \times[a r e a]_{A}}=\frac{(0.1 \mathrm{~mm})^{2}}{20 \mathrm{~m} \times 0.1 \mathrm{~mm}-\mathrm{mrad}}=\frac{\left(0.1 \times 10^{-3} \mathrm{~m}\right)^{2}}{20 \mathrm{~m} \times 0.1 \times 10^{-6} \mathrm{~m}-\mathrm{rad}}=0.58 \text {. }
$$

so $99.5 \%$ of out incident beam is lost. 

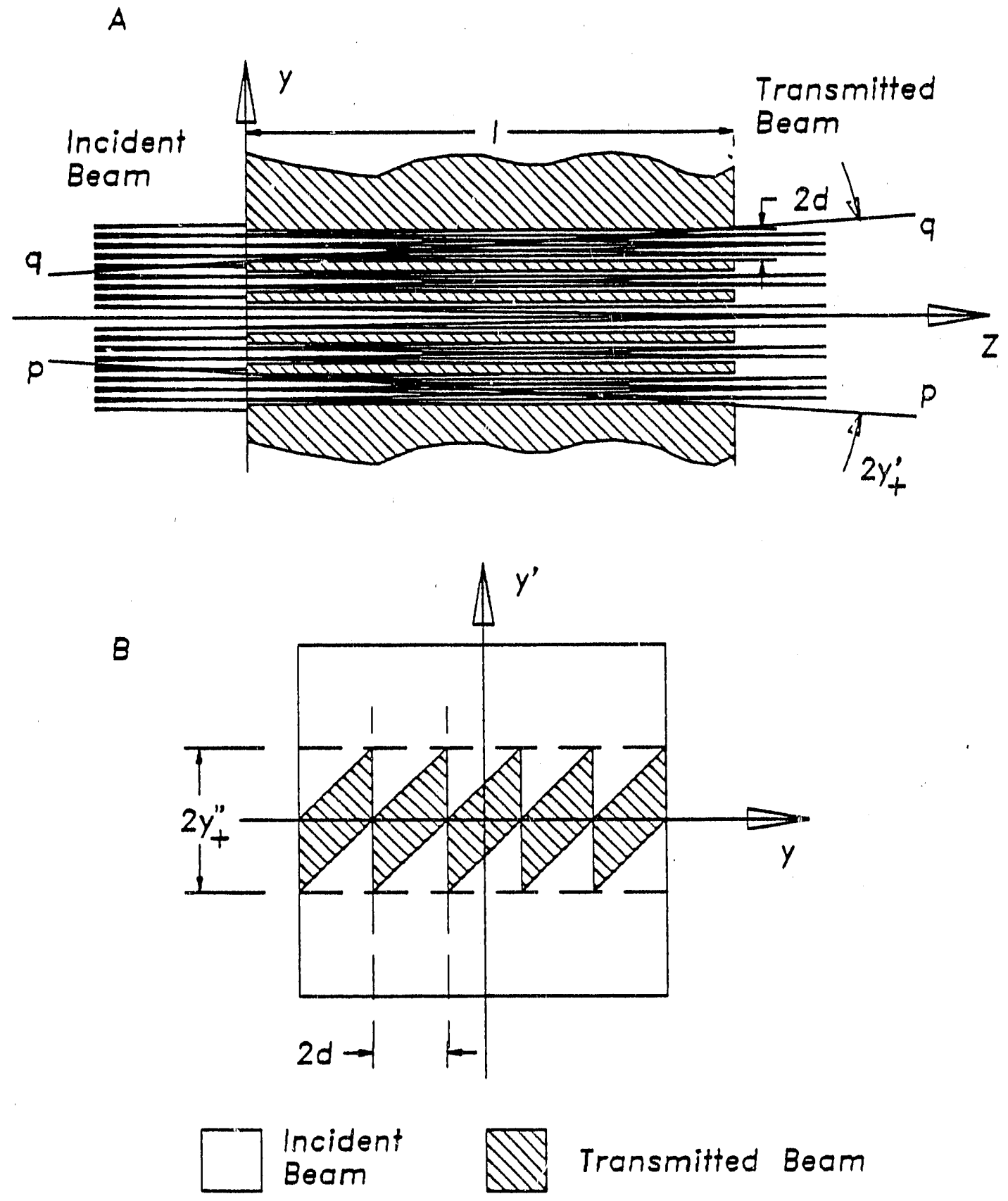

Fig. 8 (a) Stack of vertically Soller slits, each of height $2 d$ and length $\ell$. (b) Phase plane representation 
To pass more beam while preserving a desired angular spread, Soller slitis may be used. A set of such slits is a set of planes of thin absorbing material uniformly spaced $2 \mathrm{~d}$ apart from each other and of length $l$ along the $z$-axis (assume $d<<l$ ). If placed symmetrically about the $z$-axis, with enough planes stacked so as to "cover" the incident beam, the entire beam width (along the y-axis) may be passed with the angular spread narrowed to $2 y_{t}{ }^{\prime}=4 d / l$ (see Fig. 8a). In the $y-y^{\prime}$ phase plane, the situation is analogous to stringing side-by-side as many double slit (width $=2 \mathrm{~d}$, separation $=l$ ) parallelograms as the number of plane spacings filled by the beam (see Fig. $8 b$ ). Denoting this number by $N$, the fraction of incident photons passed is approximately $\mathrm{Nx}(2 \mathrm{~d})^{2} /\left(l \times[\text { area }]_{\mathrm{A}}\right)$. Using the same rectangular area $\mathrm{A}$ for the incident beam, with $2 \mathrm{~d}=.1 \mathrm{~mm}$ and the desired spread $=2 \mathrm{y}_{\mathrm{t}}{ }^{\prime}=.01 \mathrm{mrad}$., we need $\ell=4 d / 2 y_{t}^{\prime}=20 \mathrm{~mm}$. The entire beam width is passed for ten spacings $(\mathrm{N}$ $=10$ ), in which case $10 \times .5 \%=5 \%$ of the incident photon beam survives.

\subsection{The Source Phase Space}

An electron beam in a storage ring at a given $z$ may be described by the $y-y^{\prime}$ phase ellipse (see Fig. 9)

$$
\gamma y^{2} \cdot 2 \alpha y y^{\prime}+\beta y^{\prime 2}=\text { beam emittance } \epsilon,
$$

where $\gamma=\left(\alpha^{2}+1\right) / \beta$ and $\alpha=-\beta / 2=-\mathrm{d} \beta / 2 \mathrm{dz}[8] . \quad \alpha, \beta$, and $\epsilon$ are parameters characterizing the beam. (Much of the upcoming notations concerning areas in the $y-y$ ' phase plane and volumes in $M-K$ space are taken from Refs. 5 and 6. )

The electrons emit, tangent to their orbit, photons with an energy spread depending on the energy of the electrons and the radius of their orbit, with characteristic vertical angular spread $\sigma$ that depend on the photon energy ( $\sigma$ is smaller for higher photon energies). For a particular wavelength, each electron in the beam emits photons with a Gaussian angular spread in the $y-z$ plane; $\sigma$ is the "cone angle" within which one Gaussian standard deviation, or $-67 \%$, of the emitted photons fall, $2 \sigma$ is the cone angle within which two Gaussian standard deviations fall, etc. For a one- $\sigma$ contour of photons at a particular wavelength, a photon phase ellipse (see Fig. 10) may be constructed from the electron phase ellipse depicted in Fig. 9 through a convolution of the angular distributions of the electrons in the beam pipe and the photons emitted by each electron [8]. The equation for this new ellipse is

$$
\left(\gamma+\frac{\sigma^{2}}{E}\right) y^{2}+2 \alpha y y^{\prime}+\beta y^{\prime 2}=\epsilon+\beta \sigma^{2}
$$




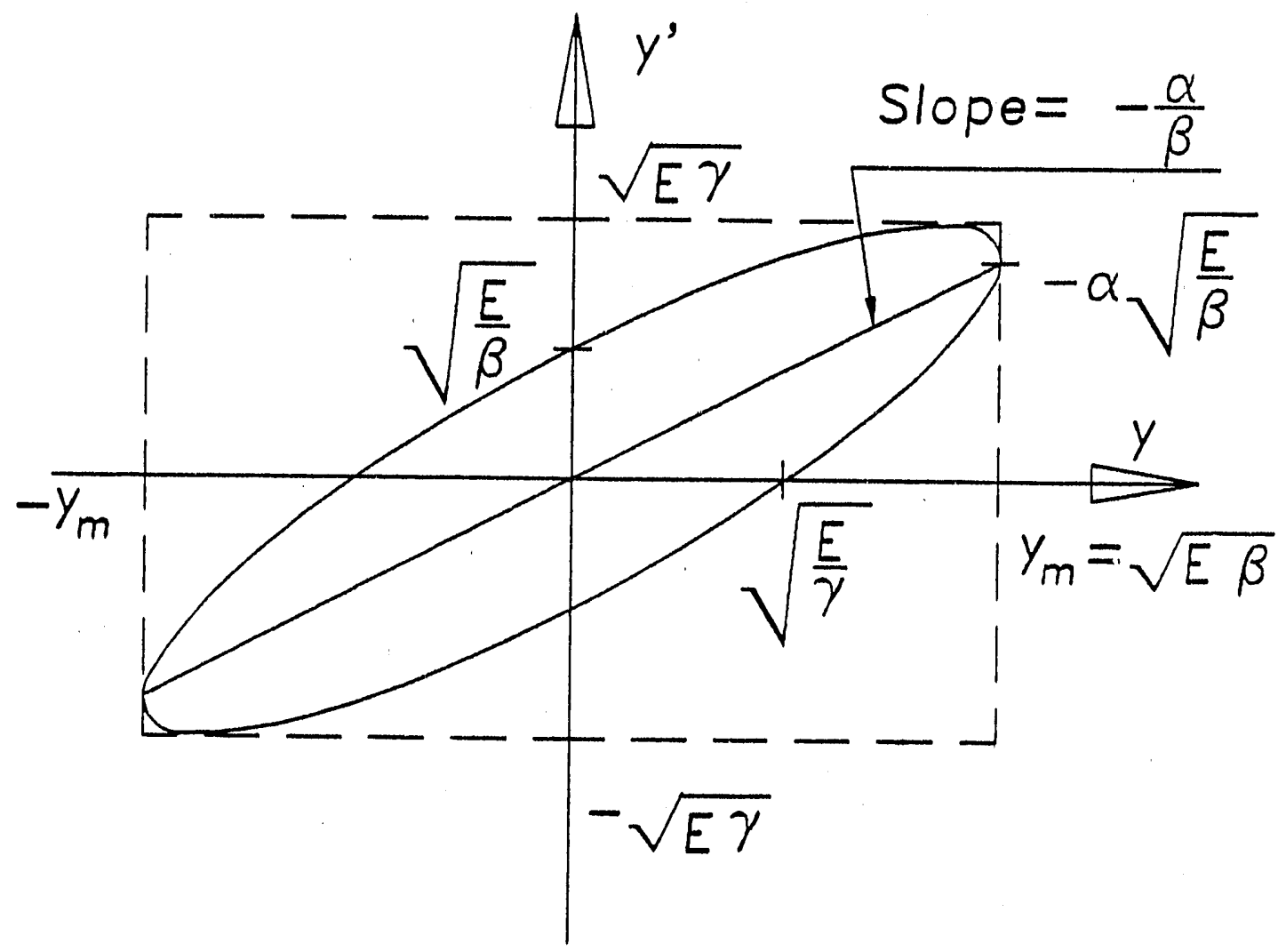

Figure 9 Storage ring electron beam phase ellipse.

Notice that the electron and photon ellipses have common spatial widths $2(\beta E)^{1 / 2}$, and common diameters, but that the photon beam has a larger divergence spread than the electron beam. This is the result of the addition in quadrature of the cone angle $\sigma$; the common diameter indicates that each electron emits photons symmetrical in angle with respect to the direction of its motion [5].

A photon beam that has propagated a distance $z$ from the storage ring can be described by a photon phase ellipse with area $S_{s y}=\pi\left(\epsilon\left[\epsilon+\beta \sigma^{2}\right]\right)^{1 / 2}$, maximum angular divergence $\Delta y^{\prime}{ }_{m}=2\left(\epsilon \gamma+\sigma^{2}\right)^{1 / 2}$, maximum spatial width $\Delta \mathrm{y}_{\mathrm{m}}=2\left(\left[\epsilon \gamma+\sigma^{2}\right] \mathrm{z}^{2}-2 \alpha \epsilon z+\beta \epsilon\right)^{1 / 2}=2\left(\left[\mathrm{y}_{\mathrm{m}}{ }^{\prime} \mathrm{z}\right]^{2}-2 \alpha \epsilon z+\beta \epsilon\right)^{1 / 2}$, and constant source size (as seen by an observer looking along the z-axis) $\Delta y_{0}{ }^{\prime}=2 S_{s y} / \pi y^{\prime}{ }_{m}$. 


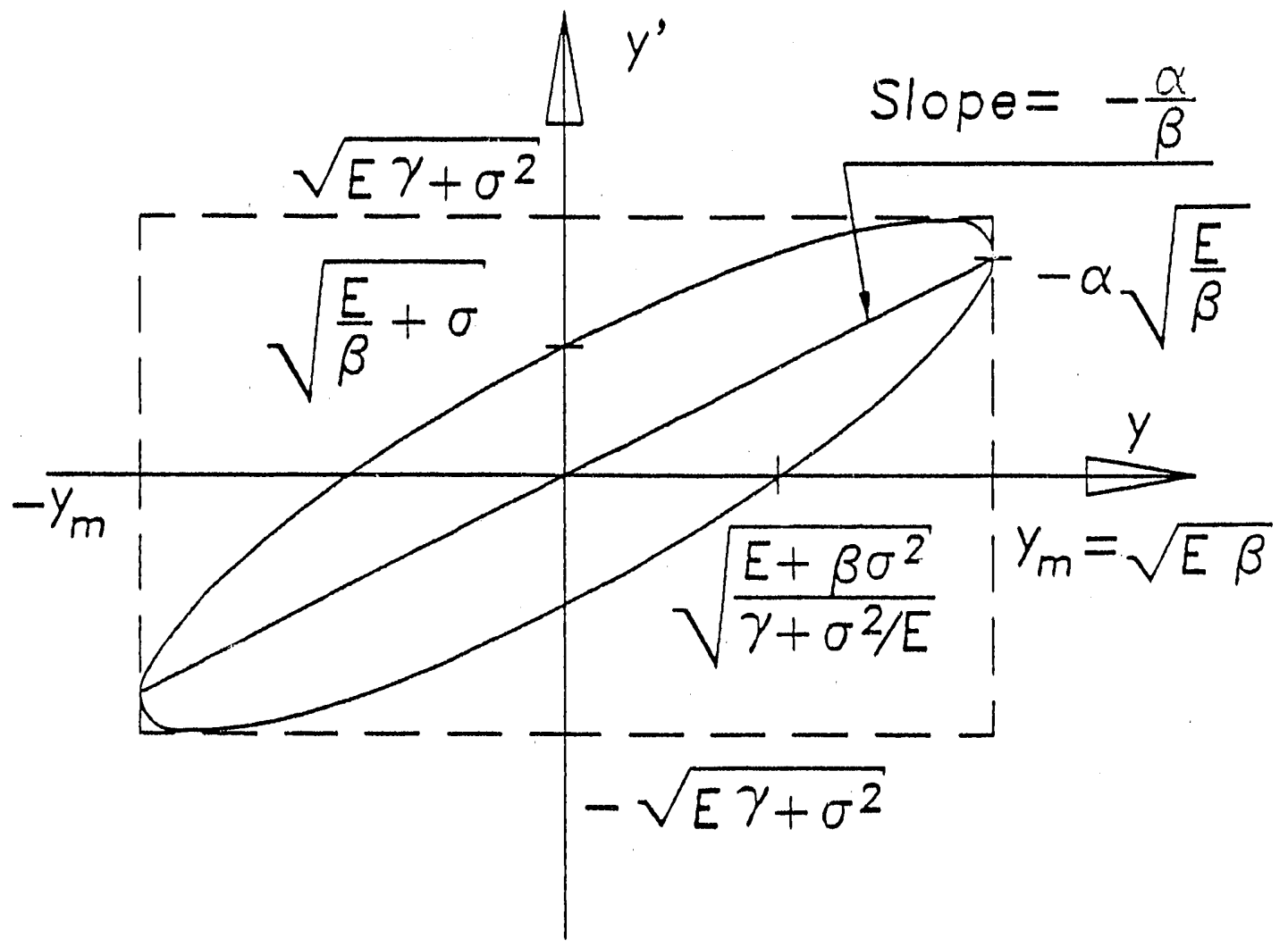

Fig. 10 Photon beam phase ellipse.

These photon ellipses are described for one particular wavelength. Ellipses will differ for different wavelengths, due to the variation in the cone angle $\sigma$ :

$1 \sigma(\operatorname{mrad})=\frac{0.645}{1.957 \times E(\text { GeV }) \times\left(\frac{\lambda_{c}}{\lambda}\right)^{0.549}}, \lambda_{c}>\lambda$,

$$
\text { where } \begin{aligned}
\dot{\lambda}(\dot{A}) & =\text { electron beam energy } \\
\lambda_{c}(\dot{A}) & =\text { critical wavelength }=\frac{5.59 R(\mathrm{~m})}{E^{3}(\mathrm{GeV})} \\
\text { and } R & =\text { radius of electron beam orbit. }
\end{aligned}
$$

The critical wavelength $\lambda_{c}$ has the property that one-half of the emitted radiation intensity from the electron beam is spread over wavelengths below $\lambda_{c}$, and one-half over wavelengths above $\lambda_{c}$. For a given $\lambda$, the area of the corresponding $y-y^{\prime}$ photon phase ellipse is proportional to the total number of photons $\mathrm{N}$ emitted integrated over all vertical divergences $y^{\prime}$ per unit wavelength (energy) spread, 
provided that the spread in $\lambda$ is extremely small (since $N$ is a sharply vitriti,li, function of $\lambda$ except near $\lambda_{c}$ ). In accordance with the previous assumption of a large number of electrons filling the beam pipe with random displacements and phases, the $\mathrm{N}$ photons are represented by $\mathrm{y}^{-} \mathrm{y}^{\prime}$ phase plane points uniformly distributed throughout the electron phase ellipse. The photon number is given by

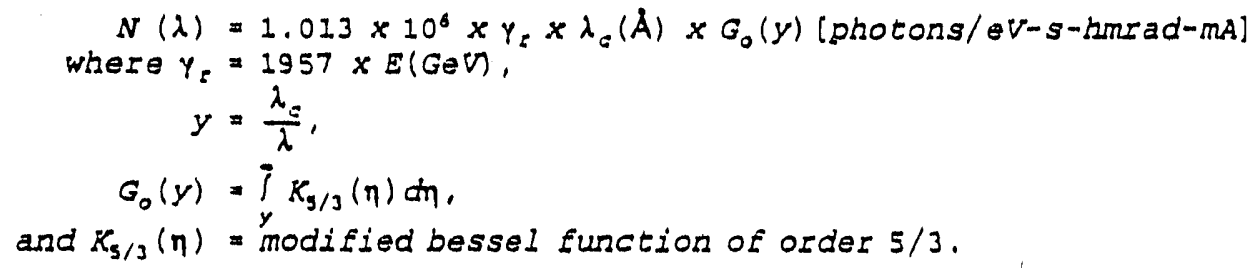

See Ref. 9 for details.

\subsection{Perfect Crystal Optics}

The intensity of photons of wavelength $\lambda_{0}$ will suffer no loss upon reflection from a perfect crystal if Bragg's law $n \lambda_{0}=2 d \sin \theta_{B}$ is satisfied, where $n$ is an integer, $\theta_{\mathrm{B}}$ is the angle between the incident photons and particular parallel lattice planes of the crystal (these are the diffracting planes), and $d$ is the spacing of these planes. One sees that photons of wavelength $\lambda_{0}$ incident on these planes at $\theta_{B}$ will be elastically reflected for $n \lambda_{0} \leq 2 d$. There is a finite angular width $w$ about $\theta_{B}$ over which perfect reflection occurs; for $\theta$ 's falling outside of $\theta_{B} \pm w / 2$, the photons are absorbed by the crystal, unless Biagg's law is satisfied for a different order of reflection $n$, or reflection from a different set of lattice planes of the crystal. $w_{0}$, the angular or Darwin width accepted for perfect reflection, is given by

$$
w_{0}=\frac{2}{\sin 2 \theta_{B}} \frac{e^{2} \lambda_{0}^{2}}{\pi m v c^{2}}\left|F_{h k 1}\right| \frac{1}{\sqrt{|b|}} P_{1}
$$

where $\mathrm{m}$ and $\mathrm{e}$ are the electronic mass and charge, respectively; $\mathrm{v}$ is the unit cell volume of the crystal; $c$ is the velocity of light; $\left|F_{h k l}\right|$ is the magnitude of the structure factor of the ( $\mathrm{hkl}$ ) lattice planes, and is a decreasing function of $n / 2 d$, with $\mathrm{d}=\mathrm{a}_{\mathrm{o}} /\left(\mathrm{h}^{2}+\mathrm{k}^{2}+\mathrm{l}^{2}\right)^{1 / 2}$, where $\mathrm{a}_{0}$ is the lattice parameter of the crystal;

$b=\sin \left(\phi+\theta_{B}\right) / \sin \left(\phi-\theta_{B}\right)$, where $\phi$ is the angle between the diffracting lattice planes and the crystal surface of incidence; and $\mathrm{P}$ is the polarization factor given by $\mathrm{P}=1$ for $\sigma(\mathrm{TE})$ polarization and $\mathrm{P}=\left|\cos 2 \theta_{\mathrm{B}}\right|$ for $\pi(\mathrm{TM})$ polarization [5]. Radiation of $\lambda_{o}$ falling within $\pm w_{o} / 2$ of $\theta_{B}$ will emerge within $\pm w_{h} / 2$ of $\theta_{B}$, where $w_{h}=w_{0}|b|$. 
For $\phi=0,|\mathrm{~b}|=1$, and the diffraction is symmetric; for $|\mathrm{b}| \neq 1$, the diffraction is asymmetric. For experiments using fixed wavelengths, an asymmetric diffraction is advantageous, for a large angular spread $w_{0}$ emerging from the source can be narrowed to a smaller spread $w_{h}$, improving resolution (to be shown shortly). For studies employing varying wavelengths, a problem that arises for asymmetric diffractions is the variation of $|\mathrm{b}|$ with wavelength. From here on, $|\mathrm{b}|=1$ and $P=1$ (we'll assume that the synchrotron radiation is completely polarized in the orbit $[x-z]$ plane, and that the $y-z$ plane will be our plane of incidence).

DuMond originally incorporated Darwin widths in plots of $\lambda$ vs. $\theta$ [7]. For reflection from a single crystal in first order $(n=1)$, Bragg's law can be plotted in the $\theta-\lambda$ plane. A band of width $w(\theta)$ about this relation can be superimposed (see Fig. 11). A photon of wavelength $\lambda$ and angle of incidence $\theta$ is reflected from the crystal if $(\theta, \lambda)$ falls within the shaded band in Fig. 11, and is absorbed if not.

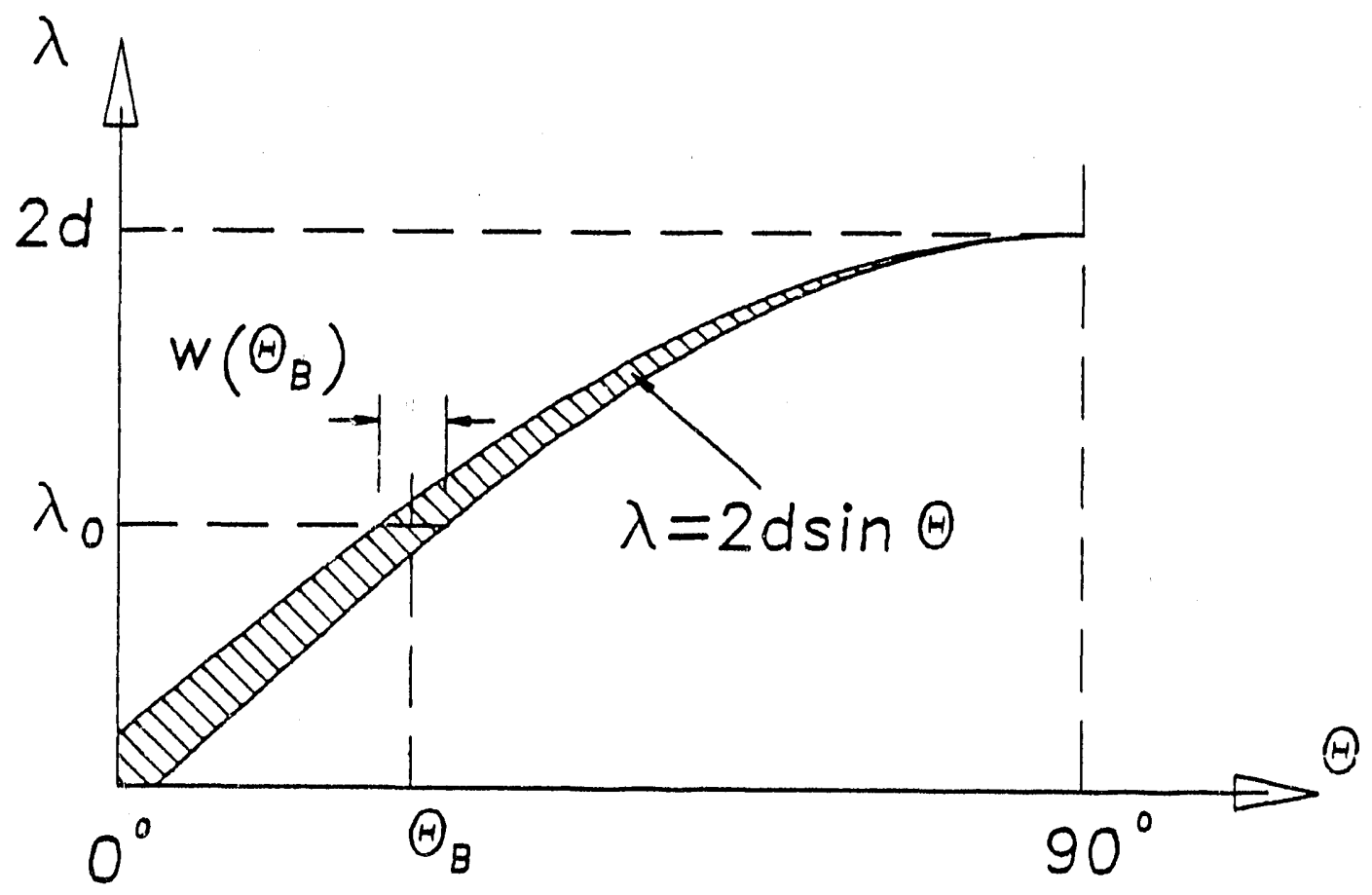

Fig. 11 DuMond band for one crystal. 
Differentiating Bragg's law

$$
d \lambda=\frac{2 d}{n} \cos \theta_{B} d \theta=\lambda_{0} \cot \theta_{B} d \theta
$$

where $\mathrm{d} \lambda<\lambda_{0}, \mathrm{~d} \theta<\theta_{\mathrm{B}}$. Photons incident on the crystal with an angular spread $w$ about $\theta_{B}$ will be reflected if iheir wavelength is approximately $\lambda_{0}$; since $w<<\theta_{B}$ (typical Darwin widths are on the order of seconds, while typical Bragg angles used are at least a few degrees), their wavelength spread is $d \lambda=\lambda_{0} w \cot \Theta_{B}$ about $\lambda_{0} . d \lambda$ is an indication of the wavelength resolution obtained if a white (polychromatic) beam is incident on the crystal with all photons incident at $\Theta_{B}$; this resolution is proportional to the Darwin width for $\theta_{B}$.

\subsection{Crystal Monochromators}

\subsubsection{Anti-parallel Crystals}

DuMond used these plots to analyze the transmission properties of multiplecrystal spectrometers, composed of crystals arranged so that $x$-rays of wavelength $\lambda_{0}$ are incident on each crystal at their corresponding $\theta_{\mathrm{B}}$. Consider the double-crystal spectrometer in the anti-parallel mode (see Fig. 12).

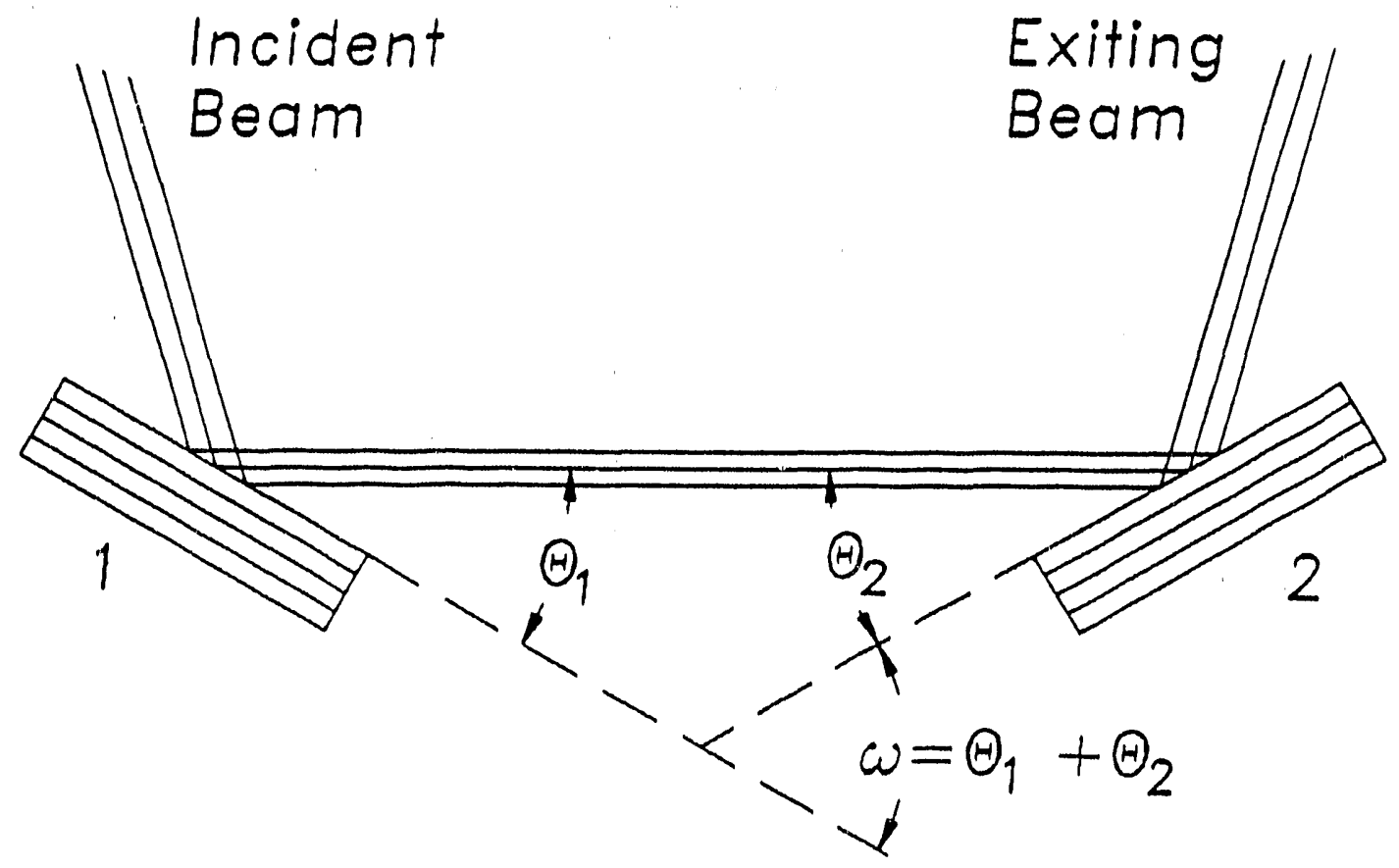

Fig. 12 Anti-parallel double crystal spectrometer. 


$$
\frac{d \theta}{d \lambda}=\frac{d \theta_{1}+d \theta_{2}}{d \lambda}=\frac{1}{d \lambda}\left(\frac{d \lambda}{\lambda_{0} \cot \theta_{1}}+\frac{d \lambda}{\lambda_{0} \cot \theta_{2}}\right)=\frac{\tan \theta_{1}+\tan \theta_{2}}{\lambda_{0}} .
$$

for $\theta_{1}=\theta_{2}=\theta_{B}, d \theta=2 w$, and $d \lambda=\lambda_{0} w \cot \theta_{B}$. For one crystal, the resolution obtained is effectively limited by the source angular divergence, while for the antiparallel double-crystal spectrometer, the crystal's Darwin width is the limiting factor. This spectrometer has been useful for applications where very high wavelength resolution was required [11].

\subsubsection{Parallel Crystals}

Figs. 14 and 15 show the double-crystal spectrometer in the parallel arrange:nent and its component single-crystal DuMond bands, respectively.

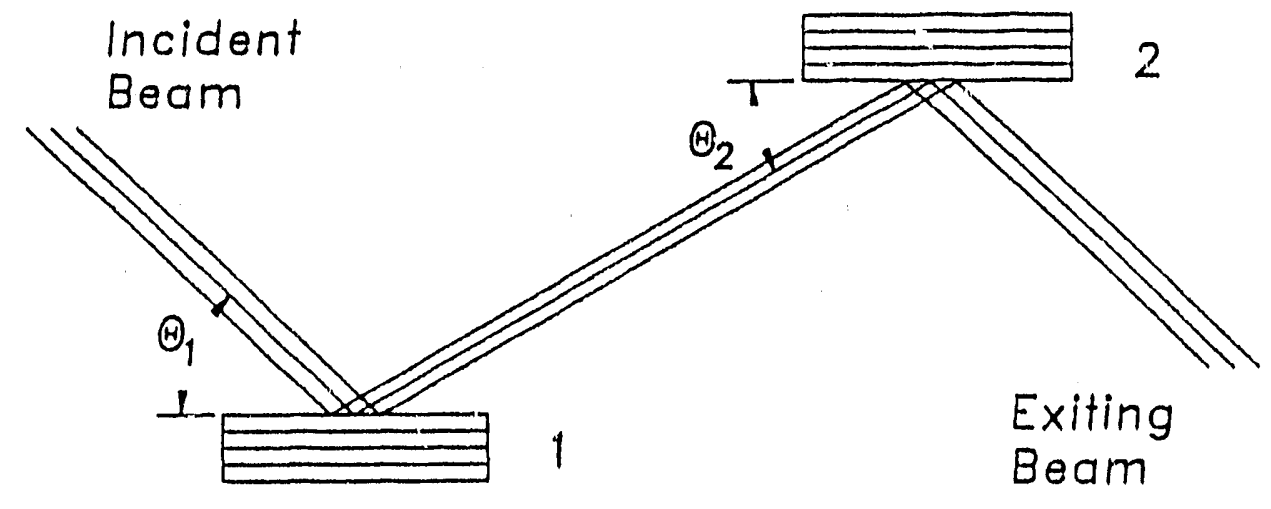

Fig. 14 Parallel double-crystal spectrometer.

These bands are oriented in the same sense along the $\theta$-axis, for increasing $\theta_{1}$ increases $\theta_{2}$, and vice versa (if the crystals are held fixed while the incident beam direction is varied). If $\theta_{1}$ is varied with respect to $\theta_{2}$, the bands will slide along the $\theta$-axis with respect to each other. If $\theta_{1}=\theta_{2}$, the bands will be superimposed.

If a large spread of wavelengths is incident upon these crystals over a large angular spread, this arrangement is seen to offer the same result as the single-crystal arrangement regarding the narrowing of the spreads. This is evident even if the overlap of the DuMond bands isn't complete, since almost any type of overlap will, like each DuMond band, cover a wide range of angles and wavelengths [see Fig. 16]. 
The two crystals can be represented as intersecting single-crystal DuMiulid bands oriented in the opposite sense alon's the $\theta$-axis (see Fig. 13), since an increase in $\theta_{1}$ will cause a decrease in $\theta_{2}$, and vice versa (if the crystals are held fixed while the incident beam direction is varied).

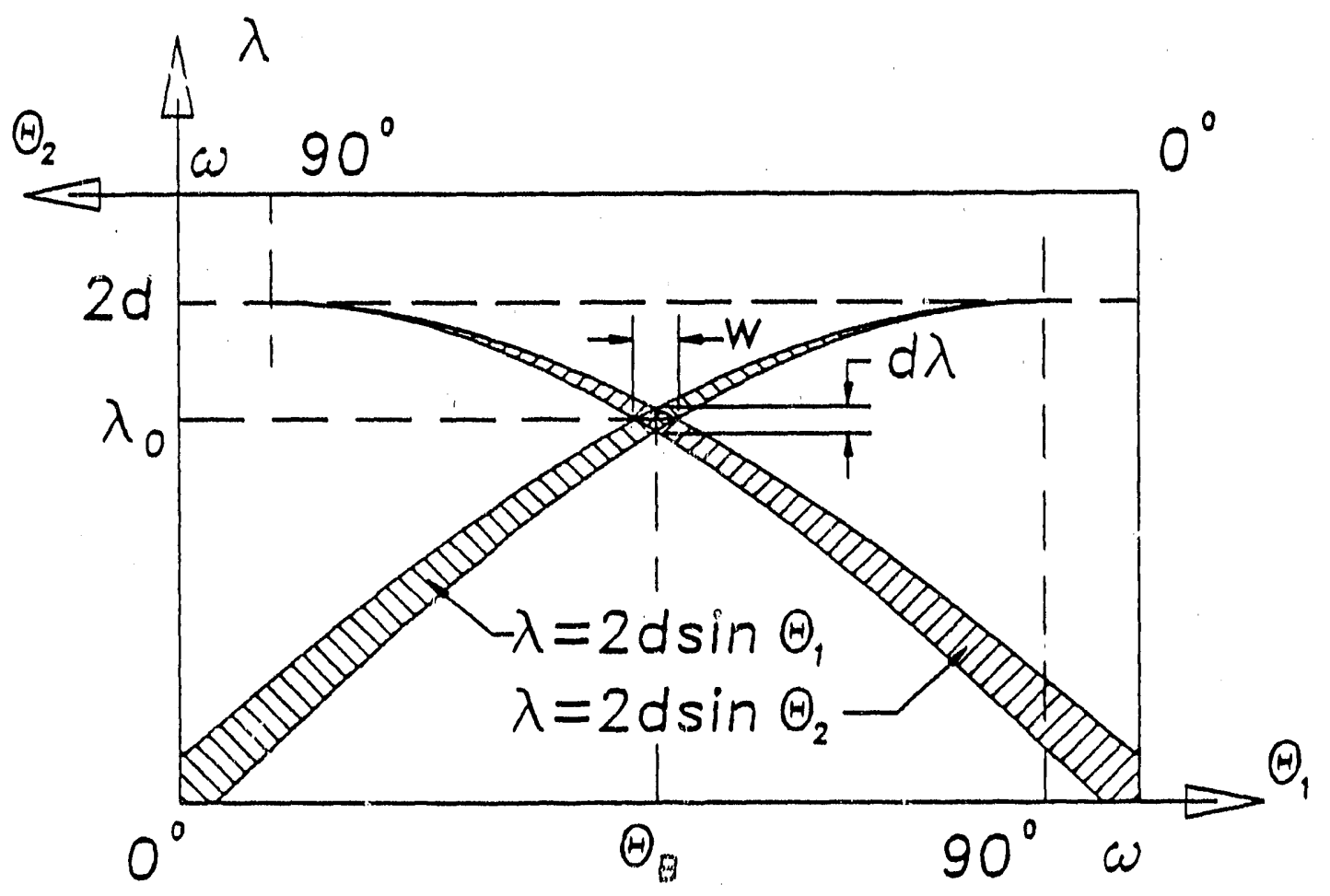

Fig. 13 DuMond representation for anti-parallel double-crystal spectrometer.

If $\theta_{1}=\theta_{2}=\theta_{B}$, only those photons falling within $\pm w / 2$ of $\theta_{B}$ for each crystal can be reflected. The ones that do emerge possess wavelengths such that their $(\theta, \lambda)$ coordinates fall within the quasi-quadrilateral of intersection (which is rhombus-like) of the two DuMond bands. This area of intersection is proportional to the intensity of photons that are passed by this system (this area is small enough so that its point density is uniform).

Suppose a large spread of wavelengths $\lambda$ is incident upon these crystals over a large angular spread about $\theta_{\mathrm{B}}$. If only one crystal is present, large angular and wavelength spreads are passed if the various wavelengths are incident at their corresponding Bragg angles to within $\pm w\left(\Theta_{B}\right) / 2$. For the anti-parallel double-crystal spectrometer, though, only the angular spread $w$ is passed (the width of the area of intersection of the DuMond bands), giving a wavelength spread about $\lambda_{0}$ of $d \lambda=\lambda_{0}$ $w \cot \theta_{B}$ (the height of the area of intersection). In general, for the crystals oriented at $\theta_{1}$, and $\theta_{2}$, the resulting dispersion $d \theta / d \lambda$ obtained is [10] 
The effective dispersion is

$$
\frac{d \theta}{d \lambda}=\frac{\tan \theta_{1}-\tan \theta_{2}}{\lambda_{0}}
$$

If $\theta_{1}=\theta_{2}$, then $d \theta / d \lambda=0$.

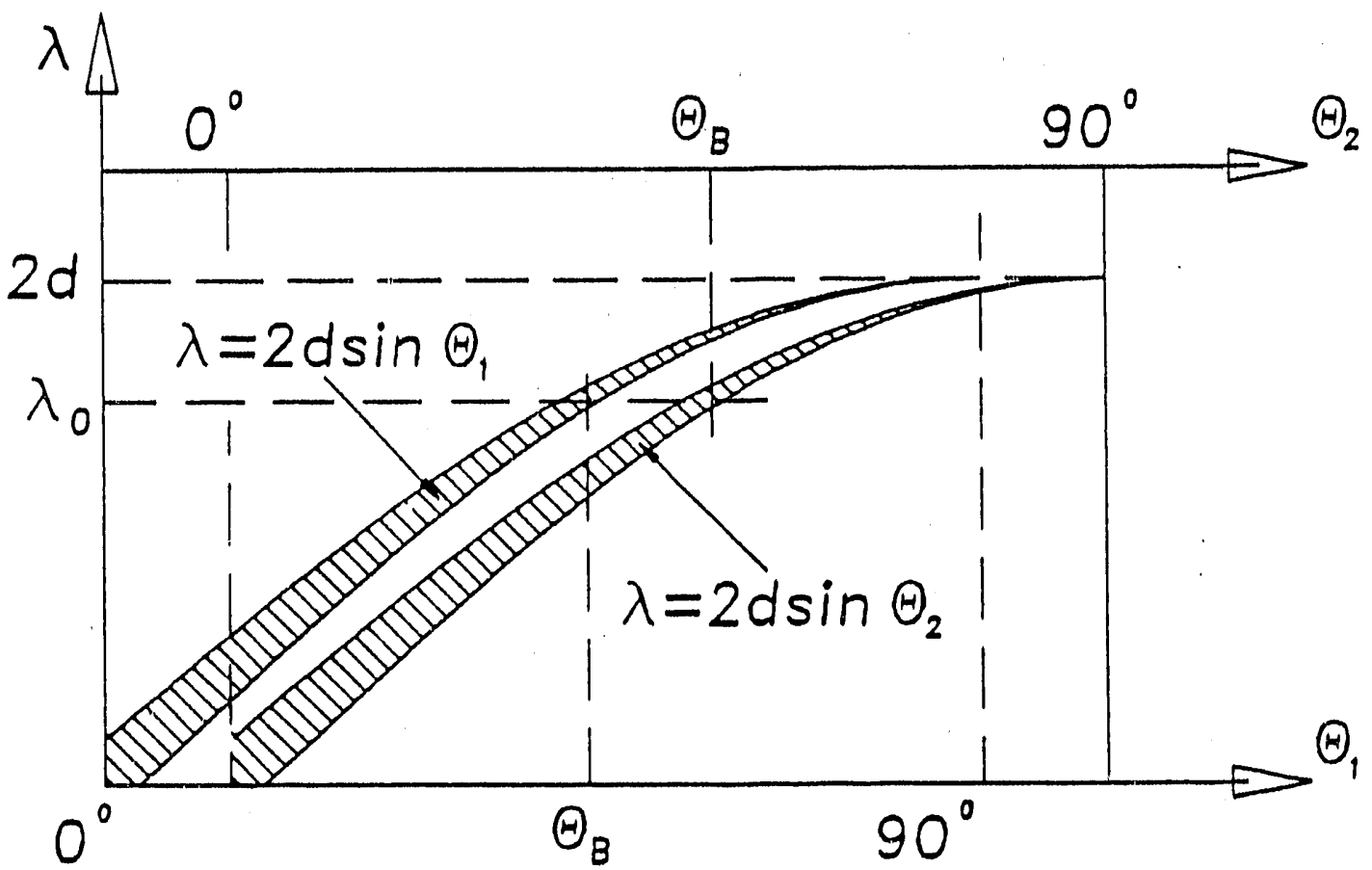

Fig. 15 Dumond representation for parallel double-crystal spectrometer.

We see that if a detector is used such that it will accept an angular spread smaller than that of the incident $x$-ray beam but larger than $w$, the wavelength resolution is limited by the detector for the single-crystal and parallel double-crystal cases, and by $w$ for the anti-parallel double-crystal case. The difference between the former two arrangements lies in the fact that by slightly offsetting in $\theta$ the two crystals in the parallel double-crystal system, a narrower band of intensity in the $\theta-\lambda$ plane than the single-crystal DuMond band is passed (see Fig. 16). Hence, a more definite relation between $\lambda$ and $\theta$ exists for the emerging $x$-rays. These may then be used to probe a third crystal, arranged parallel to the first two (which was what DuMond originally had in mind when he approached $x$-ray optics through plots in the $\theta-\lambda$ plane [7]). An asymmetric single-crystal diffraction will provide somewhat the same effect. 


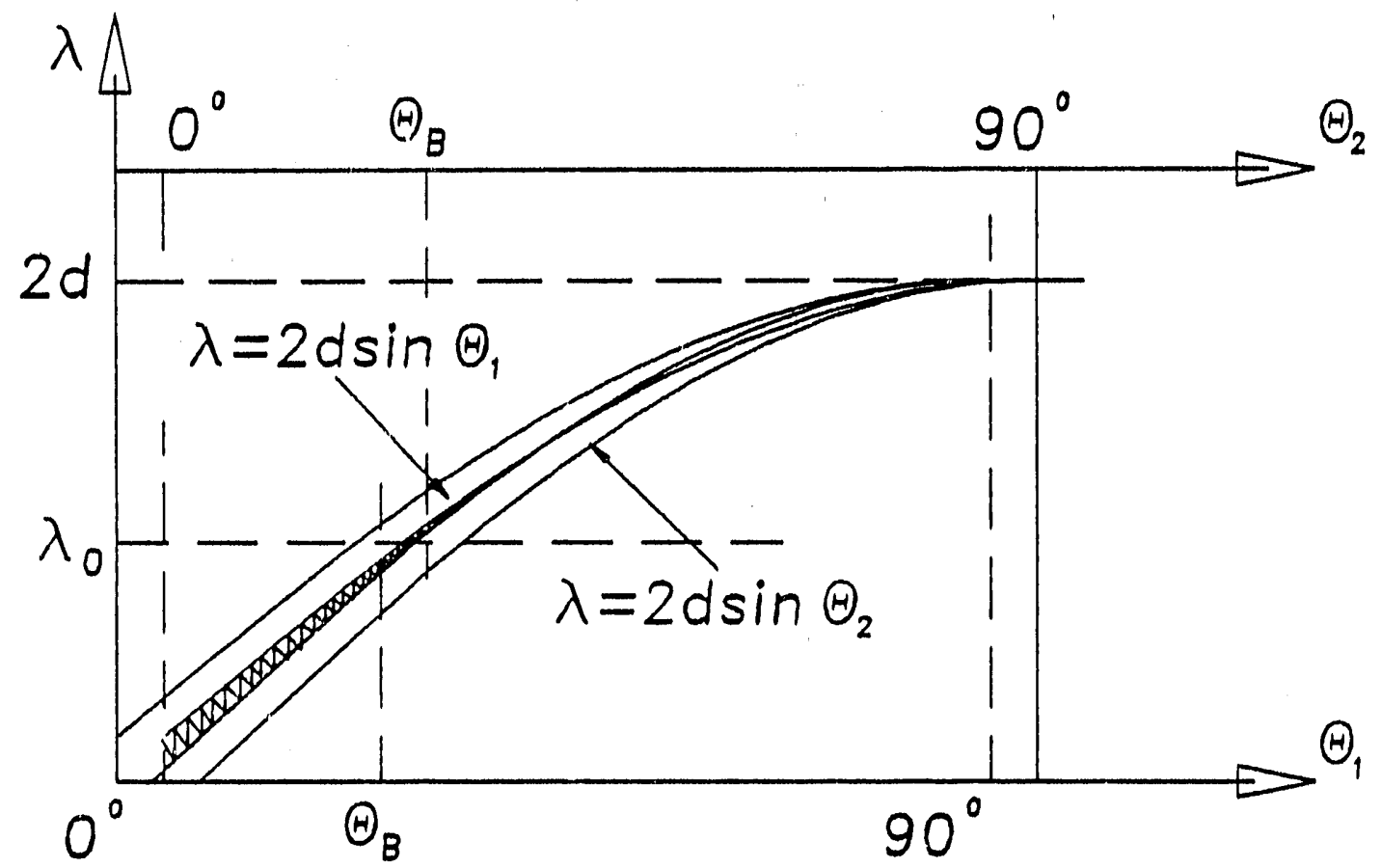

Fig. 16 DuMond representation for parallel double-crystal spectroneter with the crystals slightly offset in $\theta$

The parallel double-crystal spectrometer is the most popular instrument used for wavelength selection at synchrotron $x$-ray beam lines. It is preferred over the single-crystal spectrometer, since the emerging beam is parallel to the incident beam. While offering poorer wavelength resolution compared with the anti-parallel arrangement, the parallel spectrometer transmits more intensity and is much simpler to design.

If the two crystals in the parallel double-crystal arrangement are of different types (e.g., possess different d-spacings), then some dispersion will indeed result when the crystals are oriented at their respective Bragg angles $\theta_{B 1}$ and $\theta_{B 2}$ for $\lambda_{0}$ :

$$
\frac{d \theta}{d \lambda}=\frac{\tan \theta_{B 1}-\tan \theta_{B 2}}{\lambda_{0}} \text {. }
$$

Fig. 17 shows the component single-crystal DuMond bands for this system. An improvement in wavelength resolution is realized over the typical parallel arrangement (crystals of same type), while giving worse resolution than the antiparallel arrangement. 


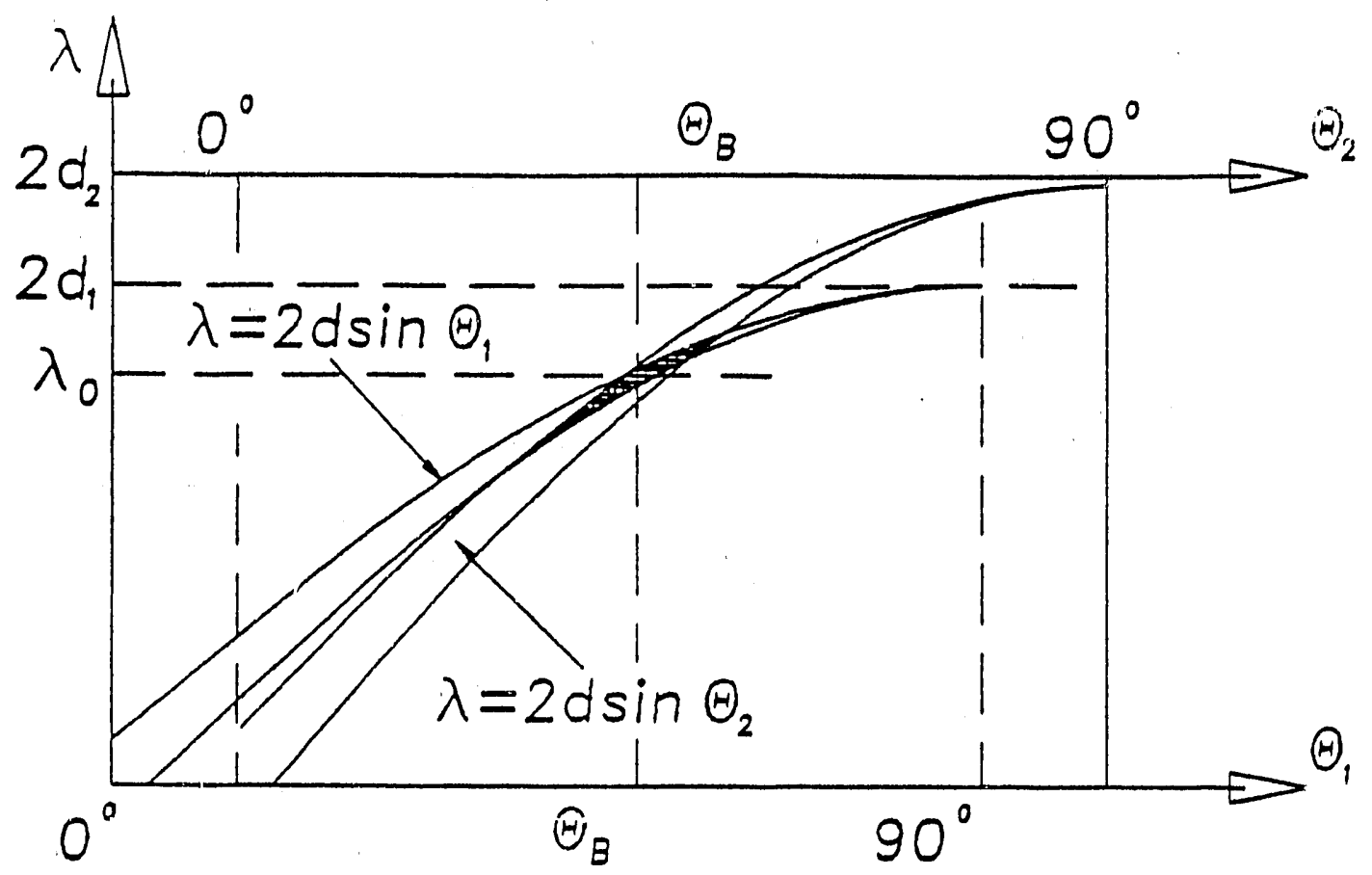

Fig. 17 DuMond representation for parallel double-cyrstal spectrometer for two crystals with different d-spacings.

For angles very close to $\theta_{\mathrm{B}}$, Bragg's law shows an approximately linear relationship between $\theta$ and $\lambda$ (see Eq.11). Hence, a plot of $d \lambda$ vs. $d \theta$ gives a line of constant slope $\lambda_{0} \cot \theta_{B}$. For a small spread $d \lambda$ about $\lambda_{o}$, the Darwin width $w$ hardly varies for reflection from a set of crystal lattice planes. Hence, a plot of total reflection in the $d \theta-d \lambda$ plane yields a linear band (see Fig. 18). This band constitutes the windows of acceptance and emergence for this crystal oriented at $\theta_{B}$ to incident $x$-rays centered in wavelength at $\lambda_{0}=2 d \sin \theta_{B}$.

If a crystal is oriented at $\theta_{B}$ with respect to the horizontal plane of a synchrotron source, we can call $d \theta$ the vertical angular divergence $y^{\prime}$. The band shown in Fig. 17 can be extended over all vertical displacements y, yielding a slab of acceptance for this crystal in M-K space (see Fig. 19). The M-K transformation matrices for the crystal are $\left[\begin{array}{ccc}-1 & 0 & 0 \\ 0 & -1 & 0 \\ 0 & 0 & 1\end{array}\right]$ for $\left(y, y^{\prime}, \lambda\right)$ falling within the confines of 
the slab, and $\left[\begin{array}{lll}0 & 0 & 0 \\ 0 & 0 & 0 \\ 0 & 0 & 0\end{array}\right]$ otherwise (an M-K matrix transforms $\left[\begin{array}{c}y_{i n} \\ y_{1 n}^{\prime} \\ \lambda_{i n}\end{array}\right]$ to $\left[\begin{array}{c}y_{\text {emerging }} \\ y_{1}^{\prime} \text { emerging } \\ \lambda_{\text {emerging }}\end{array}\right]$.

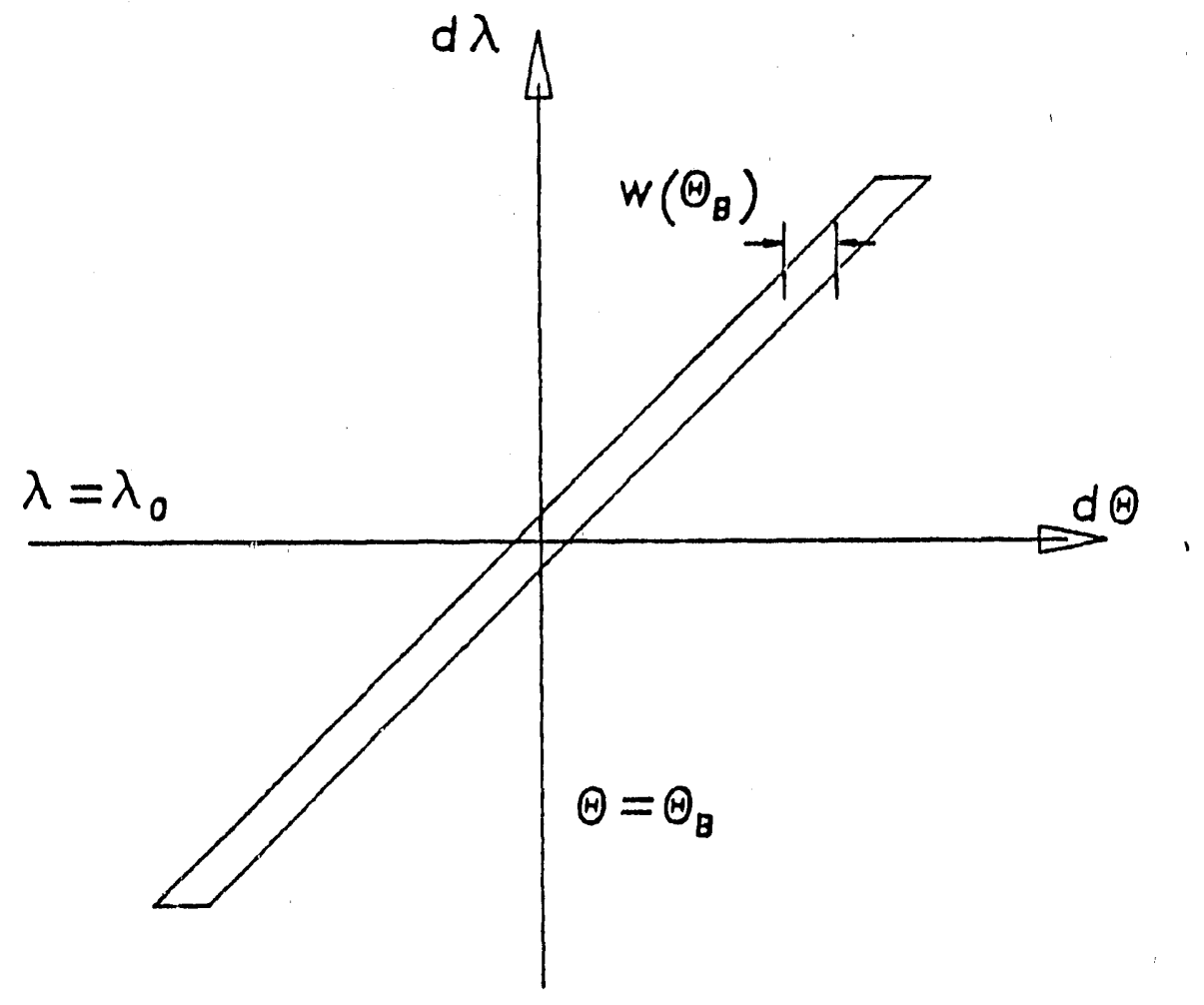

Fig. 18 Window of acceptance (emergence) for a single crystal in the $d \theta-d \lambda$ plane.

The crystal effectively acts as a mirror for photons whose M-K coordinates are those of a point with:n the slab; the optical axis is changed in direction by $2 \theta_{B}$ from the horizontal plane.

The photon phase ellipse may also be extended along the wavelength-, or $\lambda-$, axis for small excursions $d \lambda$ (for large excursic is, the phase ellipse will change, as will the photon number $N$ ). Hence, the source can be represented as an elliptical cylinder in M-K space (see Fig. 20). 


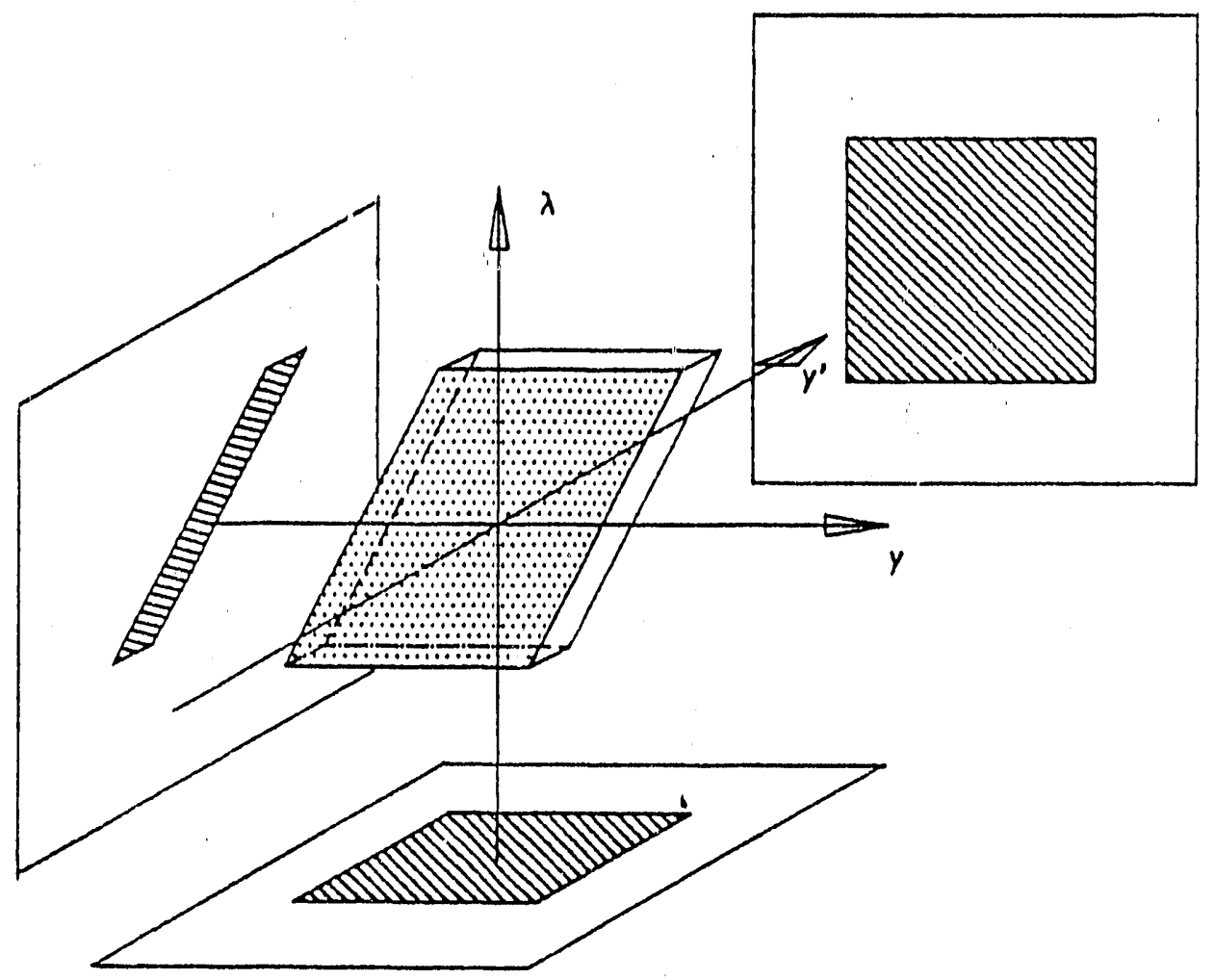

Fig. 19 M-K space of acceptance for a single crystal.

If a crystal is placed in the path of a storage ring $x$-ray beam oriented at $\theta_{B}$ to the horizontal plane for $\lambda_{0}$, its $\mathrm{M}-\mathrm{K}$ slab will intersect with the elliptical cylinder representing the beam at that particular point $z$ in an elliptical slab of emergence (see Fig. 21). This slab will have the angular spread $\Delta y_{m}$ ' about $\theta_{B}$ of the photon phase ellipse at $z$, and a wavelength spread about $\lambda_{0}$ of $\Delta \lambda_{m}=\lambda_{0} \cot \theta_{B}\left(\Delta y_{m}{ }^{2}+w^{2}\right)^{1 / 2}$. For $\Delta y_{m}{ }^{\prime}>>w, \Delta \lambda_{m} \simeq \lambda_{0} \Delta y_{m}{ }^{\prime} \cot \Theta_{B}$. The beam intensity passed is proportional to the elliptical slab volume, which is approximately $S_{s y} \lambda_{o} w \cot \Theta_{B}$. The beam height is $\Delta y_{m}$, the width of the photon phase ellipse along with $y$-axis.

If a slit of vertical width $\Delta y_{s}=2 y_{s}<2 y_{m}$ is placed at $z$ next to the crystal, the area of the phase ellipse passed is

$S_{u}=\left(2 S_{s y} / \pi W^{2}\right)\left(y_{s}\left[W^{2}-y_{s}{ }^{2}\right]^{1 / 2}+W^{2} \sin ^{-1}\left[y_{s} / W\right]\right)$, where $W=y_{m}$, and the angular width of this area is $\Delta \lambda_{s}{ }^{\prime}=2 y_{s}^{\prime}=\left(2 y_{s}\left[y_{m}{ }^{\prime 2} z-\alpha \epsilon\right]+\left[2 S_{s y} / \pi\right]\left[W^{2}-y_{s}{ }^{2}\right]^{1 / 2}\right) / W^{2}$. The wavelength spread passed is $\Delta \lambda_{s}=\lambda_{0} \cot \theta_{B}\left(\Delta y_{s}{ }_{s}{ }^{2}+w^{2}\right)^{1 / 2}$ about $\lambda_{0}$. For $\Delta y_{s}{ }^{\prime}$ $>>w, \Delta \lambda_{s}=\lambda_{o} \Delta y_{s}{ }^{\prime} \cot \theta_{B}$. The beam intensity passed is proportional to the elliptical slab chunk volume (see Fig. 21), which is approximately $S_{U} \lambda_{0} w \cot \Theta_{B}$. The beam height is narrowed down to the slit width $\Delta \mathrm{y}_{\mathrm{s}}$. 


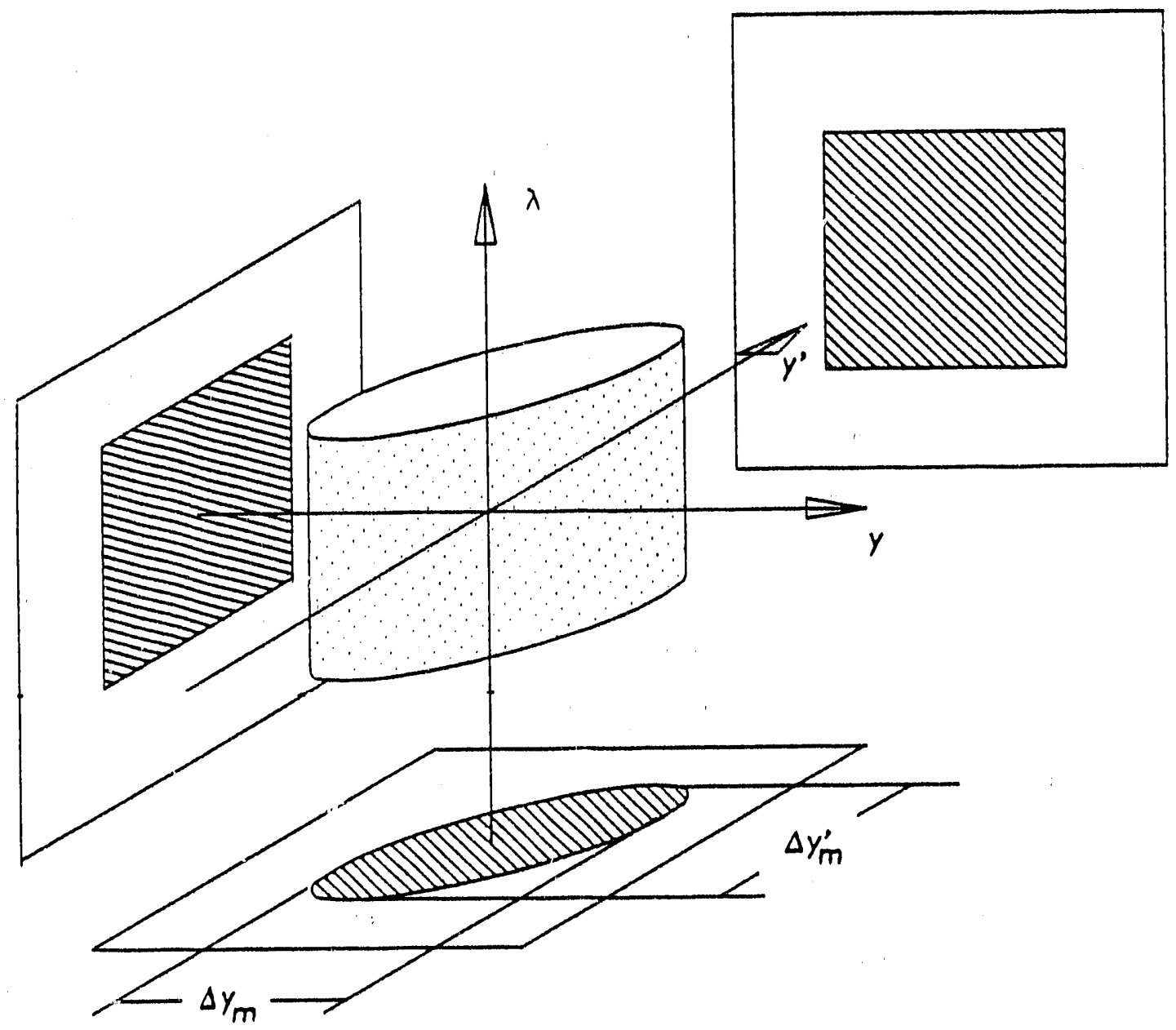

Fig. $20 \mathrm{M}-\mathrm{K}$ space representation of source.

\subsubsection{Comparision of Optical Arrangements}

In the following figures, the performance of four optical systems with regard to energy resolution and photon number passed are evaluated through analyses of orientations of M-K space volumes: (I) source plus one crystal located at $\mathrm{z}$, (II) source plus one slit and one crystal located at $z$, (III) source plus two anti-parallel crystals located at $z$, and (IV) source plus two parallel crystals with offset $d$-spacin ¿s located at $z$. Table I summarizes the results. 


\section{System I: Source Plus One Crystal Located at $z$}

elliptical cylinder volume available $=S_{s y} \Delta \lambda_{m}=S_{s y} \lambda_{0} \cot \theta_{B}\left(\Delta y_{m}^{\prime}{ }^{2}+w^{2}\right)^{1 / 2}$ synchrotron bandwidth $=\Delta \lambda_{\mathrm{m}}$

elliptical slab volume accepted $=S_{s y} d \lambda=S_{s y} \lambda_{0} w \cot \theta_{B}$

$$
\begin{aligned}
& 8 \text { photons transmitted }=\frac{s_{s y} \lambda_{0} w C O t \theta_{B}}{S_{a y} \lambda_{0} \cot \theta_{B}\left(\Delta y_{m}^{\prime 2}+w^{2}\right)^{1 / 2}}=\frac{w}{\left(\Delta y_{m}^{\prime 2}+w^{2}\right)^{1 / 2}} \\
& =\frac{w}{\Delta y_{m}^{\prime}}, w<<\Delta y_{m}^{\prime} \\
& \text { wavelength resolution }=\Delta \lambda_{m}=\lambda_{0} \cot \theta_{B}\left(\Delta y_{m}^{\prime 2}+w^{2}\right)^{1 / 2} \\
& \approx \lambda_{0} \Delta y_{m}^{\prime} \cot \theta_{B}, w<<y_{m}^{\prime} \\
& \text { energy resolution }=E_{0} \cot \theta_{B}\left(\Delta y_{m}^{\prime 2}+w^{2}\right)^{1 / 2} \approx E_{0} \Delta y_{m}^{\prime} \cot \theta_{B}, w<<\Delta y_{m}^{\prime} \\
& \text { photon \# passed }=N\left(\lambda_{0}\right) \times \text { (synchrotron bandwidth) } x_{1}(z \text { trans. }) \\
& =N\left(\lambda_{0}\right) \lambda_{0} \cot \theta_{B}\left(\Delta y_{m}^{\prime 2}+w^{2}\right)^{1 / 2} \frac{w}{\left(\Delta y_{m}^{\prime 2}+w^{2}\right)^{1 / 2}} \\
& \text { beam height passed }=\Delta y_{m}
\end{aligned}
$$

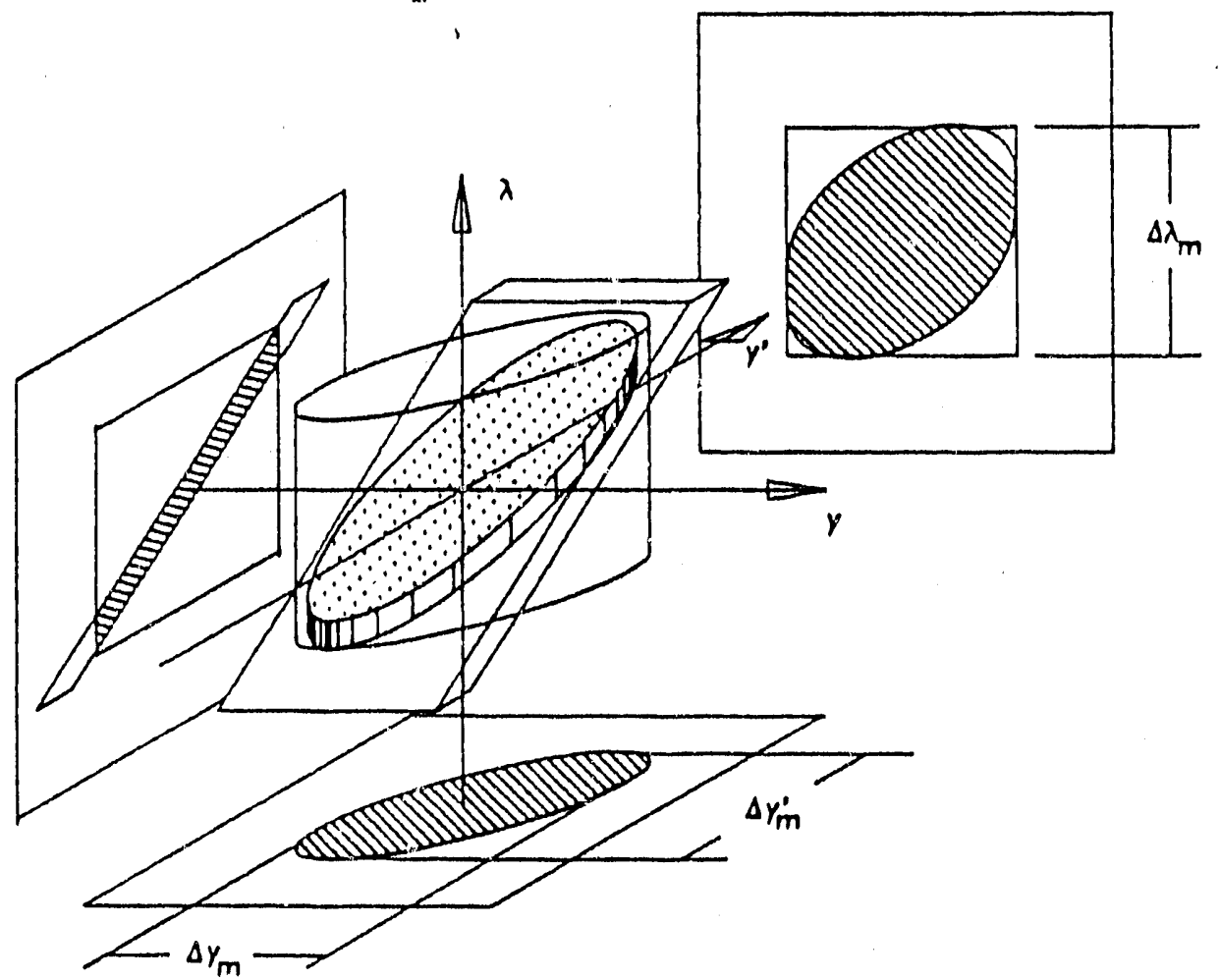

Fig. 21 M-K space representation of source plus one crystal (system I). 


\section{System II: Source Plus One Slit and One Crystal Located at Z}

elliptical cylinder volume available $=S_{s y} \Delta \lambda_{m}=S_{s y} \lambda_{0} \cot \Theta_{B}\left(\Delta y_{m}^{\prime}{ }^{2}+w^{2}\right)^{1 / 2}$ synchrotron bandwidth $=\Delta \lambda_{\mathrm{m}}$

elliptical slab volume accepted $=S_{u} d \lambda=S_{u} \lambda_{0} w \cot \theta_{B}$

\& photons transmitted $=\frac{S_{u} W}{S_{z y}\left(\Delta y_{m}^{\prime}+w^{2}\right)^{1 / 2}}$

$$
\approx \frac{S_{u} w}{S_{s y} \Delta y_{m}^{\prime}}, w<\Delta y_{m}^{\prime}
$$

energy resolution $=E_{0} \cot \theta_{B}\left(\Delta y_{s}^{\prime 2}+w^{2}\right)^{1 / 2} \approx E_{0} \Delta y_{s}^{\prime} \cot \theta_{B}, w<<\Delta y_{s}^{\prime}$

photon \# passed $=N\left(\lambda_{0}\right) \times$ (synchrotron bandwidth) $\times(z$ trans.)

beam height passed $=\Delta y_{s}$

$$
\begin{aligned}
& =N\left(\lambda_{0}\right) \lambda_{0} \cot \theta_{B}\left(\Delta y_{m}^{\prime 2}+w^{2}\right)^{1 / 2} \frac{S_{u} w}{S_{s y}\left(\Delta y_{m}^{\prime 2}+w^{2}\right)^{1 / 2}} \\
& =N\left(\lambda_{0}\right)\left(\frac{S_{y}}{S_{s y}}\right) \lambda_{0} w \cot \theta_{B}=N\left(E_{0}\right)\left(\frac{S_{u}}{S_{s y}}\right) E_{0} w \cot \theta_{B}
\end{aligned}
$$

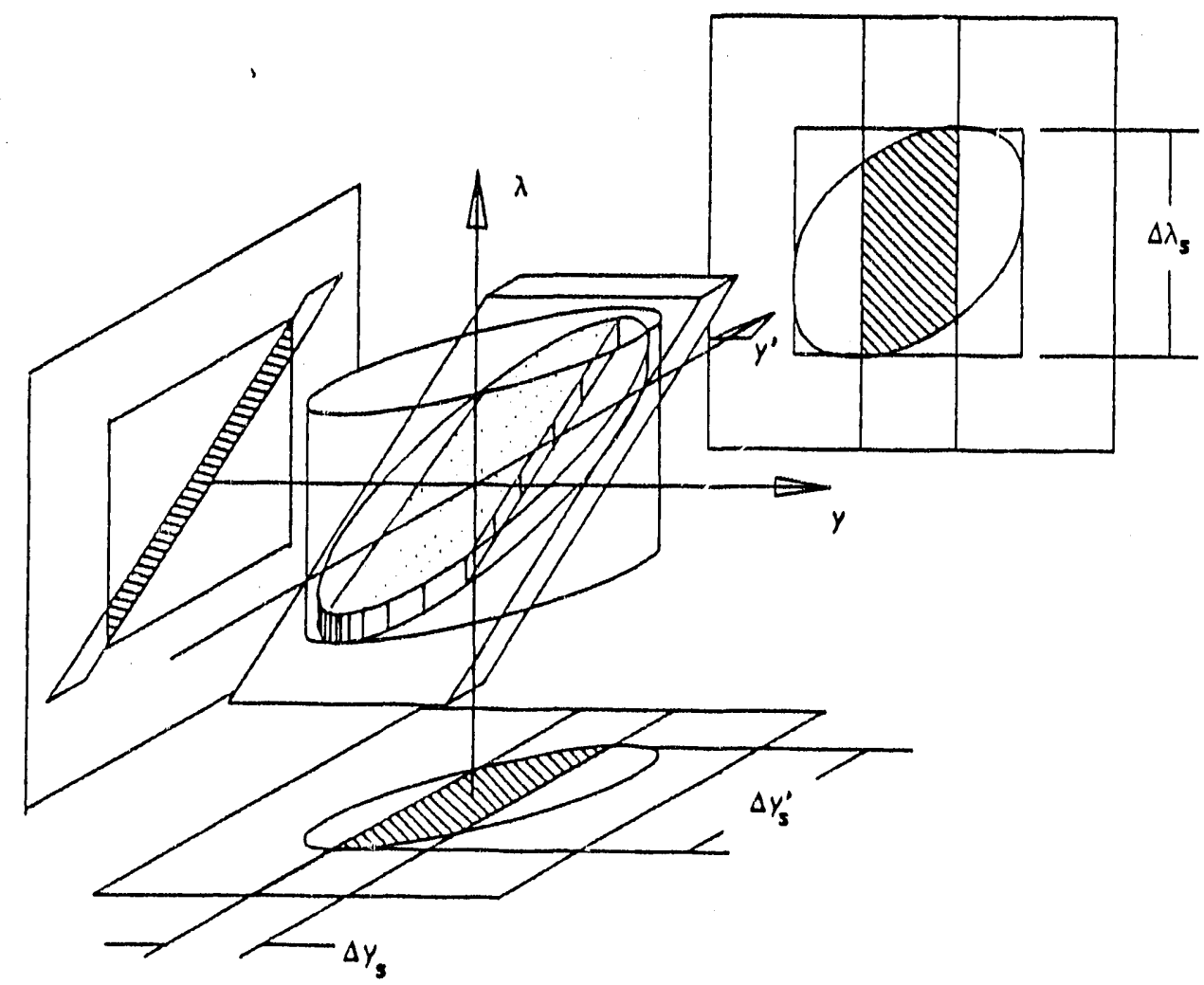

Fig.22 M-K space representation of source plus one slit and one crystal , 


\section{System III: Source Plus Two Anti-Parallel Crystais Located at z}

elliptical cylinder volume available $=S_{s y} \Delta \lambda_{m}=S_{s y} \lambda_{o} \cot \Theta_{B}\left(\Delta y_{m}^{\prime}{ }^{2}+w^{2}\right)^{1 / 2}$ synchrotron bandwidth $=\Delta \lambda_{\mathrm{m}}$

$$
\begin{aligned}
\text { pencil volume accepted } & =\text { (area of rhombus endpiece }) \times \text { (pencil length) } \\
& =\left(\frac{1}{2} w d \lambda\right) \Delta y_{0}=\left(\frac{1}{2} w \lambda_{0} w C o t \theta_{b}\right)\left(\frac{4 S_{s y}}{\pi \Delta y_{m}^{\prime}}\right) \\
\text { \& photons transmitted } & =\frac{2 w^{2}}{\pi \Delta y_{m}^{\prime}\left(\Delta y_{m}^{\prime 2}+w^{2}\right)^{1 / 2}} \\
& \approx \frac{2 w^{2}}{\pi \Delta y_{m}^{\prime 2}}, w<\left\langle\Delta y_{m}^{\prime}\right. \\
\text { energy resolution } & =E_{0} w \cot \theta_{B} \\
\text { photon \# passed } & =N\left(\lambda_{0}\right) \times(s y n c h r o t r o n \text { bandwidth }) x(z \text { trans. }) \\
& =N\left(\lambda_{0}\right) \lambda_{0} \cot \theta_{B}\left(\Delta y_{m}^{\prime 2}+w^{2}\right)^{1 / 2} \frac{2 w^{2}}{\pi \Delta y_{m}^{\prime}\left(\Delta y_{m}^{\prime 2}+w^{2}\right)^{1 / 2}} \\
& =N\left(\lambda_{0}\right)\left(\frac{2 w}{\pi \Delta y_{m}^{\prime}}\right) \lambda_{0} w \operatorname{wot} \theta_{B}=N\left(E_{0}\right)\left(\frac{2 w}{\pi \Delta y_{m}^{\prime}}\right) E_{0} w \cot \theta_{b} \\
\text { beam height passed } & =\Delta y_{0}
\end{aligned}
$$

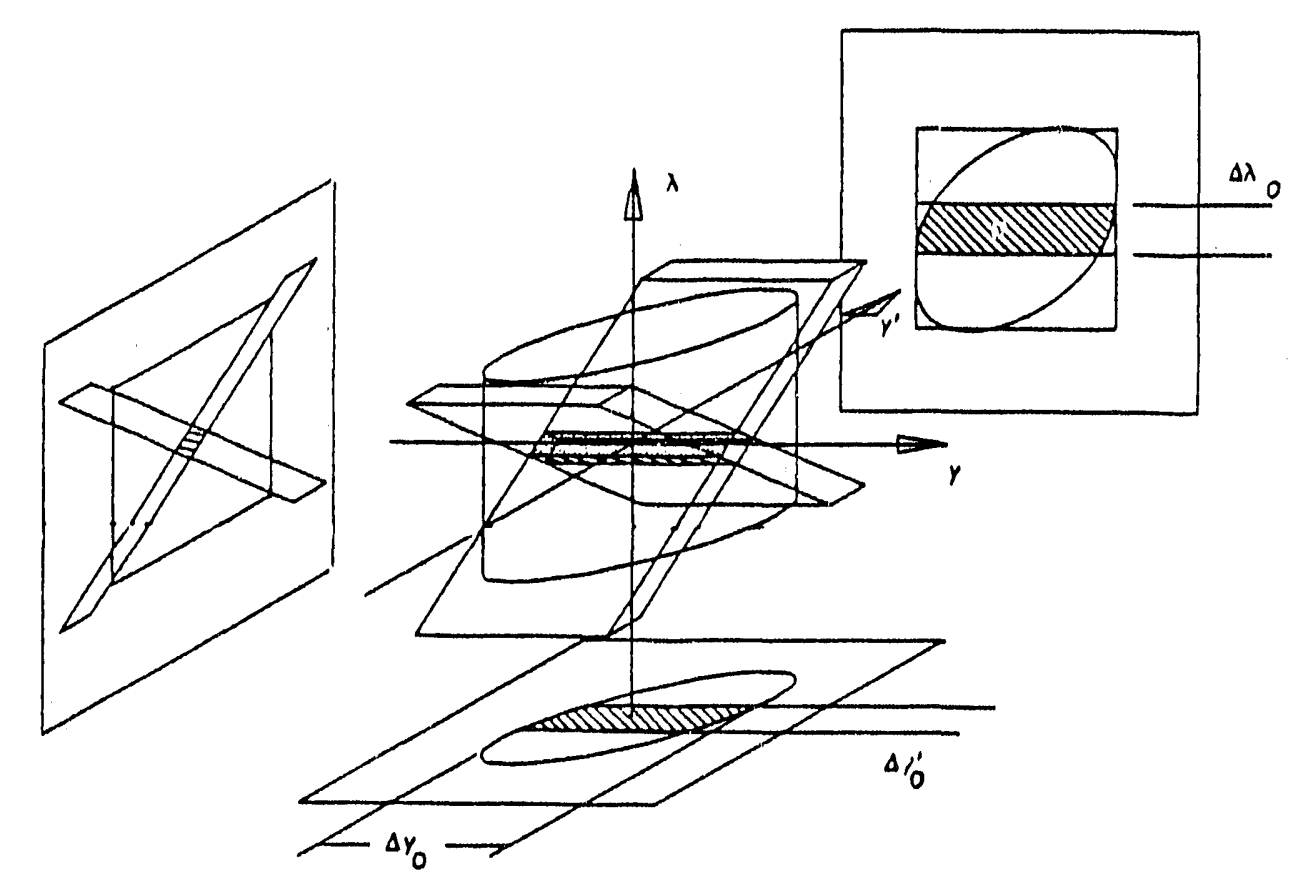

Fig. 23 M-K space representation of source plus two identical anti-parallel crystals. 


\section{System IV: Source Plus Two Offset-d Parallel Crystals Located at $z$}

(crystal 1 is first, crystal 2 is second)

elliptical cylinder volume available $=S_{s y} \Delta \lambda_{m}=S_{s y} \lambda_{0} \cot \Theta_{B 1}\left(\Delta y_{m}^{\prime}{ }^{2}+w_{1}^{2}\right)^{1 / 2}$ synchrotron bandwidth $=\Delta \lambda_{\mathrm{m}}$

elliptical slab chunk volume accepted $=\frac{\lambda_{0} w_{1} w_{2} \Delta y_{0}}{\tan \theta_{B 1}-\tan \theta_{B 2}}$

$$
=\frac{4 s_{s y} \lambda_{0} w_{1} w_{2}}{\pi \Delta y_{m}^{\prime}\left(\tan \theta_{B 1}-\tan \theta_{B 2}\right)}
$$

\& photons transmitted $=$

$$
=\frac{4 w_{1} w_{2}}{\pi \Delta y_{m}^{\prime} \cot \theta_{B 1}\left(\Delta y_{m}^{\prime 2}+w_{1}^{2}\right)^{1 / 2}\left(\tan \theta_{B 1}-\tan \theta_{B 2}\right)}
$$

energy resolution $=\frac{E_{0}\left(w_{1}+w_{2}\right)}{\tan \theta_{B 1}-\tan \theta_{B 2}}=\frac{E_{0}\left(w_{1}+w_{2}\right) \cot \theta_{B 1}}{\left(1-\frac{\tan \theta_{B 2}}{\tan \theta_{B 1}}\right)}$

photon \# passed $=N\left(\lambda_{0}\right) \times$ (synchrotron bandwidth) $\times$ (8 trans.)

$=N\left(\lambda_{0}\right) \lambda_{0} \cot \theta_{B 1}\left(\Delta y_{m}^{\prime 2}+w_{1}^{2}\right)^{1 / 2}$

$\frac{4 w_{1} w_{2}}{\pi \Delta y_{m}^{\prime} \cot \theta_{B 1}\left(\Delta y_{m}^{\prime 2}+w_{2}^{2}\right)^{1 / 2}\left(\tan \theta_{B 1}-\tan \theta_{B 2}\right)}$

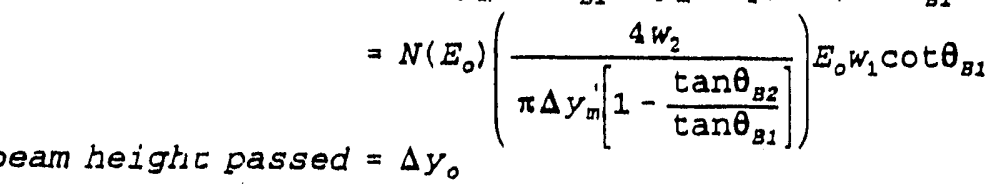

$\begin{aligned} & =N\left(E_{0}\right)\left(\frac{4 W_{2}}{\pi \Delta y_{m}^{\prime}\left[1-\frac{\tan \theta_{B 2}}{\tan \theta_{B 1}}\right]}\right) E_{0} w_{1} \cot \theta_{B 1} \\ \text { beam height passed } & =\Delta y_{0}\end{aligned}$ 


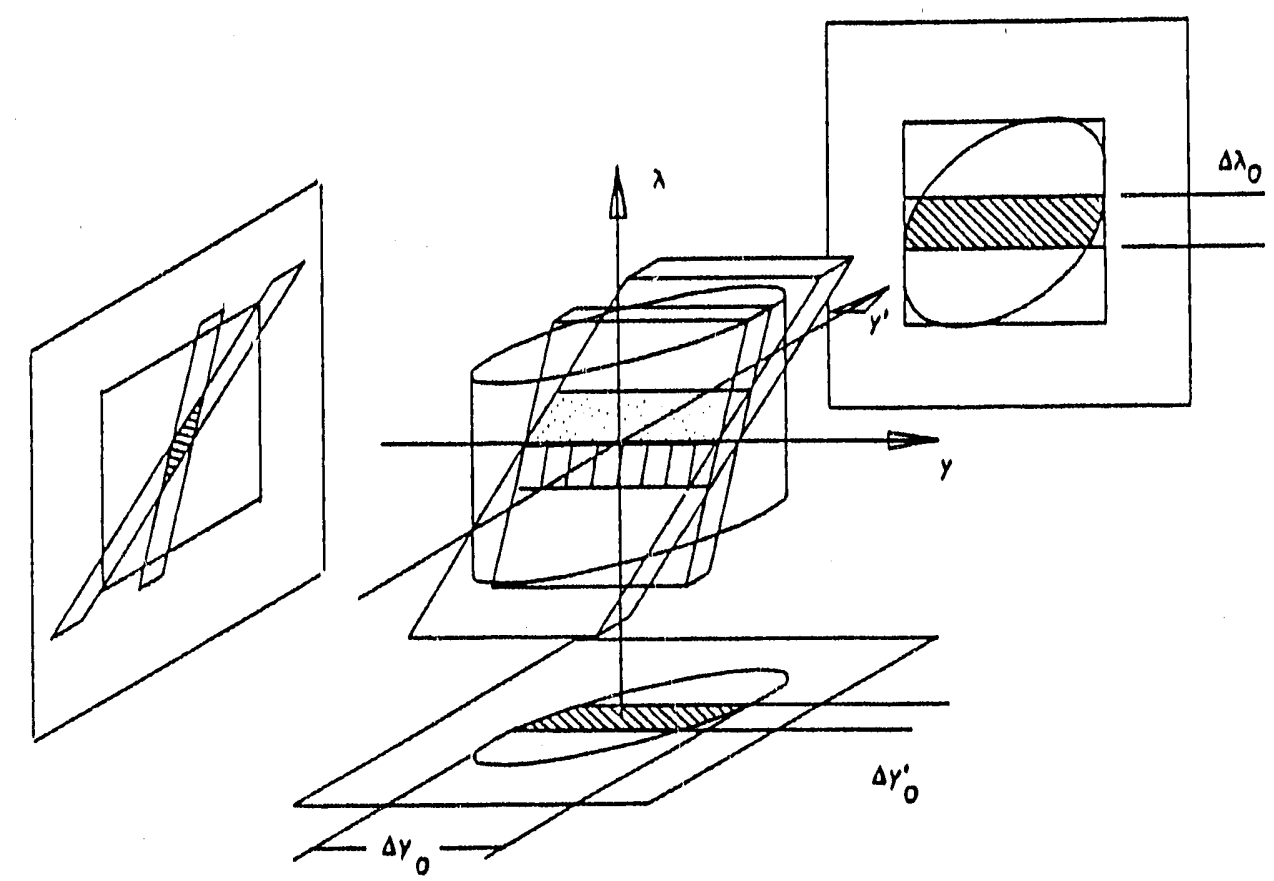

Fig. $24 \mathrm{M}-\mathrm{K}$ space representation of source plus two parallel crystals with different $d$-spacings (system IV)

Table I: Summary of Characteristics of Optical Systems

(we assume that $w, w_{2}<<\Delta y_{m}^{\prime}, \Delta y_{g}^{\prime}$; for System II, $w$ is the Darwin width of the first crystal [Bragg angle $=\Theta_{B}$; and $w_{2}$ is the Darwin width of the second crystal (Bragg angle $=\theta_{B 2}$ )

\begin{tabular}{|c|c|c|c|}
\hline System & Photon \# Passed & Energy Resolution & Beam Height Passed \\
\hline I & $N\left(E_{0}\right) E_{0} \omega \cot \theta_{B}$ & $E_{a} y_{m}^{\prime} \cot \theta_{B}$ & $\Delta y_{m}$ \\
\hline II & $N\left(E_{0}\right)\left(\frac{S_{u}}{S_{s y}}\right) E_{o} w C O t \theta_{B}$ & $E_{0} y_{s}^{\prime} \cot \theta_{B}$ & $\Delta y_{s}$ \\
\hline IV & $N\left(E_{0}\right)\left(\frac{4 W_{2}}{\pi \Delta y_{n}^{\prime}\left[1-\frac{\tan \theta_{B z}}{\tan \theta_{n}}\right)}\right) E_{0}$ weott & $\frac{E_{0}\left(w+w_{2}\right) \cot \theta_{B}}{\left(1-\frac{\tan \theta_{B z}}{\tan \theta_{B}}\right)}$ & $\Delta y_{0}$ \\
\hline III & $\left(E_{0}\right)\left(\frac{2 w}{\pi \Delta y_{m}^{\prime}}\right) E_{o} w \cot \theta_{a}$ & $E_{0} w C o t \theta_{B}$ & $\Delta y_{0}$ \\
\hline
\end{tabular}




\section{$\underline{\text { Ratios of Parameters of I:II:IV:III }}$}

$$
\begin{aligned}
& \text { photon \# passed : } \quad 1: \frac{S_{u}}{S_{s y}}: \frac{4 w_{2}}{\pi \Delta y_{m}^{\prime}\left(1-\frac{\tan \theta_{B 2}}{\tan \theta_{B}}\right)}: \frac{2 w}{\pi \Delta y_{m}^{\prime}} \\
& \text { energy resolution: } \quad \Delta y_{m}^{\prime}: \Delta y_{s}^{\prime}: \frac{w+w_{2}}{\left(1-\frac{\tan \theta_{B 2}}{\tan \theta_{B}}\right)} ; w
\end{aligned}
$$

\subsection{Focussing Perfect Crystals}

Many user applications require high photon intensities. One method of achieving this is to focus the $x$-ray beam. The incident beam can be focussed using a curved perfect crystal. Consider a crystal curved to a radius $R / 2$ focussing a vertically-diverging beam, where $R$ is the radius of curvature of the diffracting lattice planes (Johannson-type curved crystal; see Fig. 25). The source-to-crystal distance $p_{o}$ and the crystal-to-focus distance $q_{o}$ are equal only if the diffraction is symmetric. The source and focal points lie on the Rowland circle of radius $R / 2$, of which the crystal surface forms an arc component [5].

Assuming the source $S$ is a point source, one can see from geometrical considerations that the entire beam is focussed at the point $F$. The $M-K$ transformation matrix for this crystal is $\left(\begin{array}{ccc}-1 & 0 & 0 \\ 1 / f & -1 & 0 \\ 0 & 0 & 1\end{array}\right)$ for a symmetric diffraction, where the focal length is determined from

$$
\frac{1}{f}=\frac{1}{p_{0}}+\frac{1}{q_{0}}
$$

Note that the optical axis has changed in direction by $2 \theta_{B}$, where $\theta_{B}$ is the angle of incidence of the optical axis with the diffracting lattice planes of the crystal and is determined from $\lambda_{0}=2 d \sin \theta_{B}$, with $\lambda_{0}$ the central incident wavelength and $d$ the spacing of these planes. 


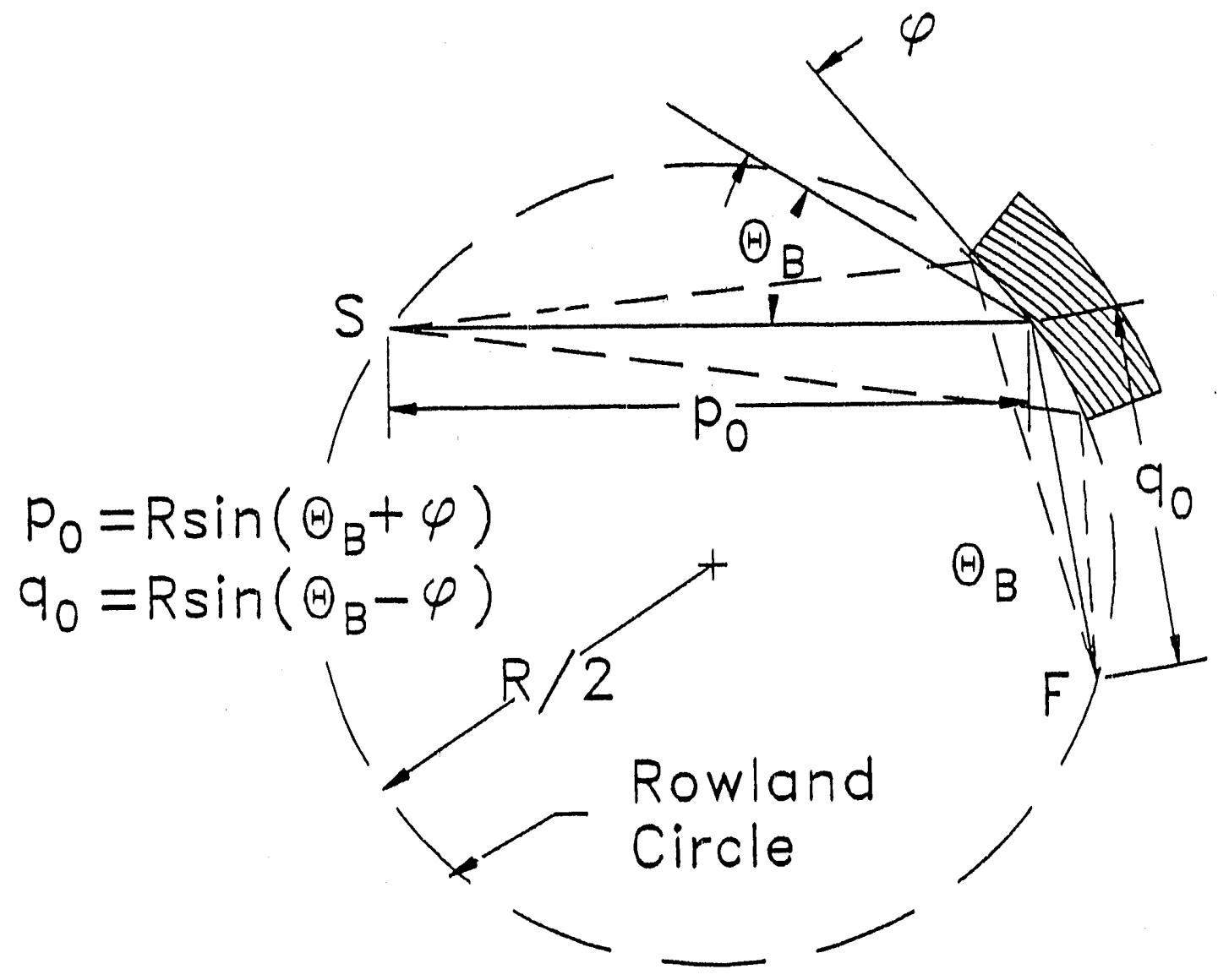

Fig. 25 Single curved crystal focusing geometry.

Fig. 26 shows the transformation of a synchrotron source beam in M-K space for a symmetric diffraction. The projection of the slab volume $V_{f}$ representing the focussed beam onto the $y-y^{\prime}$ plane is a right ellipse. The height of the focussed beam is the vertical "source size" $\Delta y_{0}$. Notice that the slab of acceptance is that for a flat perfect crystal, skewed in the $y$-y' plane. Also notice the narrower wavelength spread than that obtained from a flat crystal diffraction. This is because the angles of incidence of the ray components of the beam with this crystal do not stray far from $\theta_{B}$, as would have been the case for incidence with a flat crystal. 


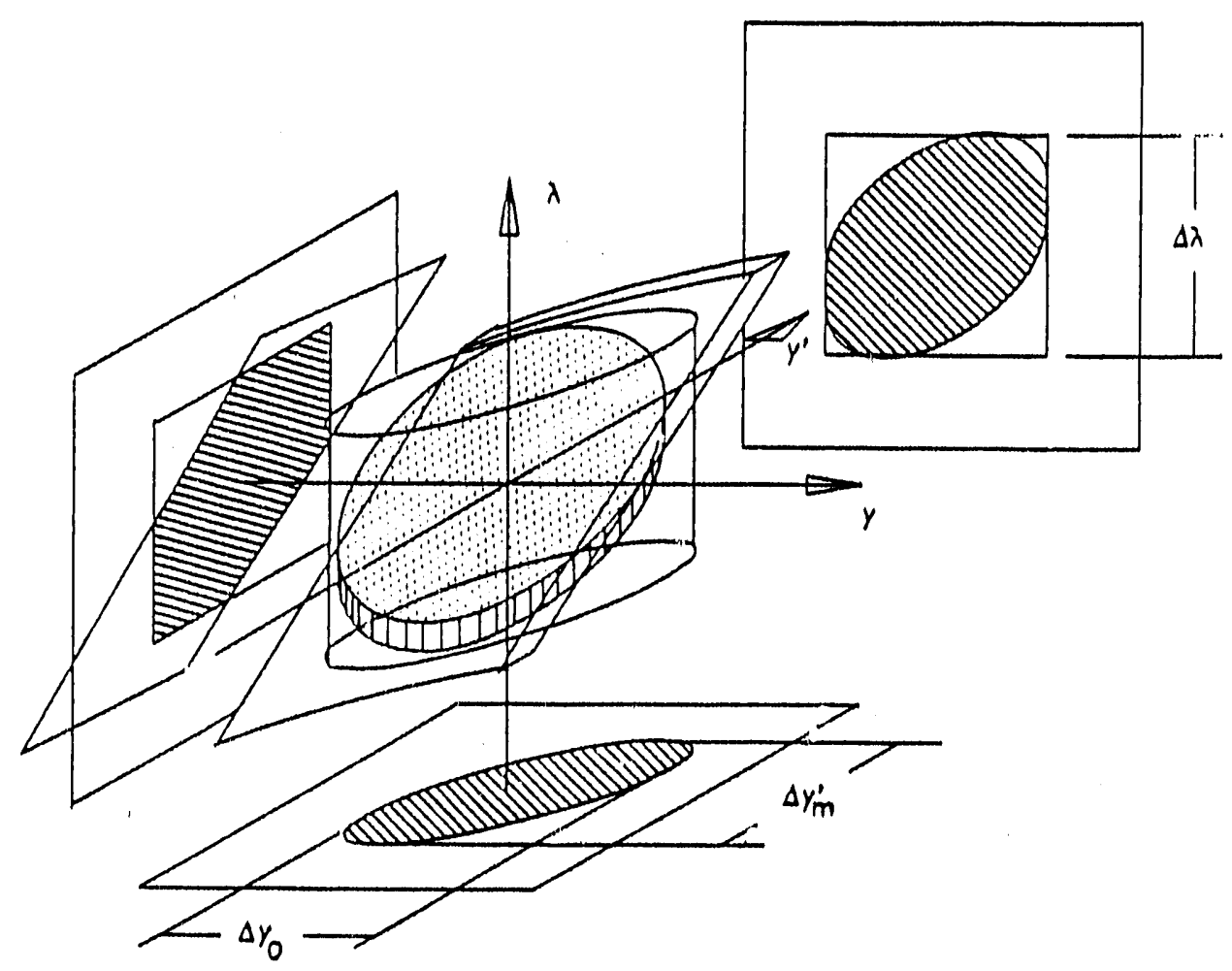

Fig. 26 M-K space representation of curved crystal focusing.

\subsection{Geometric Optics}

The transmission of a grating is much larger than that of a crystal. The diffraction condition, unlike the Bragg condition, can be satisfied for any incident ray with wavelength $0<\lambda<\mathrm{d}$ by some diffracted ray in some diffracted order (either positive or negative). Here $d$ is the period of the grating groove spacing, which is assumed to be uniform. This is illustrated in Fig. 27, in which the allowed range of diffracted wavelengths for first and second diffracted orders is plotted as a function of the grazing angle of the incident ray. These wavelength ranges correspond to all physically allowed diffracted rays, which are those that satisfy the grating equation

$$
\sin \alpha+\sin \beta=n \lambda / d \text {. }
$$

for $-90^{\circ}<\beta<90$ and $n= \pm 1, \pm 2 . \alpha$ and $\beta$ are the angle of incidence of the incoming ray and the angle of exit of the outgoing diffracted ray, respectively, with respect to the grating normal, $\mathrm{n}$ is the order of diffraction, and $\lambda$ is the photon 
wavelength. Of course, Eq.(16) applies to all diffracted orders $n$, where 4 integer; the value of $\lambda / d$ for $\theta=90^{\circ}$ in Fig. 27 moves progressively to lower valuls as the diffracted order $n$ increases. Fig. 27 is a DuMond plot for a grating: it displays the transmission of a diffraction grating. All rays outside the hatched areas correspond to diffraction conditions in which the diffracted ray is directed into the bulk of the grating and is absorbed.

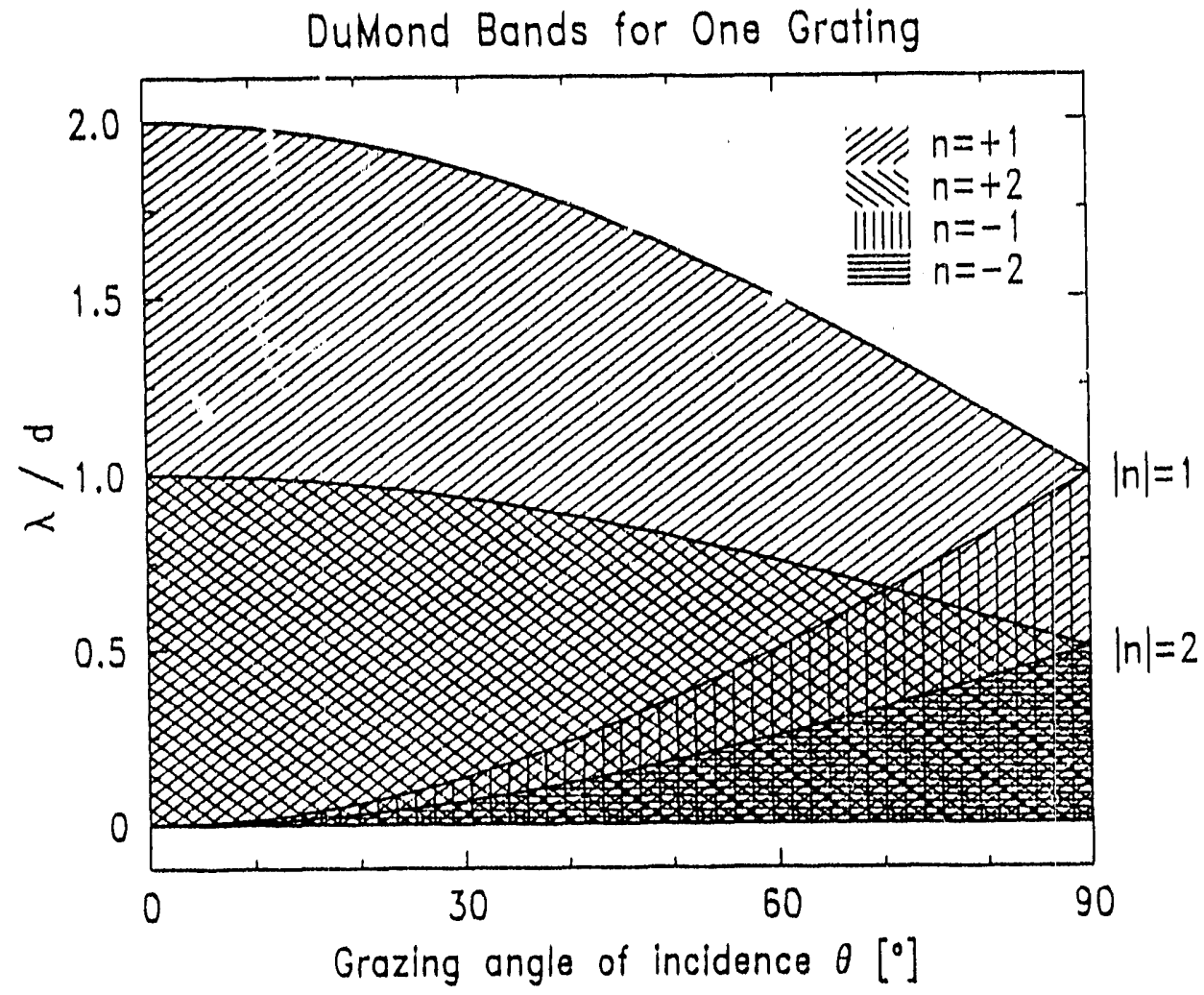

Fig. 27 The allowed ranges of wavelengths for first $(n=1)$ and second $(n=2)$ diffracted order from a diffraction grating, for both inside $(n>0)$ and outside $(n<0)$ orders, plotted as a function of the grazing angle of the incident ray.

The above discussion and the content of Fig. 27 are not intended to make any statements about the efficiency of diffraction by a grating other than to indicate the phase space in which it is nonzero. In fact, the grating diffiaction efficiency is a function of the angle of incidence $\alpha$, photon wavelength $\lambda$, groove spacing $d$, groove depth $h$, and land-to-groove ratio. A general discussion of grating diffraction efficiency for various optical geometries is provided by Franks, et al. [12]. A rigorous dynamical diffraction theory for reflection gratings has been developed [13], which exists in a commercially available computer program. 


\subsection{Phase Space in Geometric Optics}

The phase space transformation matrices for a plane grating illuminated by a small (in spatial and angular extent) bundle of incident rays are $\left[\begin{array}{cc}\frac{-\cos \beta}{\cos \alpha} & 0 \\ 0 & \frac{-\cos \alpha}{\cos \beta}\end{array}\right]$ for $\left(y, y^{\prime}\right)$ falling within one of the shaded regions in Fig. 27, and $\left[\begin{array}{ll}0 & 0 \\ 0 & 0\end{array}\right]$ otherwise.

These transformation matrices describe the small changes in position $(\mathrm{y})$ and angle $\left(y^{\prime}\right)$ of the small bundle of rays, relative to the principal ray, caused by the diffraction process. Note that the principal ray has changed in direction by $\alpha-\beta$, as determined by the grating equation (Eq. 16). The term relating $y^{\prime}$ emerging to $y^{\prime}$ in arises from expanding the grating equation for a small angular bundle of incident rays:

$$
\sin \left(\alpha+y^{\prime}{ }_{1 n}\right)=\sin \left(\beta+y^{\prime} \text { emorging }_{\text {in }}\right)=n \lambda / d
$$

For small $y_{\text {in }}^{\prime}$ and $y_{\text {emerging }}^{\prime}\left(\sin y^{\prime} \approx y^{\prime}, \cos y^{\prime} \approx 1\right)$, Eq. (17) becomes

$$
\sin \alpha+y^{\prime}{ }_{i n} \cos \alpha+\sin \beta+y^{\prime}{ }_{\text {emarging }} \cos \beta=n \lambda / d
$$

which, combined with the grating equation (Eq. 16) reduces to

$$
\frac{y^{\prime}{ }_{\text {emerging }}}{y^{\prime}{ }_{\text {in }}}=\frac{-\cos \alpha}{\cos \beta} \text {. }
$$

Note that the wavelength dependence of Eq. (19) is contained in the dependence of $\beta$ on $\lambda$, for a given $\alpha$, via the grating equation (Eq.16).

The phase space transformation matrices for a spherical grating illuminated by a small angular bundle of incident rays emanating from a point source (distance $\mathrm{p}$ from the grating) and focused to nearly a point image (distance $q$ from the grating)

in the Rowland circle steometry are: $\left[\begin{array}{cc}-\cos \beta / \cos \alpha & 0 \\ 1 / f=1 / p+1 / q & -\cos \alpha / \cos \beta\end{array}\right]$ for

$\left(y, y^{\prime}, \lambda\right)$ falling within one of the shaded regions in Fig. 27, and $\left[\begin{array}{ll}0 & 0 \\ 0 & 0\end{array}\right]$ otherwise [5]. In this diffraction geometry, the source, grating, and image lie on a circle of radius $\mathrm{R} / 2$, where $\mathrm{p}=\mathrm{R} \cos \alpha$ and $\mathrm{q}=\mathrm{R} \cdot \cos \beta$. 
Fig. 28 shows the optical layout of two popular soft x-ray nonochromators which utilize the Rowland circle geometry: the Grasshopper [14] and the Extended Range Grasshopper [15]. The Rowland circle grating diffraction geometry is similar to Fig. 25, except that the incident angle is $\alpha$ and is not generally equal to the diffracted angle $\beta$.

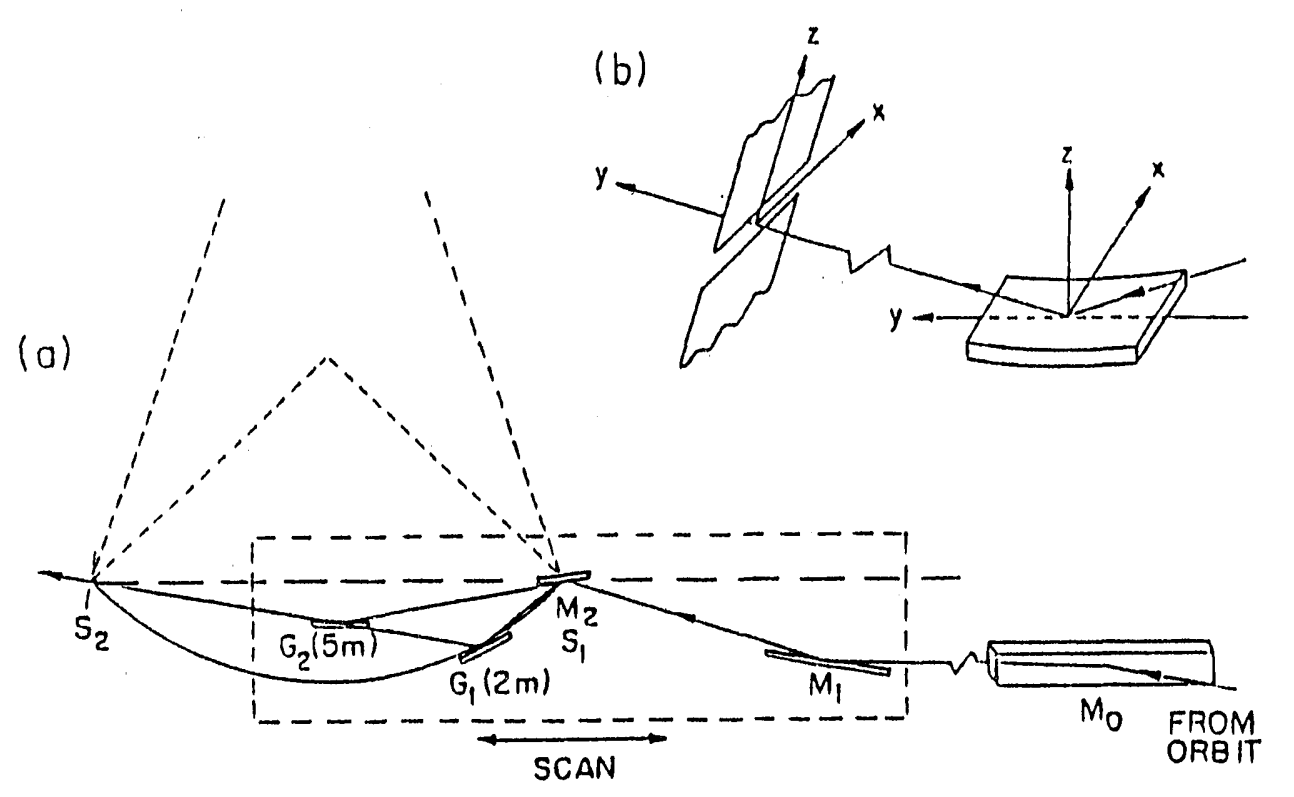

Fig. 28 Geometrical optical layout of the Extended Range Grasshopper (ERG) monochromator. Soft $\mathrm{x}$-rays from the source are focussed in the horizontal piane by the horizontal focusing mirror (M0) onto the sample (downstream of the exit slit S2) and in the vertical plane by the vertical focusing mirror (M1) onto the Codling mirror/entrance slit $(\mathrm{M} 2 / \mathrm{S} 1)$. The diffracted soft $\mathrm{x}$-rays from the grating (G1 or $\mathrm{G} 2$ ) are wavelength-selected by the exit slit S2. S1, G1 (G2), and S2 lie on a Rowland circle of $1 \mathrm{~m}(2.5 \mathrm{~m})$ radius. The position shown is zero order for the $5 \mathrm{~m}$ radius grating $\mathrm{G} 2$ and $160 \mathrm{eV}$ for a $2 \mathrm{~m}$ radius, 1200 groove $/ \mathrm{mm}$ grating $\mathrm{G} 1$. M1, M2/S1, G1, and G2 all translate together horizontally on an air bearing and M2/S1, G1, and $\mathrm{G} 2$ all rotate about the S1 slit axis in order to scan wavelength while maintaining fixed exit beam position and angle. The layout of the Grasshopper monochromator is identical to the one shown here, if the $5 \mathrm{~m}$ radius grating is omitted.

Thus, the phase space transformation matrix above may be written as:

$$
\left[\begin{array}{cc}
-\frac{q}{p} & 0 \\
\frac{1}{f}=\frac{1}{p}+\frac{1}{q} & -\frac{p}{q}
\end{array}\right] \text {. The terms relating } \mathrm{y}^{\prime} \text { emerging to } \mathrm{y}_{\text {in }} \text { ' and } \mathrm{y}_{\text {in }} \text { arise from }
$$


expanding the grating equation for a small angular bundle of incident rays on a curved grating surface in a form analogous to Eq. 17 above:

$$
\sin \left(\alpha+y^{\prime}{ }_{1 n}-y_{1 n} / p\right)+\sin \left(\beta+y^{\prime}{ }_{\text {omerglng }}+y_{\text {omerglng}} / q\right)=n \lambda / d .
$$

where $-y_{\text {in }} / p$ ( $y_{\text {emerging }} / q$ ) represent the variation in angle of incidence (emergence) for a ray parallel to but displaced by $y_{\text {in }}\left(y_{\text {emerging }}\right.$ ) from the principal ray.

For small $y^{\prime}{ }_{\text {in }}, y_{\text {in }} / p, y_{\text {emerging, }}^{\prime}$ and $y_{\text {emerging }} / q$, Eq. 20 leads to

$$
\left(y^{\prime} \text { in }-y_{\text {in }} / p\right) \cos \alpha+\left(y^{\prime} \text { emerging }+y_{\text {emerging }} / q\right) \cos \beta=0 \text {. }
$$

in an analogous way to Eq. 18 above. The relations $p=R \cos \alpha$ and $q=R \cos \beta$ allow Eq. 21 to be reduced to

$$
y^{\prime}{ }_{\text {in }} \cos \alpha-y_{\text {in }} / R+y_{\text {emerging }} \cos \beta+y_{\text {emerging }} / R=0 \text {. }
$$

Using $y_{\text {emerging }}=-q / p y_{\text {in }}$, we get

$$
y_{\text {omerging }}^{\prime}=\frac{-\cos \alpha}{\cos \beta} y_{i n}^{\prime}+\frac{1+\frac{g}{p}}{R \cos \beta} y_{i n}=-\frac{p}{q} y^{\prime}{ }_{i n}+\frac{1}{f} y_{i n} .
$$

It is instructive to evaluate the phase space transformation for the entire Rowland circle monochromator (entrance slit - grating - exit slit), as follows [5]:

$$
\left[\begin{array}{ll}
1 & q \\
0 & 1
\end{array}\right]\left[\begin{array}{cc}
-\frac{q}{p} & 0 \\
\frac{1}{f}=\frac{1}{p}+\frac{1}{q} & -\frac{p}{q}
\end{array}\right]\left[\begin{array}{ll}
1 & p \\
0 & 1
\end{array}\right]=\left[\begin{array}{cc}
1 & 0 \\
\frac{1}{f} & 1
\end{array}\right] \text { That is, for photons of a particular }
$$

wavelength, the Rowland circle monochromator has unity magnification and, for a point source, unity angular magnification. It should be noted that if a finite band of wavelengths is considered, the magnification in the Rowland geometry is not unity.

The M-K $\left(y, y^{\prime}, \lambda\right)$ matrices which describe the diffraction by a grating of a small bundle of rays centered around a principal ray must include $(y, \lambda)$ and $\left(y^{\prime}, \lambda\right)$ cross terms, and will not be presented here.

A popular alternative to the Rowland circle geometry is the constant-included-angle (CIA) geometry, in which the grating is merely rotated about its pole (no translations are required). A longtime workhorse in the VUV/soft x-ray energy range has been the toroidal grating monochromator (TGM) [16]. The toroidal profile provides both tangential and sagittal focusing, thereby reducing the number of reflections which must be made, i.e. increasing the transmission of the 
monochromator. However, the CIA geometry irtioduces significant aberrations the optical system, even for a point source. As summarized very usefully by Howells [17], a CIA monochromator with fixed entrance and exit armlengths suffers from significant defocus and coma aberrations. In addition, the TGM generally suffers from the astigmatic coma aberration. A significant advance in TGM design involved moving the exit slit to the wavelength-dependent best-focus position and choosing the focal properties of the entrance optics in a way that minimizes the sagittal filling of the grating (thereby minimizing astigmatic coma) [18].

In a ground breaking next step, the separated focusing Kirkpatrick-Baez entrance optics and the spherical grating of the Grasshopper design (see Fig. 28) were joined with the simplicity of the moving-exit-slit CIA design in a new monochromator design dubbed the Cylindrical Element Monochromator (CEM) or Spherical Grating Monochromator (SGM) [19]. The SGM suffers only from coma aberration (to third order), which is small compared to the photon energy spread caused by the surface figure error of the best spherical gratings produced to date. An optical layout of the SGM design is shown in Fig. 29.

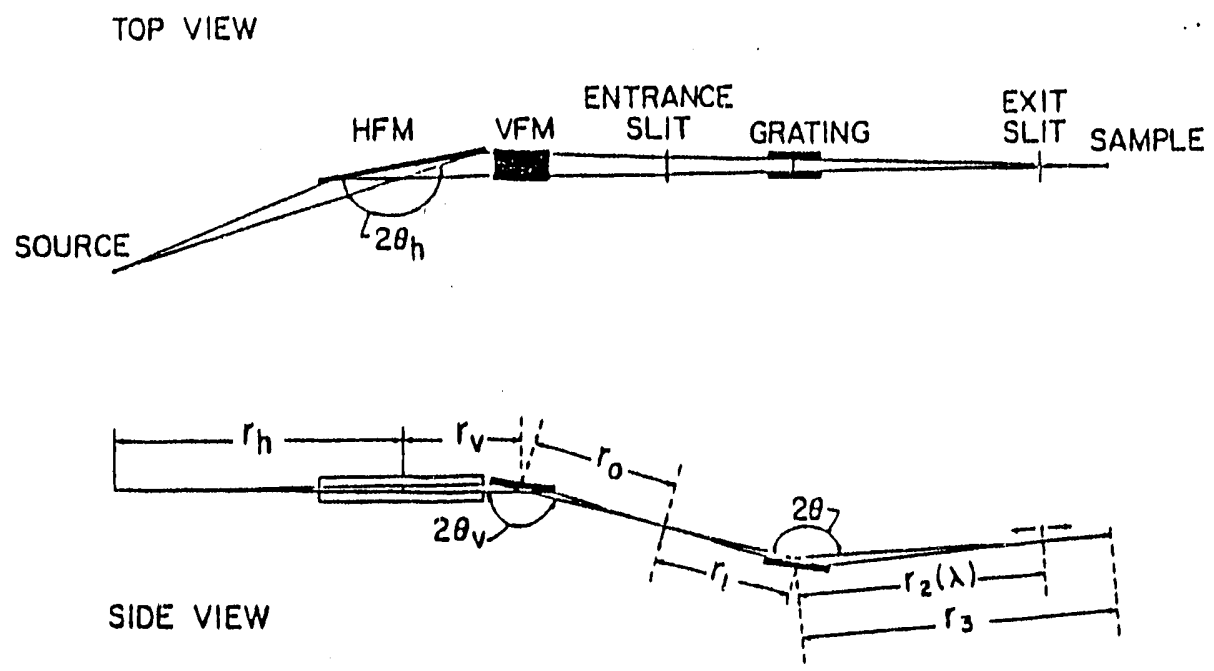

Fig. 29 Geometrical optical layout of the Spherical Grating Monochromator (SGM) monochromator. Soft $x$-rays from the source are focussed in the horizontal plane by the horizontal focusing mirror (HFM) onto the sample and in the vertical plane by the vertical focusing mirror (VFM) onto the entrance slit. The diffracted soft $x$-rays from the grating are wavelength-selected by the exit slit, which moves parallel to the principal ray to the wavelength-dependent best-focus position. 
A series of $M-K\left(y, y^{\prime}, \lambda\right)$ plots in the vertical plane for each of the optical elcuncuit: of the SGM monochromator aie presented in Fig. 30. The M-K plots for the Grasshopper and ERG monochromators are qualitatively identical to those of the SGM, since they differ mainly in the entrance and exit armlengths (the SGM armlengths are much longer). Fig. 30 is discussed in detail in section 4.3 below.
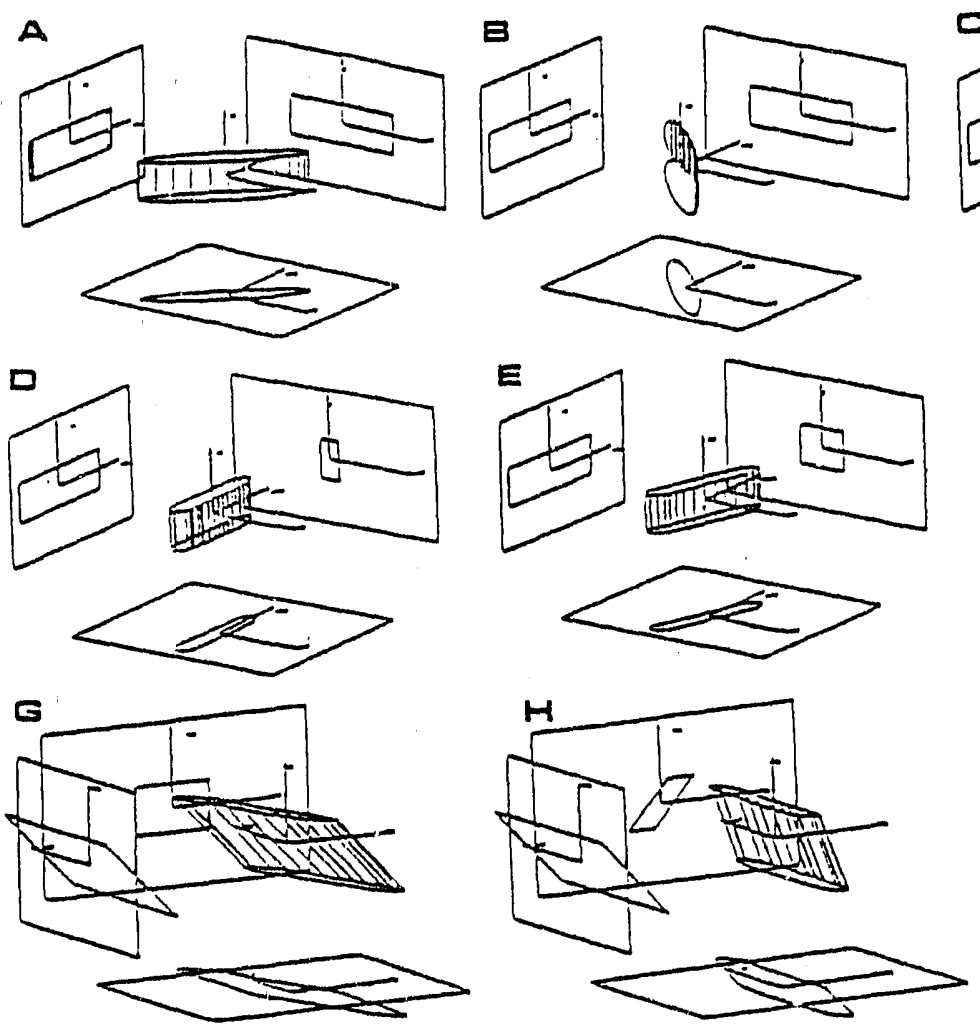
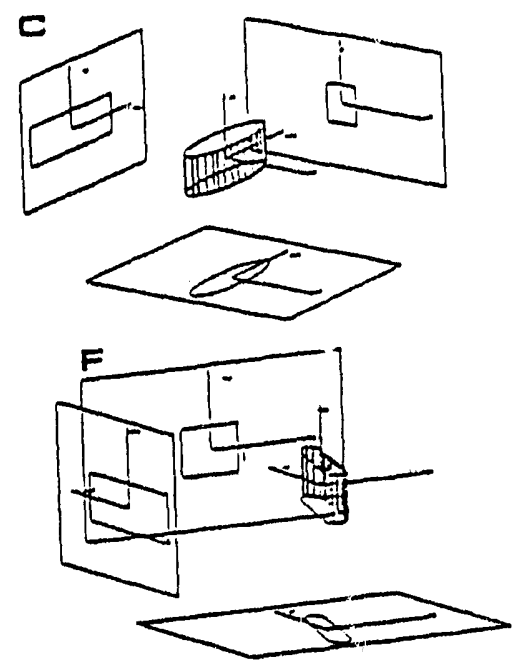

Fig. 30 A series of $M-K\left(y, y^{\prime}, \lambda\right)$ phase space diagrams in the vertical plane for each of the optical elements of the Spherical Grating Monochromator (SGM). In order, as the photons see them, the M-K diagrams represent (a) input to the vertical focusing mirror (VFM), (b) exit from VFM, (c) input to the entrance slit, (d) exit from the entrance slit, (e,f) input to the grating, (g) output from the grating, and $(\mathrm{h})$ the exit slit. For clarity of presentation, diagrams (a) through (e) are viewed from the $\left(+y,-y^{\prime},+\lambda\right)$ octant, and diagrams (f) through (h) are viewed from the viewed from the $\left(-y,-y^{\prime},+\lambda\right)$ octant. The M-K plots for the Grasshopper and ERG monochromators are qualitatively identical to those of the SGM, since the optical designs of the two monochromators differ mainly in the entrance and exit armiengths (the SGM armlengths are much longer). 
A completely different approach to VUV/soft $\mathrm{x}$-ray grating monochromalor's is to use a plane grating, rather than cylindrical, spherical, or toroidal. Generally, this leads to the use of aspheric mirrors to either prepare the beam prior to the grating and to focus it after the grating. A popular approach using plane gratings is the SX700 [20] monochromator, which allows the diverging rays from the source to diffract from the plarie grating in such a way that the rays exiting the grating appear to emerge from a virtual source which is many times farther from the grating than the real source. A large ellipsoidal mirror which focuses onto the exit slit is typically the most expensive optical element in the SX700 monochromator. Another approach, dubbed the Plane Grating Monochromator (PGM) [21], uses the plane grating as a nonfocusing dispersive element, i.e. the input rays must form a parallel bundle and the exit rays at each wavelength form a parallel bundle. The input rays are collimated using a paraboloid (P5), which treats the source as a distant point. Similarly, the exit rays are focussed onto the exit slit using another paraboloid (P1, P2, P3, or P4). An optical layout of the PGM monochromator is shown in Fig. 31.

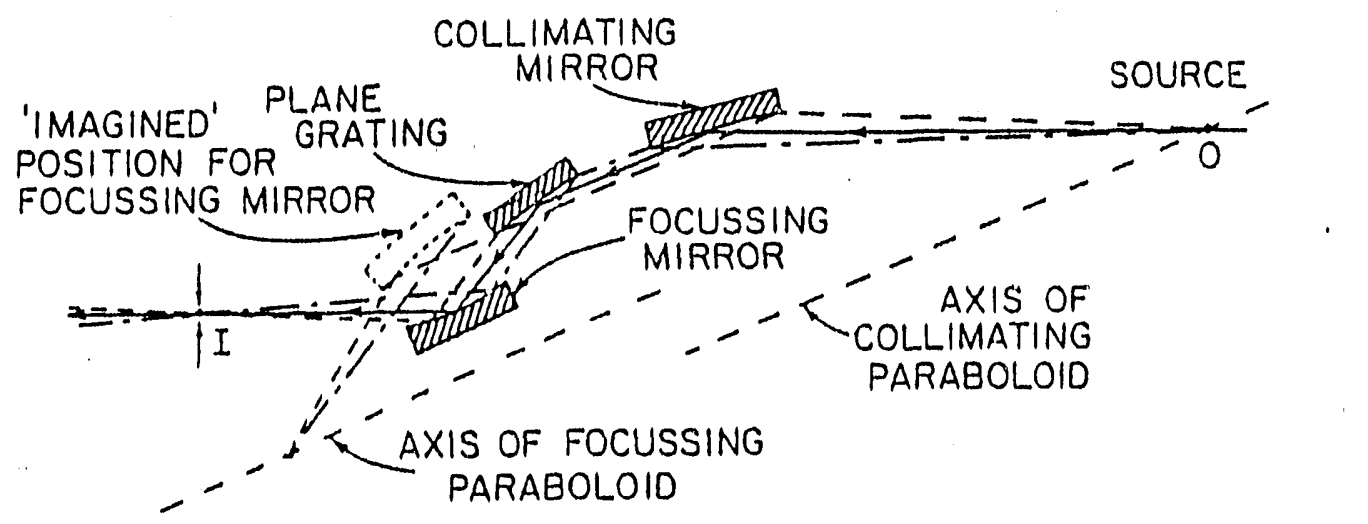

Fig. 31 Geometrical optical layout of the Plane Grating Monochromator (PGM) monochromator. The crosshatched optics are the normal configuration. Soft $\mathrm{x}$-rays from the source are rendered parallel by the parabolic collimating mirror (P5), diffracted by the grating and (still parallel) focused onto the exit slit by the parabolic focusing mirror (P1-4). The "imagined" position for P1-4 (dashed line) would be correct if the grating were removed from the optical system.

A series of $M-K\left(y, y^{\prime}, \lambda\right)$ plots in the vertical plane for each of the optical elements of the PGM monochromator are presented in Fig. 32 and discussed in detail in section 4.2 below. In terms of $\mathrm{M}-\mathrm{K}$ space description, the major difference between the PGM and SGM optical designs is in the shape of the $\left(y, y^{\prime}, \lambda\right)$ space volume incident on the grating. 

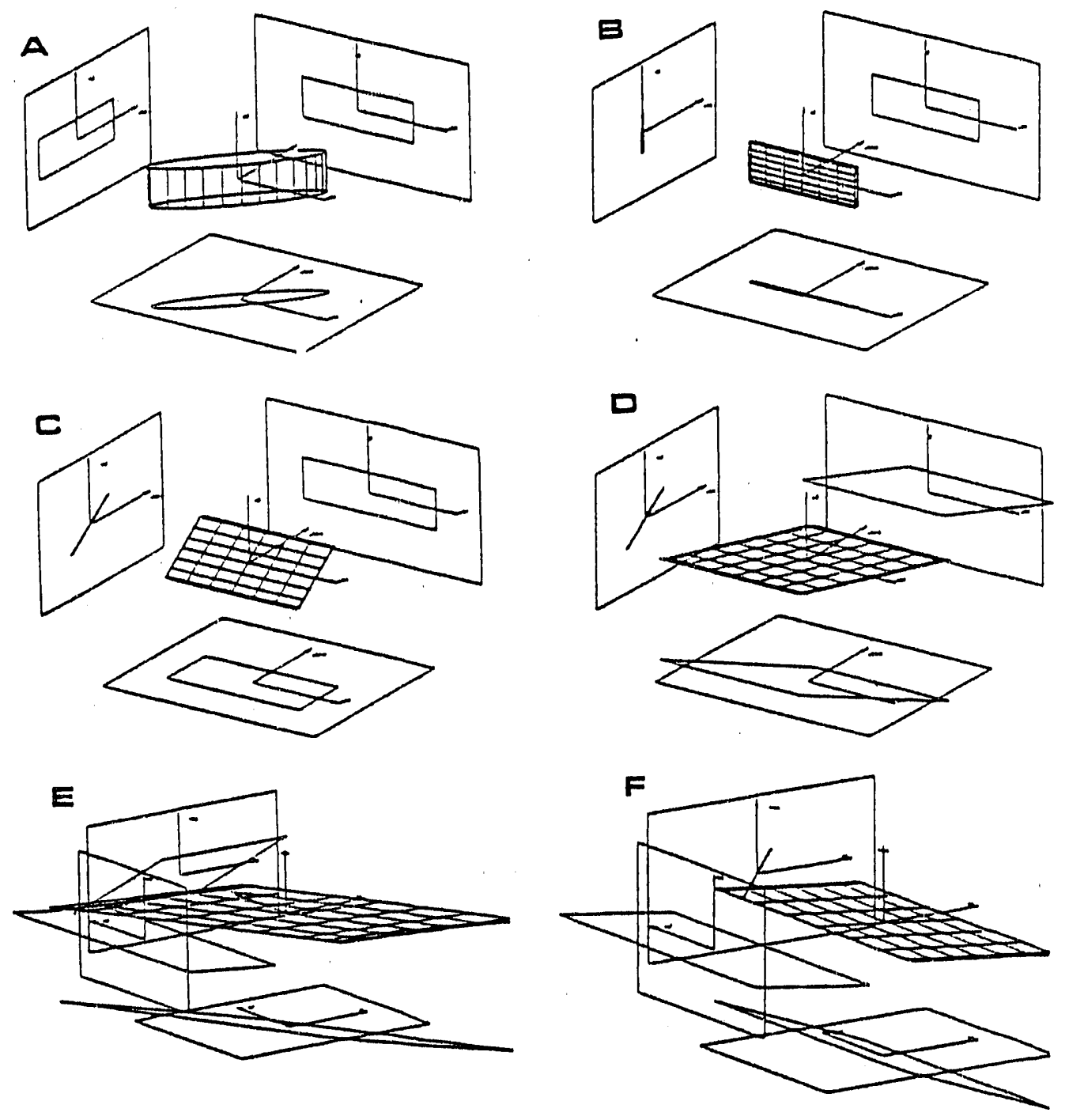

Fig. 32 A series of $M-K\left(y, y^{\prime}, \lambda\right)$ phase space diagrams in the vertical plane for each of the optical elements of the PGM monochromator. In order, as the photons see them, the M-K diagrams represent (a) input to the collimating paraboloid (P5), (b) exit from P5 (which is identical to the input to the grating), (c) output from he grating, (d) input to the refocusing paraboloid (P1-4), (e) output from P1-4, and (f) the exit slit. For clarity of presentation, diagrams (a) through (d) are viewed from the $\left(+y,-y^{\prime},+\lambda\right)$ octant, and diagrams (e) and (f) are viewed from the viewed from the $\left(-y,-y^{\prime},+\lambda\right)$ octant. 


\subsection{Plane Grating Monochromator}

A series of $M-K\left(y, y^{\prime}, \lambda\right)$ phase space diagrams in the vertical plane for each of the optical elements of the PGM optical design, presented in Fig. 32, demonstrate the dispersion and focusing properties of this monochromator. A series of $\mathrm{M}-\mathrm{K}$ $\left(y, y^{\prime}, \lambda\right)$ phase space diagrams in the vertical plane for each of the optical elements of the PGM monochromator. In order, as the photons see them, the M-K diagrams in Fig. 32 represent (a) input to the collimating paraboloid (P5), (b) exit from P5 (which is identical to the input to the grating), (c) output from he grating, (d) input to the refocusing paraboloid (P1-4), (e) output from P1-4, and (f) the exit slit. For clarity of presentation, diagrams (a) through (d) are viewed from the $\left(+y,-y^{\prime},+\lambda\right)$ octant, and diagrams (e) and ( $f$ ) are viewed from the viewed from the $\left(-y,-y^{\prime},+\lambda\right)$ octant. These $\mathrm{M}-\mathrm{K}$ diagrams are now discussed in more detail.

The SR source, drifted to the position of the P5 collimating mirror, is represented in $M-K$ space $\left(y, y^{\prime}, \lambda\right)$ by the upright elliptical cylinder shown in Fig. 32 (a). The P5 paraboloid collimates the rays, i.e. the y' range of the rays leaving P5 is negligibly small and the $\mathrm{M}-\mathrm{K}$ space volume representing the rays which emerge from P5 appears in Fig. 32(b) as a thin rectangular slab in the $(y, \lambda)$ plane.

This phase space does not change in drifting to the grating, since drifting depends only on the y' values which in this case are approximately zero. The grating dispersion is represented in M-K phase space as a rotation and stretching in the $\left(y^{\prime}, \lambda\right)$ plane, as given by the phase space transformation matrix for a plane grating above (for these graphical purposes the small y and $y^{\prime}$ dependence on $\lambda \dagger$ for all rays other than the principal ray is neglected).

Fig. 32(c) represents the output from the plane grating. Notice that there is no $y^{\prime}$ emerging dependence on $y_{\text {in }}$ in this case because all the incoming rays are parallel and intersect the plane grating surface at the same angle. However, the range of $y_{\text {emerging }}$ is magnified by $-\cos \beta / \cos \alpha$ and the range of $y^{\prime}$ emerging is accordingly demagnified by the same factor, as determined by the grating equation (Eq.16). Thus, the emerging rays from the grating are dispersed over a large range of angles [large slope in the $\left(y^{\prime}, \lambda\right)$ plane] but the $y^{\prime}$ extent for each wavelength is negligible, i.e. the rays at a given wavelength are still parallel.

In drifting from the grating to the refocusing paraboloid P1-4, the rays of

$t:$ recall that $y, y^{\prime}$, and $\lambda$ are the deviations in these quantities, relative to those of the principal ray, for each ray in the small bundle we are considering here. The spread in $y, y^{\prime}$, and $\lambda$ is taken to be small. 
different $y^{\prime}$ (which are also different in $\lambda$ ) spread apart in $y$, i.e. the tilted rectangular solid which represents the rays emerging from the grating skews to the right in $\left(y, y^{\prime}\right)$ phase space. The M-K space diagramof the input to P1-4 is shown in Fig. 32(d). The P1-4 refocusing mirror rotates this rectangular solid to the left of upright in $\left(y, y^{\prime}\right)$ phase space, which represents the fact that the emerging rays from $\mathrm{P} 1-4$, shown in Fig. 32(e) are drifting toward a focus.

The drift to the exit slit rotates the rays back to upright in the $\left(y, y^{\prime}\right)$ plane (i.e. $y$ focus), while preserving the $(y, \lambda)$ dependence generated by the grating dispersion. The M-K space diagram at the exit slit is shown in Fig 32(f). Thus, the exit slit, represented by a thin slab in the ( $\left.y^{\prime} \lambda\right)$ plane centered about $y=0$, transmits only a small band of wavelengths, i.e. wavelength selection has occurred. The rays which emerge from the exit slit have a relatively large spread in angle (y'), i.e. they diverge as they emerge from the exit slit. Often, another mirror is used after the exit slit in order to refocus the $x$-rays into a small spot, which is useful for many types of spectroscopy experiments.

The transmission of the PGM is the product of the M-K transformation matrices of the SR source, P5 collimating mirror, grating, P1-4 refocusing mirror, and exit slit. The product of these matrices will not be written down analytically (it is rather complicated and not too illuminating). Usually, ray tracing routines are used to evaluate the resolution and transmission of the PGM installed in a particular beamline on a particular storage ring. It is hoped that Fig. 32 gives the reader a physically intuitive picture of the function and $\mathrm{M}-\mathrm{K}$ space transformation of each optical element.

\subsection{Spherical Grating Monochromators}

The optical arrangement of the Grasshopper, ERG, TGM, and SGM monochromators are similar in the vertical plane: all of them focus onto an entrance slit and use a curved grating to disperse and focus onto an exit slit. For the purposes of the following discussion, we can refer to all of these monochromator designs as Spherical Grating Monochromators (SGMs) since they all utilize circular grating curvature in the tangential (dispersion) plane.

A series of $M-K\left(y, y^{\prime}, \lambda\right)$ phase space diagrams in the vertical plane for each of the optical elements of the SGM optical design, presented in Fig. 30, demonstrate the dispersion and focusing properties of this monochromator. In order, as the photons see them, the M-K diagrams in Fig. 30 represent (a) input to the collimating paraboloid (P5), (b) exit from P5 (which is identical to the input to the grating), (c) output from the grating, (d) input to the refocusing parat oloid (P1-4), (e) output from $P 1-4$, and ( $f$ ) the exit slit. For clarity of presentation, diagrams (a) through (d) are viewed from the $\left(+y,-y^{\prime},+\lambda\right)$ octant, and diagrams $(e)$ and $(f)$ are viewed from the 
viewed from the $\left(-y,-y^{\prime},+\lambda\right)$ octant. The $M-K$ plots for the Grasshopper and lit) ( $j$ monochromators are qualitatively identical to those of the SGM, since they differ mainly in the entrance and exit armlengths (the SGM armlengths are much longer). These $\mathrm{M}-\mathrm{K}$ diagrams are now discussed in more detail.

The SR source, drifted to the position of the vertical focusing mirror M1, is representer in $M-K$ space $\left(y, y^{\prime}, \lambda\right)$ by the upright elliptical cylinder shown in Fig. 30(a). M1 focuses the rays, i.e. the ( $\left.y, y^{\prime}\right)$ phase space ellipse of the rays leaving M1, Fig. 30(b), is rotated to the left of upright, indicating that the rays will drift toward a focus. The drift to the entrance slit (S0) rotates the rays back to upright in the $\left(y, y^{\prime}\right)$ plane, i.e. they come to a focus, the $M-K$ space diagram of the input to S0 is shown in Fig 30(c)and of the output from SO in Fig. 30(d).

The function of SO is to transmit a small, spatially weli-defined fraction of the rays incident upon it as a source for the dispersive optical element, i.e. the grating. The grating dispersion is represented in $\mathrm{M}-\mathrm{K}$ phase space as a rotation and stretching in the $(y, \lambda)$ plane, as determined by the phase space transformation matrix for a spherical grating discussed in section 4.1 above, and the focusing property of the curved grating is represented as a rotation in the $\left(y, y^{\prime}\right)$ plane (for these graphical purposes the small $y$ and $y$ ' dependence on $\lambda$ for all rays other than the principal ray is neglected). The $\mathrm{M}-\mathrm{K}$ space diagram of the input to the grating is show in Figs.30(e) and (f), and of the output from the grating in Fig. $30(\mathrm{~g})$. Note that, as for the PGM case in section 4.2 above, the range of $y_{\text {emerging }}$ is magnified by $-\cos \beta / \cos \alpha$ and the range of $y^{\prime}$ emerging is accordingly demagnified by the same factor, as determined by the grating equation (Eq. 16). The $y^{\prime}$ emerging values of the bundle of rays emerging from the grating differ from $y_{\text {in }}^{\prime}$ by $y_{\text {in }} / f$, where $y_{\text {in }}$ is the separation in the direction (vertical) of a given ray from the principal ray and $f$ is the focal length of the grating. This describes the focusing property of the curved grating: in the Rowland circle case (Grasshopper, ERG), $1 / f=1 / p+1 / q$; for the TGM and constant-included-angle SGM designs, the focal length is wavelength dependent.

As shown in Fig. $30(\mathrm{~g})$, the rays emerging from the grating are dispersed over a large range of angles [large slope in the $\left(y^{\prime}, \lambda\right)$ plane] and the rays of a particular wavelength are rotated to the left of upright in $\left(y, y^{\prime}\right)$ phase space, i.e. they will drift toward a wavelength dependent focus. In drifting from the grating to the exit slit S1, the rays of different $y^{\prime}$ (which are also different in $\lambda$ ) spread apart in $y$, i.e. the tilted elliptical $M-K$ space solid which represents the rays emerging from the grating skews to the right in $\left(y, y^{\prime}\right)$ phase space. At the same time, the drift to the exit slit rotates the rays back to upright in the $\left(y, y^{\prime}\right)$ plane, while preserving the $(y, \lambda)$ dependence generated by the grating dispersion. The $M-K$ space diagram of the exit slit $\mathrm{S} 1$ is shown in Fig.30(h), the exit slit transmits only a small band of wavelengths, i.e. wavelength selection has occurred by spatial filtering of the dispersed rays in the 
exit slit plane. The rays which emerge from the exit slit have a relatively lin:" divergence, as for the PGM case above. Often, another mirror is used after lic: (x) slit in order to refocus the $x$-rays into a small spot, which is useful for many types of spectroscopy experiments.

The transmission of the SGM is the product of the M-K transformation matrices of the SR source, M0 horizontal focusing mirror, M1 vertical focusing mirror, entrance slit SO, grating, and exit slit. The product of these matrices will not be written down analytically (it is rather complicated and not too illuminating). Usually, ray tracing routines are used to evaluate the resolution and transmission of a particular SGM installed in a particular beamline on a particular storage ring. Rather, it is hoped that Fig. 30 gives the reader a physically intuitive picture of the function and $\mathrm{M}-\mathrm{K}$ space transformation of each optical element.

\subsection{Sensitivity to Storage Ring Parameters}

The performance of the monochromators described above depends crucially on the value and stability of many of the SR storage ring parameters. The most important storage ring parameters in terms of their effect on bearnline monochromator performance are the beam size, beam position stability, opening angle, angle stability, and stored current. It should be noted that various experiments place further requirements on the storage ring parameters, e.g. on the time structure and stability of the SR pulses.

\subsubsection{Current}

The requirement on stored current usually is that it be as large and stable as possible. The only exception to this occurs if the heating of optical elements or slits in the beamline at high beam currents changes the performance of the beamline in an unwanted or time dependent way.

\subsubsection{Vertical beam size and position}

The vertical beam size affects the PGM and SGM monochromators in completely different ways. In the PGM case, larger beam size results in a larger source size contribution to the transmitted bandwidth because this design uses no entrance slit. That is, the energy resolution of the monochromator is directly related to the vertical beam size. The maximum acceptable beam size is determined by the energy resolution requirement of the experiment being performed. Within the time scale of one spectrum, vertical beam motion is equivalent to increased vertical beam size. Thus, vertical beam motion on time scales less than that required to acquire one spectrum also decreases the average energy resolution of the PGM for that spectrum. If this energy resolution degradation can be tolerated, however, the large 
vertical acceptance of the PGM design makes its transmission stable against veiluk beam movement.

The use of an entrance slit in the SGM monochromators guarantees that the photon wavelength and resolution are stable against changes in vertical beam size or vertical beam position. This wavelength stability is achieved at the expense of transmission: the throughput of the entrance slit, and hence of the entire beamline, is directly affected by changes in either vertical beam size or position. The usual remedy for this problem is to implement a monitor of the flux output from the monochromator, which is then used to normalize the experimental data. The use of a flux monitor is crucial, because most soft $x$-ray SGM beamline designs attempt to maximize throughput by exactly matching the demagnified source to the entrance slit. Therefore, any increase in beam size or changes in beam position usually are observed iminediziely as changes in transmission.

\subsubsection{Horizontal beam size and position}

The SGM monochromator design almost completely decouples its vertical and horizontal functions. Its optical elements are all large radius spheres and are oriented in either the vertical or horizontal planes. Thus, horizontal beam size and position changes are merely transmitted to the exit slit with some magnification or demagnification, depending on the choice of horizontal focusing mirror. The PGM uses paraboloidal mirrors before and after the grating, which couple horizontal and vertical motions. Therefore, the energy resolution of the PGM monochromator is in principle affected by horizontal beam size and position changis. However, this horizontal/vertical coupling is insignificant for small (less than a few $\sigma$ ) horizontal beam size changes or motions.

\subsubsection{Vertical beam opening angle and angular position}

Both the PGM and SGM monochromators are much more tolerant of changes in vertical beam opening angle than of changes in vertical beam size. For ideal optical element surfaces, a larger source vertical opening angle merely increases the filling of these optics and does not change the transmission of the monochromator as long as none of the optics is overfilled. Of course, the vertical opening angle of the $x$-rays emerging from the exit slit will increase as the source vertical opening angle is increased.

As discussed above for beam size considerations, most soft $x$-ray beamlines attempt to maximize throughput by exactly matching the magnified source angular opening to the angular acceptance of the grating. In order to demonstrate this point, Figs. 33 and 34 compare the vertical opening angle of two NSLS VUV ring SR sources with the angular acceptance of some NSLS VUV soft $x$-ray beamlines as a 
function of photon energy, Fig. 33 compares the NSLS VUV SR vertical opening angle, magnified by the first bearnline mirror, with the vertical opening angle accepted by the gratings of the PGM and ERG monochromators. Note that the vertical angular acceptance of each ERG grating approximately matches the magnified source opening angle at the high energy end of its operating range, but that the PGM gratings are all significantly overfilled by the VUV source output. Fig. 34 compares the NSLS U13U wiggler SR vertical opening angle, magnified by the first U13UA beamline mirror (M1), with the vertical opening angle accepted by the gratings of the SGM monochromator. The five gratings for the new high resolution SGM monochromator at NSLS U13UA are overfilled for up to half of their photon energy ranges. This is the result of a tradeoff involving demagnification by M1 in order to maximize the transmission of the entrance slit (SO).

Vert. opening angles: NSLS VUV source output; ERG, PGM acceptance

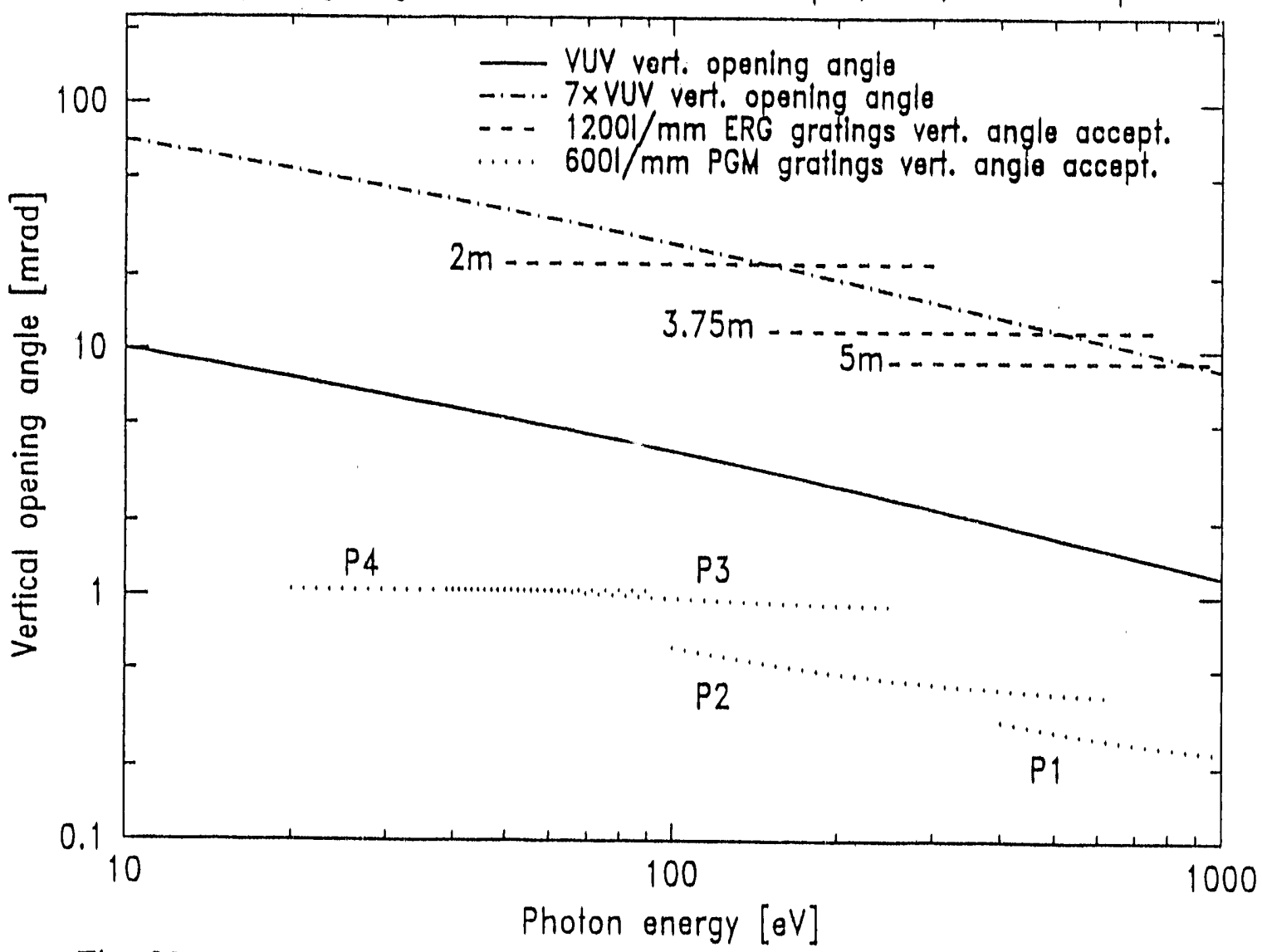

Fig. 33 The NSLS VUV SR vertical opening angle, m. nnified by the first beamline mirror, and the vertical opening angle accepted by the zratings of the PGM and ERG monochromators, as a function of photon energy. Note that the vertical angular acceptance of each ERG grating approximately matches the magnified source opening angle at the high energy end of its operating range, but that the PGM gratings are all significantly overfilled by the VUV source output. 
Vertical opening angles: UT3UA source output and SGM accerinita

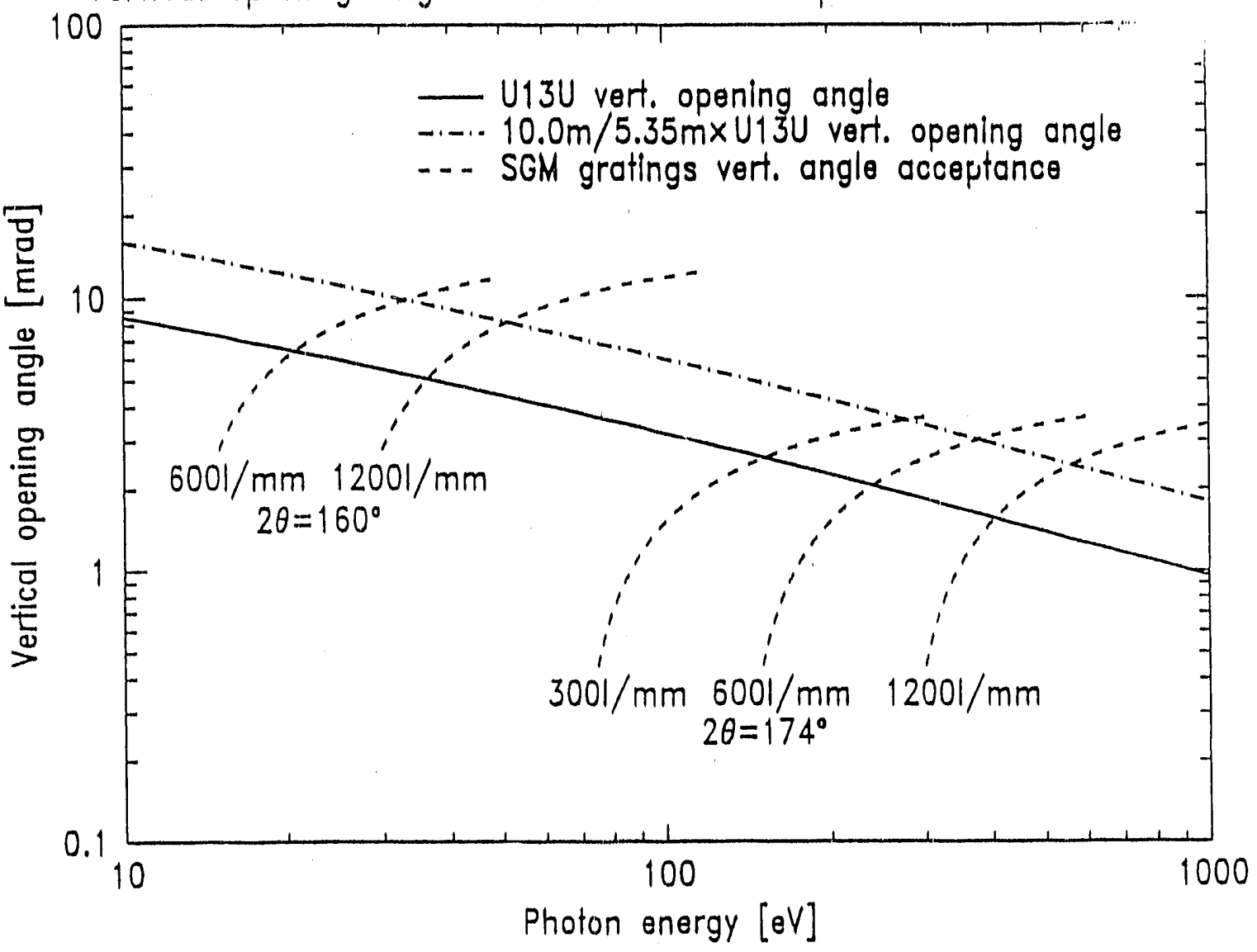

Fig. 34 The NSLS U13U wiggler SR vertical opening angle, magnified by the first U13UA beamline mirror (M1), and the vertical opening angle accepted by the gratings of the SGM monochromator, as a function of photon energy. The five gratings for the new high resolution SGM monochromator at NSLS U13UA are overfilled for up to half of their photon energy ranges. This is the result of a tradeoff involving demagnification by $\mathrm{M} 1$ in order to maximize the transmission of the entrance slit (SO).

\subsubsection{Horizontal beam opening angle and angular postion}

Since bending magnet SR is uniform in the horizontal plane, the optical elements of monochromators which use this radiation (e.g. PGM, ERG, TGM, SGM) are always fully illuminated horizontally. Therefore, they are completely insensitive to changes in horizontal beam opening angle. The U13UA SGM monochromator has been designed to accept the full horizontal opening angle of the U13U wiggler radiation at maximum $\mathrm{K}$. Owing to the decoupled horizontal and vertical functions of the SGM optics, horizontal beam opening angle or angle position changes merely alter the horizontal position and intensity distribution at the exit slit, but do not affect the vertical properties of the monochromator. 


\subsection{Concluding Remarks}

In the previous sections we have shown how some typical optical systems can be represented in phase space, and by now, the reader should appreciate that variations in the source phase space will affect the phase space of the optical system in predictable ways. For example, beamlines which have dispersive crystal optics without focussing can be quite insensitive to beam motion with regard to selected wavelength, although beam translations at the source will appear at the sample.

For some experiments such as bulk transmission extended $x$-ray absorption fine structure (EXAFS) beam motion of this kind may be acceptable, while for experiments with very small samples such as protein crystallography where samples are often as small as 100 microns, beam motion of $1 \mathrm{~mm}$ at the sample would render the measurement completely useless. In geometric optical systems which focus to entrance and/or exit slits, such motions will translate either to changes in included angle for systems without an entrance slit (the grating sees the source directly), which shifts the transmitted wavelength, or to changes in intensity on the sample for systems with an entrance slit (the included angle is fixed by the location of slits and grating). Variations in intensity are for the experimenter generally only an annoyance in as much as incident flux monitors are usually incorporated into the experiment. Wavelength shifts are however more difficult to deal with because it is often not possible to monitor them directly, so beam motion may in fact corrupt data which are sensitive to wavelength, which includes nearly every experiment performed at a storage ring. This problem has at many facilities been the driving force behind ambitious programs to enhance photon beam stability.

We hope that this contribution will serve as an introduction to the behavior of optical systems with respect to the source and its phase space, and further, that it will provide the accelerator physics community with some common ground with the synchrotron radiation user community for understanding the link between the users and the machine.

*Work performed under the auspices of the U.S. Department of Energy, under contract DE-AC02-76CH00016. 


\subsection{REFERENCES}

1. For example see:

(Overview) R. Reininger, Proceedings of 1989 CERN Accelerator School on Synchrotron Radiation and Free Electron Lasers, CERN 90-03, p401. (Crystal Optics)T. Matsushita and H. Hashizume, X-Ray Monochromators in: Handbook of Synchrotion Radiation, Vol 1, ed E.E. Koch (North Holland, Amsterdam, 1983) Ch4.

R. Caciuffo, S. Melone, F. Rustichelli, and A. Boeuf, Phus. Reports 152(1987) 1 .

(Geometric Optics) V. Saile; and J.B. West, Nucl. Instr. and Meth. 208 (1983) 199.

R.L. Johnson Nucl. Ir .. and Meth. A246 (1986) 303.

J.B. West and H.A. Padmore, Optical Engineering, in Handbook of Synchrotron Radiation, Vol. 2, ed G.V. Marr (Elsevier Science Publishers, Amsterdam, 1987) Ch 2.

2. J. Wong, G. Shimkaveg, W. Goldstein, M. Eckart, T. Tanaka, Z. Rek, H. Tompkins, Nucl. Inst. and Methods, A291(1990)243

3. L.E. Berman, CHESS Technical Memorandum Number 24 (1981).

4. J.B. Hastings, J. Appl. Phys 48(1977)1576.

5. T. Matsushita and U. Kaminaga, J. Appl. Cryst. 13 [I](1980)464

6. T. Matsushita and U. Kaminaga, J. Appl. Cryst. 13 [1](1980)472.

7. J.W.M. DuMond, Physical Review 52(1937)872-83.

8. G.K. Green (1976), Brookhaven National Lab Report \# 50522.

9. J.M. Weber and S.L. Hulbert, BNL informal report xxx (1991)

10. A.H. Compton, and S.K. Allison, (1957), X-Rays in Theory and Experiment, Princeton: D.Van Nostrand.

11. D.P. Siddons, J.B. Hastings, and G. Faigel, Nucl. Instrum. Methods, A266(1988)329.

12. A. Franks, K. Lindsay, J.M. Bennett, R.J. Speer, D. Tuner, and D.J. Hunt, Phil. Trans. Roy. Soc. 277,503 (1975). 
13. W. Jark and M. Neviere, Appl. Opt. 26(1987)943, and references theres..

14. F. C. Brown, R. Z. Bachrach, and N. Lien, Nucl. Instrum. Methods 152(1978)73.

15 S. L. Hulbert, J. P. Stott, F. C. Brown, and N. C. Lien, Nucl. Instrum. Methods 208(1983)43, and F. C. Brown, J. P. Stott, and S. L. Hulbert, Nucl. Instrum. Methods 246(1986)278.

16 P. Thiry, C. Depautex, R. Pinchaux, Y. Petroff, D. Lepe're, and A. Passereau, Nucl. Instrum. Methods 172(1980)172;

B. P. Tonner, Nucl Instrum. Methods 172(1980)133;

and F. J. Himpsel, Y. Jugnet, D. E. Eastman, J. J. Donelon,D. Grimm, G. Landgren, A. Marx, J. F. Morar, C. Oden, R. A. Pollak, J. Schneir, and C. A. Crider, Nucl. Instrum. Methods 222(1984) 107.

17 M. R. Howells, in X-Ray Data Booklet, ed. D. Vaughan, LBL PUB-490 (1986); geometrical theory of diffraction gratings can be found in H. Noda, T. Namioka, and M. Seya, J. Opt. Soc. Am. 64(1974)1031.

18 C. T. Chen, E. W. Plummer, and M. R. Howells, Nucl. Instrum. Methods 222(1984)103.

19 C. T. Chen, F. Sette, Nucl. Instrum. Meth. A256(1987)59:5.

20 H. Petersen, Nucl. Instrum. Meth. A246(1986)260;

H. Petersen, BESSY Technical Bulletin 136 (1988);

and, F. C. Brown and S. L. Hulbert, Nucl. Instrum. Methods 222(1984)42.

21. N. R. Howells, Nucl. Instrum. Methods 177(1980)127;

and G. P. Williams, M. R. Howells, N. Lucas, and P. Z. Takacs, Nucl. Instrum. Methods 222(1984)99. 
